\title{
ON THE ABSENCE OF LARGE-ORDER DIVERGENCES IN SUPERSTRING THEORY
}

\author{
Simon Davis \\ Institut für Mathematik \\ Universität Potsdam \\ D-14415 Potsdam, Germany
}

\begin{abstract}
The genus-dependence of multi-loop superstring amplitudes is estimated at large orders in perturbation theory using the super-Schottky group parameterization of supermoduli space. Restriction of the integration region to a subset of supermoduli space and a single fundamental domain of the super-modular group suggests an exponential dependence on the genus. Upper bounds for these estimates are obtained for arbitrary N-point superstring scattering amplitudes and are shown to be consistent with exact results obtained for special type II string amplitudes for orbifold or Calabi-Yau compactifications. The genus-dependence is then obtained by considering the effect of the remaining contribution to the superstring amplitudes after the coefficients of the formally divergent parts of the integrals vanish as a result of a sum over spin structures. The introduction of supersymmetry therefore leads to the elimination of large-order divergences in string perturbation theory, a result which is based only on the supersymmetric generalization of the Polyakov measure and not the gauge group of the string model.
\end{abstract}

PACS Code: 11.25.Db 


\section{Introduction}

Calculations of scattering amplitudes in type II superstring theory reveal that the S-matrix is free from the infrared and ultraviolet divergences arising in the quantum amplitudes derived from the linearized Ricci action. A third type of divergence, occurring in both field theories and bosonic string theories, is produced by the growth of terms at large orders in the perturbation series. It will be shown that large-order divergences are absent from superstring perturbation expansion, providing confirmation of the consistency of the theory at the quantum level.

The elimination of the third type of infinity can be traced to the introduction of supersymmetry and simultaneous occurrence of infrared and large-order divergences in the infinite-genus limit. A connection has been established between the genus-dependence of the limits for the Schottky group parameters and the large-order behaviour of the bosonic string amplitudes. ${ }^{1,2}$ The superstring measure is different, but the configurations of handles, the limits for the bosonic variables, multipliers and fixed points of the Schottky group, and the conditions for the fundamental domain of the symplectic modular group remain unchanged, so that this connection is still valid in superstring theory. Finiteness of superstring amplitudes at each order in the loop expansion has already been demonstrated, and since the large-order behaviour of superstring amplitudes is determined in part by the sum of the amplitudes with different degeneration limits, occurring either separately or simultaneously, it will be shown that N-point g-loop amplitudes do not grow at a factorial rate but give rise to a summable series which converges for sufficiently small values of the coupling constant. The absence of large-order divergences shall be demonstrated for the sum over finite-genus Riemann surfaces. While the possibility of including infinite-genus surfaces is briefly discussed in $\S 3$, the conclusions regarding the large-order divergences will not be extended to this category, but rather a result pertaining to the string coupling constant is obtained.

Another indication of the higher degree of finiteness of string amplitudes at large orders is the growth of the regularized closed bosonic string partition function. Restriction of the integration range in the Schottky parameterization to a single copy of the fundamental region of the modular group reduces the regularized path integral by a genus-dependent factor. 1 Although the regularized integral increases rapidly with respect to the genus, as a typical configuration of handles contributes $\frac{g^{g}}{(\ln g)^{13 g}}$, this is significantly less than the $g$ ! growth of scattering amplitudes in bosonic field theories. ${ }^{2}$ Moreover, large-order divergences in the bosonic string perturbation series, arising from regularization of tachyon infinities occurring at each order, are an artifact of the S-matrix expansion for this theory. Divergences of this type, for example, occur in theories containing renormalons, which often arise when there are infrared instabilities. The growth of the regularized closed bosonic string partition function, therefore, is not necessarily linked to observable instanton effects, suggesting that the introduction of supersymmetry may lead to the elimination of the large-order divergences in bosonic string theory. This result is supported by recent work on the appearance of an $N$-fold supersymmetry which appears in theories where the Borel singularity of the perturbation expansion vanishes for the first $N$ excited states ${ }^{3}$ and the bounding of correlation functions in fermionic 
field theories. ${ }^{4}$

Whereas in field theories, instantons are reflected in the perturbation series through a factorial increase in the terms, as the Borel transform has a singularity in the coupling constant plane, it has been shown that non-perturbative effects in string theories are described by a separate sum over surfaces with Dirichlet boundaries inserted, ${ }^{5}$ so that the inclusion of these boundaries and higher-dimensional soliton configurations in the sum over histories would be sufficient to obtain a complete formulation of the quantum theory. The geometry of these nonperturbative configurations also is suggestive of the connection between different superstring theories through duality. Estimates of finite-genus amplitudes and conclusions regarding the viability of superstring perturbation theory then would remain valid within this framework.

\section{The Superstring Measure and the Fundamental Domain}

Superstring scattering amplitudes are given by the supermoduli space integrals ${ }^{6}$

$$
\begin{aligned}
\left\langle V_{1}\left(k_{1}\right) \ldots V_{N}\left(k_{N}\right)\right\rangle_{g}=\int_{s \mathcal{M}_{g}} d \mu_{s W P}\left(\frac{8 \pi s \operatorname{det}^{\prime} \hat{\square}_{0}}{\operatorname{sdet}\left\langle\hat{\Psi_{\alpha}} \mid \hat{\Psi}_{\beta}\right\rangle}\right)^{-5} & \left(\operatorname{sdet} \hat{\mathcal{P}}_{1}^{\dagger} \hat{\mathcal{P}}_{1}\right)^{\frac{1}{2}} \\
& \left\langle\left\langle V_{1}\left(k_{1}\right) \ldots V_{N}\left(k_{N}\right)\right\rangle\right\rangle_{\hat{E}}
\end{aligned}
$$

where $d \mu_{s W P}$ is the super Weil-Petersson measure, $\Psi_{\alpha} \in K e r \square_{0},\langle\langle\rangle\rangle$ represents the evaluation of the path integral over the scalar position superfields $X^{\mu}$, and the integral is restricted to a $(3 g-3 \mid 2 g-2)$ complex-dimensional slice of super-Teichmuller space parametrized by the supergeometries $\left\{\hat{E}_{M}^{A}\right\}$. In terms of superghosts,

$$
\begin{gathered}
\left\langle V_{1}\left(k_{1}\right) \ldots V_{N}\left(k_{N}\right)\right\rangle_{g}=\int_{s \mathcal{M}_{g}} d^{2} m_{K} \int D(X B C)\left\langle\left\langle V_{1}\left(k_{1}\right) \ldots V_{N}\left(k_{N}\right)\right\rangle\right\rangle \prod_{b}\left|\delta\left(\left\langle\mu_{b} \mid B\right\rangle\right)\right|^{2} \\
\prod_{k}\left|\left\langle\mu_{k} \mid B\right\rangle\right|^{2} e^{-I}
\end{gathered}
$$

where $\mathrm{B}$ and $\mathrm{C}$ are anti-ghost and ghost superfields of $\mathrm{U}(1)$ weight $\frac{3}{2}$ and -1 respectively, $\mu_{K}, K=(k, b)$, are super Beltrami differentials, and $I=I_{m}+I_{s g h}$ is the sum of the matter and superghost actions. ${ }^{6}$ A similar formula for the scattering amplitude of $\mathrm{N}$ external massless states appears in the twistor-string formalism. ${ }^{7}$

The Schottky uniformization of super-Riemann surfaces shall be used to study the superstring measure. The super-Schottky group is generated by $g$ transformations $T_{n}, n=1, \ldots, g$, acting on the super-complex plane with coordinate $Z=(z, \theta)$

$$
\frac{T_{n}(Z)-Z_{1 n}}{T_{n}(Z)-Z_{2 n}}=K_{n} \frac{Z-Z_{1 n}}{Z-Z_{2 n}} \quad\left|K_{n}\right|<1
$$

where $Z_{1 n}=\left(\xi_{1 n}, \theta_{1 n}\right)$ and $Z_{2 n}=\left(\xi_{2 n}, \theta_{2 n}\right)$ are attractive and repulsive super-fixed points respectively. 
It has been found that the measure on supermoduli space for the Neveu-Schwarz sector of superstring theory, corresponding to the propagation of bosonic states in the loops, is simpler than that of the Ramond sector. ${ }^{8-10}$ The holomorphic part multiplied by the period-matrix factor is

$$
\begin{gathered}
\frac{1}{d \mathcal{V}_{A B C}} \prod_{n=1}^{g} \frac{d K_{n}}{K_{n}^{\frac{3}{2}}} \frac{d Z_{1 n} d Z_{2 n}}{Z_{1 n}-Z_{2 n}}\left(\frac{1-K_{n}}{1-(-1)^{B_{n}} K_{n}^{\frac{1}{2}}}\right)^{2}[\operatorname{det}(\operatorname{Im} \mathcal{T})]^{-5} \\
\cdot \prod_{\alpha} \prod_{p=1}^{\infty}\left(\frac{1-(-1)^{N_{\alpha}^{B}} K_{\alpha}^{p-\frac{1}{2}}}{1-K_{\alpha}^{p}}\right)^{10} \prod_{\alpha}^{\prime} \prod_{p=2}^{\infty}\left(\frac{1-K_{\alpha}^{p}}{1-(-1)^{N_{\alpha}^{B}} K_{\alpha}^{p-\frac{1}{2}}}\right)^{2}
\end{gathered}
$$

where the infinitesimal super-projective invariant volume element is

$$
\begin{aligned}
d \mathcal{V}_{A B C} & =\frac{d Z_{A} d Z_{B} d Z_{C}}{\left[\left(Z_{A}-Z_{B}\right)\left(Z_{C}-Z_{A}\right)\left(Z_{B}-Z_{C}\right)\right]^{\frac{1}{2}}} \cdot \frac{1}{d \Theta_{A B C}} \\
\Theta_{A B C} & =\frac{\theta_{A}\left(Z_{B}-Z_{C}\right)+\theta_{B}\left(Z_{C}-Z_{A}\right)+\theta_{C}\left(Z_{A}-Z_{B}\right)+\theta_{A} \theta_{B} \theta_{C}}{\left[\left(Z_{A}-Z_{B}\right)\left(Z_{C}-Z_{A}\right)\left(Z_{B}-Z_{C}\right)\right]^{\frac{1}{2}}}
\end{aligned}
$$

and the super-period matrix is

$$
\mathcal{T}_{m n}=\frac{1}{2 \pi i}\left[\ln K_{n} \delta_{m n}+\sum_{\alpha}{ }^{(m, n)} \ln \left[\frac{Z_{1 m}-V_{\alpha} Z_{1 n}}{Z_{1 m}-V_{\alpha} Z_{2 n}} \frac{Z_{2 m}-V_{\alpha} Z_{2 n}}{Z_{2 m}-V_{\alpha} Z_{1 n}}\right]\right]
$$

Selecting $B_{n}$ to be 0 or 1 , depending on the boundary conditions around the $g$ B-cycles, produces $2^{g}$ spin structures associated with the exchange of bosonic states in the $g$ loops. 11-13 The number $N_{\alpha}^{B}$ equals $\sum_{n=1}^{g} B_{n} N_{\alpha}^{n}$, where $N_{\alpha}^{n}$ is the number of times that the generator $T_{n}$ or its inverse appear in the product $V_{\alpha}$. Since the choice of spin structure is defined by the sign of the square root $K_{n}^{\frac{1}{2}}$ in equation (2.4), a modular transformation maps the expression with a given choice of signs for the square roots $K_{n}^{\frac{1}{2}}$ for all $n$ into a measure with different signs for the square roots, corresponding to another spin structure, altering the integral by factors determined by the genus-dependence of primitive-element products.

The overall $O S p(2 \mid 1)$ invariance can be used to fix two of the super-fixed points and the even coordinate of a third superfixed point, leaving $3 g-3$ even moduli and $2 g-2$ odd moduli amongst the super-Schottky group parameters. ${ }^{8}$ The integration region is defined to be the fundamental domain of the super-mapping class group in super-Teichmuller space. Equivalently, one may use the intersection of the fundamental region of the super-modular group in the space of positive-definite, symmetric super-period matrices with the set of $\mathcal{T}_{m n}$ associated with a super-Riemann surface, which will be contained in the set of $\mathcal{T}_{m n}$ such that the ordinary period matrix $\tau_{m n}$, the complex number-valued part of $\mathcal{T}_{m n}$, lies in a fundamental domain of the modular group and corresponds to a Riemann surface. These constraints on $\tau_{m n}$ lead to an infinite number of conditions on the multipliers $K_{n}$ and fixed points $\xi_{1 n}, \xi_{2 n},{ }^{1,2}$, which can be reduced to a finite number at large genus. 
In the analysis of the closed bosonic string, these inequalities may be satisfied by certain categories of isometric circles, $I_{T_{n}}=\left\{z \in \hat{\mathbb{C}}|| \gamma_{n} z+\delta_{n} \mid=1\right\}, T_{n} z=\frac{\alpha_{n} z+\beta_{n}}{\gamma_{n} z+\delta_{n}}$, which would then represent a subset of moduli space. In particular, the following configurations of isometric circles
(i) $\frac{\epsilon_{0}}{g^{1-2 q^{\prime}}} \leq\left|K_{n}\right| \leq \frac{\epsilon_{0}^{\prime}}{g^{1-2 q^{\prime}}}$
$\delta_{0} \leq\left|\xi_{1 n}-\xi_{2 n}\right| \leq \delta_{0}^{\prime}$
$0 \leq q^{\prime} \leq \frac{1}{2}$
(ii) $\frac{\epsilon_{0}}{g^{1-2 q^{\prime}}} \leq\left|K_{n}\right| \leq \frac{\epsilon_{0}^{\prime}}{g^{1-2 q^{\prime}}}$
$\frac{\delta_{0}}{g^{q}} \leq\left|\xi_{1 n}-\xi_{2 n}\right| \leq \frac{\delta_{0}^{\prime}}{g^{q}}$
$0<q \leq q^{\prime}<\frac{1}{2}$
(iii)

$$
\epsilon_{0} \leq\left|K_{n}\right| \leq \epsilon_{0}^{\prime}
$$$$
\frac{\delta_{0}}{\sqrt{g}} \leq\left|\xi_{1 n}-\xi_{2 n}\right| \leq \frac{\delta_{0}^{\prime}}{\sqrt{g}}
$$

describe a subset of Teichmuller space consistent with a cut-off on the radii of the isometric circles, or equivalently the size of the handles in the intrinsic metric on the surface, $r_{I_{T_{n}}}^{2}=$ $\left|\gamma_{n}\right|^{-2} \gtrsim \frac{1}{g} .14$

While the parameters $q$ and $q^{\prime}$ are initially chosen to be continuous, to avoid overcounting in the path integral, a discrete set of values must be chosen. Non-overlapping of the ranges $\frac{\epsilon_{0}}{g^{1-2 q^{\prime}}} \leq\left|K_{n}\right| \leq \frac{\epsilon_{0}^{\prime}}{g^{1-2 q^{\prime}}}$ requires that the following values of $q^{\prime}$ be selected

$$
q_{N}^{\prime}=N \frac{\ln \left(\frac{\epsilon_{0}^{\prime}}{\epsilon_{0}}\right)}{2 \operatorname{lng}} \quad N=0,1, \ldots,\left[\frac{\ln g}{\ln \left(\frac{\epsilon_{0}^{\prime}}{\epsilon_{0}}\right)}\right]
$$

The discrete value of $q$ follows from the bound

$$
\left|\gamma_{n}\right|^{-2}=\frac{\left|K_{n}\right|}{\left|1-K_{n}\right|^{2}}\left|\xi_{1 n}-\xi_{2 n}\right|^{2} \geq \frac{\epsilon_{0}}{g^{1-2 q^{\prime}}} \cdot \frac{\delta_{0}}{g^{2 q}} \cdot \frac{1}{\left|1-\frac{\epsilon_{0}^{\prime}}{g^{1-2 q^{\prime}}}\right|^{2}} \gtrsim \mathcal{O}\left(\frac{1}{g}\right)
$$

While the cut-off on the length of the closed geodesics is not modular invariant, it is the genus-independence of the cut-off which is necessary for an estimate of regularized bosonic string partition function. An $S L(2, \mathbb{C})$-invariant regulator exists for the superstring, ${ }^{15}$ but the finiteness of the superstring amplitudes implies that any upper bound can be chosen independently of the cut-off, which may be removed to obtain the total amplitude.

The measure for the other Neveu-Schwarz-Ramond sectors shall given in an abbreviated form in $\S 3$. The total amplitude in the super-Schottky parametrization, obtained by summing over all spin structures, is invariant under super-modular transformations. ${ }^{16}$

Earlier studies of primitive-element products show that they can be bounded by exponential functions of the genus. ${ }^{2,14}$ Bounds similar to the those obtained for bosonic strings can be set for the primitive-element products in the measure for the Neveu-Schwarz sector. 


$$
\begin{gathered}
\left|\prod_{n=1}^{g}\left(\frac{1-K_{n}}{1-K_{n}^{\frac{1}{2}}}\right)^{2} \cdot \prod_{\alpha}^{\prime} \prod_{p=1}^{\infty}\left(\frac{1-K_{\alpha}^{p-\frac{1}{2}}}{1-K_{\alpha}^{p}}\right)^{10} \prod_{\alpha}^{\prime} \prod_{p=2}^{\infty}\left(\frac{1-K_{\alpha}^{p}}{1-K_{\alpha}^{p-\frac{1}{2}}}\right)^{2}\right|^{2} \\
=\prod_{n=1}^{g}\left|1-K_{n}\right|^{4} \cdot \prod_{n=1}^{g}\left|1-K_{n}^{\frac{1}{2}}\right|^{-4} \cdot \prod_{\alpha}^{\prime} \prod_{p=1}^{\infty}\left|1-K_{\alpha}^{p-\frac{1}{2}}\right|^{16} \\
\cdot \prod_{\alpha}^{\prime} \prod_{p=1}^{\infty}\left|1-K_{\alpha}{ }^{p}\right|^{-16} \cdot \prod_{\alpha}{ }^{\prime}\left|1+K_{\alpha}\right|^{-4}
\end{gathered}
$$

Upper bounds for each of the factors in the products can be readily be given

$$
\begin{aligned}
& \prod_{n=1}^{g}\left|1-K_{n}\right|<\prod_{n=1}^{g}\left(1+\left|K_{n}\right|\right) \leq \exp \left(\sum_{n=1}^{g}\left|K_{n}\right|\right) \\
& \prod_{n=1}^{g}\left|1-K_{n}^{\frac{1}{2}}\right| \leq \exp \left(\sum_{n=1}^{g}\left|K_{n}\right|^{\frac{1}{2}}\right) \\
& \prod_{\alpha} \prod_{p=1}^{\infty}\left|1-K_{\alpha}^{p-\frac{1}{2}}\right|<\prod_{\alpha}^{\prime}\left(1+\left|K_{\alpha}\right|^{\frac{1}{2}}\right)^{16} \cdot \prod_{\alpha}^{\prime} \prod_{p=2}^{\infty}\left(1+\left|K_{\alpha}\right|^{p-\frac{1}{2}}\right)^{16} \\
&<\prod_{\alpha}^{\prime}\left(1+\left|K_{\alpha}\right|^{\frac{1}{2}}\right)^{16} \cdot \prod_{\alpha}^{\prime} \prod_{p=1}^{\infty}\left(1+\left|K_{\alpha}\right|^{p}\right)^{16} \\
&<\exp \left(16 \sum_{\alpha}^{\prime}\left|K_{\alpha}\right|^{\frac{1}{2}}\right) \exp \left(16 \sum_{\alpha}^{\prime} \frac{\left|K_{\alpha}\right|}{1-\left|K_{\alpha}\right|}\right)
\end{aligned}
$$

Since

$$
\begin{gathered}
\frac{1}{1-\left|K_{\alpha}\right|}=1+\frac{1}{1-\left|K_{\alpha}\right|}\left|K_{\alpha}\right|<1+\frac{1}{1-\epsilon_{0}^{\prime}}\left|K_{\alpha}\right| \\
\prod_{\alpha}^{\prime} \prod_{p=1}^{\infty} \frac{1}{\left(1-\left|K_{\alpha}\right|^{p}\right)^{16}}<\exp \left(\frac{16}{1-\epsilon_{0}^{\prime}} \sum_{\alpha}^{\prime} \frac{\left|K_{\alpha}\right|}{1-\left|K_{\alpha}\right|}\right) \\
\prod_{\alpha}^{\prime} \frac{1}{\left(1-\left|K_{\alpha}\right|^{\frac{1}{2}}\right)^{4}}<\exp \left(\frac{4}{1-\epsilon_{0}^{\prime \frac{1}{2}}} \sum_{\alpha}^{\prime}\left|K_{\alpha}\right|^{\frac{1}{2}}\right)
\end{gathered}
$$

Combining equations (2.13)-(2.15) gives

$$
\begin{array}{r}
\left|\prod_{\alpha} \prod_{p=1}^{\infty}\left(\frac{1-K_{\alpha}^{p-\frac{1}{2}}}{1-K_{\alpha}^{p}}\right)^{8}\right|^{2}\left|\prod_{\alpha}^{\prime}\left(1+K_{\alpha}^{\frac{1}{2}}\right)^{-2}\right|^{2}<\exp \left(16 \cdot \frac{2-\epsilon_{0}^{\prime}}{1-\epsilon_{0}^{\prime}} \sum_{\alpha}^{\prime} \frac{\left|K_{\alpha}\right|}{1-\left|K_{\alpha}\right|}\right) \\
\cdot \exp \left(\frac{20-16 \epsilon_{0}^{\prime \frac{1}{2}}}{1-\epsilon_{0}^{\prime \frac{1}{2}}} \sum_{\alpha}^{\prime}\left|K_{\alpha}\right|^{\frac{1}{2}}\right)
\end{array}
$$


In categories (i) and (ii), $\frac{\epsilon_{0}}{g^{\frac{1}{2}-q^{\prime}}} \leq\left|K_{n}\right|^{\frac{1}{2}} \leq \frac{\epsilon_{0}^{\prime \frac{1}{2}}}{g^{\frac{1}{2}-q^{\prime}}}$, and $\epsilon_{0} g^{\frac{1}{2}+q^{\prime}} \leq \sum_{n=1}^{g}\left|K_{n}\right|^{\frac{1}{2}} \leq \epsilon_{0}^{\prime \frac{1}{2}} g^{\frac{1}{2}+q^{\prime}}$. Similarly, for isometric circles in the third category, $\epsilon_{0} g \leq \sum_{n=1}^{g}\left|K_{n}\right|^{\frac{1}{2}} \leq \epsilon_{0}^{\prime} g$, so that $\prod_{n=1}^{g}\left|1-K_{n}^{\frac{1}{2}}\right|^{-4} \leq \exp \left(-4 \epsilon_{0} g\right)$.

The sum $\sum_{\alpha}{ }^{\prime}\left|K_{\alpha}\right|$ has been shown to increase linearly with the genus, and the same property is valid for $\sum_{\alpha}{ }^{\prime} \frac{\left|K_{\alpha}\right|}{1-\left|K_{\alpha}\right|} .{ }^{1}$ Similar bounds cannot be placed on the sum $\sum_{\alpha}{ }^{\prime}\left|K_{\alpha}\right|^{\frac{1}{2}}$. Consider, for instance, isometric circles in category (i), with $q^{\prime}=0$, so that $\frac{\epsilon_{0}}{g} \leq\left|K_{n}\right| \leq \frac{\epsilon_{0}^{\prime}}{g}$ and $\delta_{0} \leq\left|\xi_{1 n}-\xi_{2 n}\right| \leq \delta_{0}^{\prime}$. Then,

$$
\left|\gamma_{n_{l}}\right|<\frac{\epsilon_{0}^{\prime \frac{1}{2}}}{1-\frac{\epsilon_{0}^{\prime}}{g}} \frac{\delta_{0}^{\prime}}{\sqrt{g}}
$$

and

$$
\sum_{n_{l}} \frac{\left|\gamma_{n_{l}}\right|^{-1}}{\left|\xi_{1 n_{l}}+\frac{\delta_{n_{l}}}{\gamma_{n_{l}}}\right|}+\sum_{n_{l}} \frac{\left|\gamma_{n_{l}}\right|^{-1}}{\left|\xi_{2 n_{l}}-\frac{\alpha_{n_{l}}}{\gamma_{n_{l}}}\right|}<\frac{\epsilon_{0}^{\prime \frac{1}{2}}}{1-\frac{\epsilon_{0}^{\prime}}{g}} \frac{\delta_{0}^{\prime}}{\delta_{0}} \cdot 2 \sqrt{g}
$$

Similarly, when $V_{\tilde{\alpha}}=T_{n_{l_{1}}} T_{n_{l_{2}}}$,

$$
\left|\gamma_{\tilde{\alpha}}\right|^{-1}=\left|\gamma_{n_{l_{1}}}\right|^{-1}\left|\gamma_{n_{l_{2}}}\right|^{-1}\left|\frac{\delta_{n_{l_{1}}}}{\gamma_{n_{l_{1}}}}+\frac{\alpha_{n_{l_{2}}}}{\gamma_{n_{l_{2}}}}\right|^{-1} \leq \frac{\epsilon_{0}^{\prime}}{\left(1-\frac{\epsilon_{0}^{\prime}}{g}\right)^{2}} \frac{\delta_{0}^{\prime 2}}{g}\left|\frac{\delta_{n_{l_{1}}}}{\gamma_{n_{l_{1}}}}+\frac{\alpha_{n_{l_{2}}}}{\gamma_{n_{l_{2}}}}\right|^{-1}
$$

Assuming that the average value in a dense packing of isometric circles about the circle $I_{T_{n_{l_{1}}, j_{1}}^{-1}}$ is proportional to $\sqrt{g},{ }^{1}$ it can be shown that the $r$ factors of $6\left[l^{T_{n_{l_{r}}}^{ \pm 1}}-1\right]$ produces a factor of order $\mathcal{O}\left(g^{r-1}\right)$ in $\sum_{\tilde{\alpha}}\left|K_{\tilde{\alpha}}\right|^{\frac{1}{2}}$ while the product $\left|\gamma_{n_{l_{1}}}\right|^{-1} \ldots\left|\gamma_{n_{l_{r}}}\right|^{-1} \rightarrow \mathcal{O}\left(\frac{1}{g^{\frac{\pi}{2}}}\right)$, so that the entire sum over elements consisting of $r$ generators grows as $\mathcal{O}\left(g^{\frac{r}{2}-1}\right)$.

This result can also be shown by considering the contribution of a particular region in parameter space, $\left\{K_{n}^{\frac{1}{2}} \mid \arg K_{n}^{\frac{1}{2}} \in[0, v]\right\}$, to the primitive-element products and super-moduli space integral will be estimated. The following proposition may be used:

Proposition. Consider two elements $\mathrm{S}$ and $\mathrm{T}$ of the Schottky group. If $\left|\xi_{1 S}+\frac{\delta_{S}}{\gamma_{S}}\right|=$ $\mathcal{O}\left(\frac{1}{g^{r}}\right),\left|\xi_{1 T}+\frac{\delta_{T}}{\gamma_{T}}\right|=\mathcal{O}\left(\frac{1}{g^{r^{\prime}}}\right)$, and $\left|-\frac{\delta_{T}}{\gamma_{T}}+\frac{\delta_{S}}{\gamma_{S}}\right|=\mathcal{O}\left(\frac{1}{g^{r^{\prime \prime}}}\right)$, then $K_{S T}=K_{S} K_{T}$ and $\arg \left(K_{S T}\right) \simeq \arg K_{S}+\arg K_{T}$ when $r^{\prime \prime}>\max \left(r, r^{\prime}\right)$ or $r^{\prime \prime}<\min \left(r, r^{\prime}\right)$. If $r^{\prime \prime}$ does equal either $r$ or $r^{\prime}$, then the argument $\arg K_{S T}$ can take values in a much wider range. 
Proof. The multiplier is

$$
K_{T}=T^{\prime}\left(\xi_{1 T}\right)=\frac{\gamma_{T}^{-2}}{\left(\xi_{1 T}+\frac{\delta_{T}}{\gamma_{T}}\right)^{2}}=\frac{\alpha_{T}+\delta_{T}-\sqrt{\left(\alpha_{T}+\delta_{T}\right)^{2}-4}}{\alpha_{T}+\delta_{T}+\sqrt{\left(\alpha_{T}+\delta_{T}\right)^{2}-4}}
$$

and

$$
\begin{aligned}
(S T)^{\prime}\left(\xi_{1}^{(S T)}\right) & =S^{\prime}\left(T\left(\xi_{1}(S T)\right) T^{\prime}\left(\xi^{S T}\right)=\frac{\gamma_{S}^{-2}}{\left(T\left(\xi_{1}^{(S T)}\right)+\frac{\delta_{S}}{\gamma_{S}}\right)^{2}} \frac{\gamma_{T}^{-2}}{\left(\xi_{1}^{(S T)}+\frac{\delta_{T}}{\gamma_{T}}\right)}\right. \\
K_{S T} & =K_{S} K_{T}\left|\frac{\xi_{1 S}+\frac{\delta_{S}}{\gamma_{S}}}{T\left(\xi_{1}^{(S T)}\right)+\frac{\delta_{S}}{\gamma_{S}}} \frac{\xi_{1 T}+\frac{\delta_{T}}{\gamma_{T}}}{\xi_{1}^{(S T)}+\frac{\delta_{T}}{\gamma_{T}}}\right|^{2} \\
& \simeq K_{S} K_{T}\left|\frac{\xi_{1 S}+\frac{\delta_{S}}{\gamma_{S}}}{\xi_{1 T}+\frac{\delta_{S}}{\gamma_{S}}} \frac{\xi_{1 T}+\frac{\delta_{T}}{\gamma_{T}}}{\xi_{1 S}+\frac{\delta_{T}}{\gamma_{T}}}\right|^{2}
\end{aligned}
$$

Since $\xi_{1}^{(S T)}$ and $T\left(\xi_{1}^{(S T)}\right)$ is located near $\xi_{1 S}$ and $\xi_{1 T}$ respectively,

$$
\begin{aligned}
\arg \left[\frac{(S T)^{\prime}\left(\xi_{1}^{(S T)}\right)}{S^{\prime}\left(\xi_{1 S}\right) T^{\prime}\left(\xi_{1 T}\right)}\right] & \approx \arg \left[\left(\xi_{1 S}+\frac{\delta_{S}}{\gamma_{S}}\right)^{2}\left(\xi_{1 T}+\frac{\delta_{T}}{\gamma_{T}}\right)^{2}\right] \\
& -\arg \left[\left(\xi_{1 T}+\frac{\delta_{S}}{\gamma_{S}}\right)^{2}\right]-\arg \left[\left(\xi_{1 S}+\frac{\delta_{T}}{\gamma_{T}}\right)^{2}\right]
\end{aligned}
$$

Consider the two pairs of isometric circles $\left\{I_{S}, I_{S^{-1}}\right\}$ and $\left\{I_{T}, I_{T^{-1}}\right\}$. Let $\arg \left(\xi_{1 S}+\frac{\delta_{S}}{\gamma_{S}}\right)=$ $-\alpha_{S}, \arg \left(\xi_{1 T}+\frac{\delta_{T}}{\gamma_{T}}\right)=\alpha_{T}, \arg \left(\xi_{1 T}+\frac{\delta_{S}}{\gamma_{S}}\right)=-\theta_{T S}$, $\arg \left(\xi_{1 S}+\frac{\delta_{T}}{\gamma_{T}}\right)=\theta_{S T}$. Then $\chi_{1}=\theta_{S T}-\alpha_{T}, \chi_{2}=\theta_{T S}-\alpha_{S}$ and

$$
\arg K_{S T}-\arg K_{S}-\arg K_{T} \approx-2 \alpha_{S}+2 \alpha_{T}-2 \theta_{S T}+2 \theta_{T S}=2\left(\chi_{2}-\chi_{1}\right)
$$

When $\left|\xi_{1 S}+\frac{\delta_{S}}{\gamma_{S}}\right|,\left|\xi_{1 T}+\frac{\delta_{T}}{\gamma_{T}}\right| \ll\left|-\frac{\delta_{T}}{\gamma_{T}}+\frac{\delta_{S}}{\gamma_{S}}\right|, \chi_{1}, \chi_{2} \simeq \frac{\pi}{2}$, and similarly, if $\left|\xi_{1 S}+\frac{\delta_{S}}{\gamma_{S}}\right|, \mid \xi_{1 T}+$ $\frac{\delta_{T}}{\delta_{S}}|\gg|-\frac{\delta_{T}}{\gamma_{T}}+\frac{\delta_{S}}{\gamma_{S}} \mid, \chi_{1}, \chi_{2} \simeq 0$. For both configurations, the cross-ratio in the ratio of $K_{S T}$ to $K_{S} K_{T}$ has approximately unit magnitude and $\chi_{2}-\chi_{1} \simeq 0$, so that $\arg \left(K_{S T}\right) \approx$ $\arg K_{S}+\arg K_{T}$.

Given an even distribution of the arguments of $K_{n}^{\frac{1}{2}}$ in the interval [0,v], the argument of multiplier $K_{\alpha}^{\frac{1}{2}}$ of the Schottky group element $V_{\alpha}=T_{n_{1}} \ldots T_{n_{l}}$ would equal approximately $\sum_{j=1}^{l} \arg K_{n_{j}}^{\frac{1}{2}}$, a property which can be used in obtaining a bound for the product

$$
\prod_{\alpha}^{\prime}\left|1+K_{\alpha}^{\frac{1}{2}}\right|=\prod_{l=1}^{\infty} \prod_{\alpha_{(l)}}\left|1+K_{\alpha}^{\frac{1}{2}}\right|
$$


Suppose that the angles $\arg K_{n}^{\frac{1}{2}}, n=1, \ldots, g$ take the values $\frac{v}{g}, \ldots, v$ in any particular order. Following the convention that the element $T_{n}^{-1}$ has reversed fixed points $\xi_{2 n}, \xi_{1 n}$ and the same multiplier $K_{n}$, it follows that the each argument for the set $\left\{T_{n}^{ \pm 1}, n=1, \ldots, g\right\}$ occurs twice in the sequence $\frac{v}{g}, \ldots, v$. Adding these arguments $l$ times produces a distribution of angles $\left\{\arg K_{\alpha(l)}\right\}$ determined by the composition function, $c(N, l, M)$, which represents the number of ordered partitions of the integer $M$ into $l$ parts with each addend less than or equal to $N$, multiplied by an overall factor of $2^{l}$ because of the two different elements having multipliers with the same arguments. ${ }^{17}$

The number of compositions of $M$ into $l$ parts is

$$
\begin{aligned}
c(l, M)= & \left(\begin{array}{c}
M-1 \\
l-1
\end{array}\right)=\frac{(M-1) !}{(l-1) !(M-l) !} \\
c(N, l, M)= & \sum_{k=0}^{\left.\frac{(M+l)}{N}\right]}(-1)^{k}\left(\begin{array}{l}
l \\
k
\end{array}\right)\left(\begin{array}{c}
l-1+M-k N \\
l-1
\end{array}\right)
\end{aligned}
$$

where the maximum value of $M$ is $l_{N}$ and the mean value is $\bar{M}_{l}=\left[\frac{l(N+1)}{2}\right]$, where the peak of the distribution $c(N, l, M)$ for $1 \leq M \leq M_{\max }$. occurs, since $c(N, l, M)=c(N, l, l(N+1)-M)$.

The following asymptotic formula for the number of compositions is valid when $M=\frac{1}{2}\left(l-k^{\prime}\right)(N+1), 0 \leq k^{\prime} \leq K l^{\beta}$ with $0 \leq \beta<\frac{1}{2}$ and some constant $K .{ }^{18}$

$$
\begin{aligned}
c(N, l, M)= & \frac{1}{\sqrt{\pi}}\left(\frac{6}{N^{2}-1}\right)^{\frac{1}{2}} \frac{N^{l}}{l^{\frac{1}{2}}} \\
& {\left[1+\frac{h_{1,0}+h_{1,1} k^{\prime 2}}{l}+\ldots+\frac{\sum_{j=0}^{m=1} h_{m-1, k}(N) k^{\prime 2 j}}{l^{m-1}}+O\left(\frac{1+k^{\prime 2 m}}{l^{m}}\right)\right] } \\
h_{1,0}= & -\frac{3\left(N^{2}+1\right)}{20\left(N^{2}-1\right)} \quad h_{1,1}=-\frac{3(N+1)}{2(N-1)} \\
h_{2,0}= & -\frac{13 N^{2}-134 N^{2}+13}{1120\left(N^{2}-1\right)^{2}} \quad h_{2,1}=\frac{9\left(N^{2}+1\right)}{8(N-1)^{2}} \\
h_{2,2}= & \frac{9(N+1)^{2}}{8(N-1)^{2}}
\end{aligned}
$$

The peak value is reached at the mean $\bar{M}$ when $k^{\prime}=0$ 


$$
\begin{aligned}
c(N, l, \bar{M}) & =\frac{1}{\sqrt{\pi}}\left(\frac{6}{N^{2}-1}\right)^{\frac{1}{2}} \frac{N^{l}}{l^{\frac{1}{2}}}\left[1+\frac{h_{1,0}}{l}+\frac{h_{2,0}}{l^{2}}+\ldots\right] \\
& \simeq \frac{1}{\sqrt{\pi}}\left(\frac{6}{N^{2}-1}\right)^{\frac{1}{2}} \frac{N^{l}}{l^{\frac{1}{2}}}\left[1-\frac{3}{2 l}-\frac{13}{1120 l^{2}}-\ldots\right] \quad \text { when } N \gg 1
\end{aligned}
$$

When $k^{\prime}=K l^{\beta}, \beta<\frac{1}{2}$,

$$
\frac{c(N, l, \bar{M})-c(N, l, M)}{c(N, l, \bar{M})} \simeq \frac{3}{2} \frac{K^{2}}{l^{1-2 \beta}}
$$

which is a small fraction when $l$ is large. When $N=g$,

$$
c(N, l, \bar{M})-c\left(N, l, M_{k}\right)=\frac{3}{2} \frac{K^{2}}{\sqrt{\pi}}\left(\frac{6}{g^{2}-1}\right)^{\frac{1}{2}} \frac{g^{l}}{l^{\frac{3}{2}-2 \beta}}
$$

The contribution of the elements $\left\{V_{\alpha} \mid V_{\alpha}=T_{n_{1}}^{ \pm 1} \ldots T_{n_{l}}^{ \pm 1}\right\}$ in the region $\theta_{M} \simeq \theta_{\bar{M}_{l}}$ to $\left|K_{\alpha}\right|^{\frac{1}{2}}$ increases with respect to the genus at least as fast as $\frac{2^{l}}{l^{\frac{3}{2}-2 \beta}} g^{\frac{l}{2}-1}$ implying that a linear bound for the sum cannot be used.

The primitive-element products $\prod_{\alpha}{ }^{\prime}\left|1+K_{\alpha}^{\frac{1}{2}}\right|$ can nevertheless be bounded by refining the estimates of the complex product $\prod_{\alpha}^{\prime}\left(1+K_{\alpha}^{\frac{1}{2}}\right)$.

When $r=r^{\prime}=r^{\prime \prime}$, the distances decrease with $g$ at the same rate, $\chi_{1}, \chi_{2}$ can take a wider range of values, and the arguments $\arg K_{S T}$ will be randomly distributed throughout the interval $[0,2 \pi]$. The fraction of circles for a given value of $r$ can be computed. If the distances between isometric circles belonging to neighbouring levels in a hexagonal configuration range from $\frac{\eta_{0}}{\sqrt{g}}$ and $\frac{\eta_{0}^{\prime}}{\sqrt{g}}$, and the distances between the points $\xi_{1 S}, \xi_{1 T},-\frac{\delta_{S}}{\gamma_{S}},-\frac{\delta_{T}}{\gamma_{T}}$ are greater than $\frac{\epsilon_{0}}{g^{1-2 q^{\prime}}}$ and less than $\frac{\epsilon_{0}^{\prime}}{g^{1-2 q^{\prime}}}$, then the level number differences, corresponding to configurations of two pairs of isometric circles satisfying these constraints on the distances, will obey the inequalities

$$
\frac{\epsilon_{0}}{\eta_{0}^{\prime}} g^{\frac{1}{2}-q^{\prime}}<\Delta l<\frac{\epsilon_{0}^{\prime}}{\eta_{0}} g^{\frac{1}{2}-q^{\prime}}
$$

With $6(\Delta l-1)$ circles in each level and the range of levels given in equation $(2.30)$, the fraction of circles for which the argument can be arbitrary is

$$
\begin{aligned}
& \frac{3\left(\frac{\epsilon_{0}^{\prime 2}}{\eta_{0}^{2}}-\frac{\epsilon_{0}^{2}}{\eta_{0}^{\prime 2}}\right) g^{1-2 q^{\prime}}-3\left(\frac{\epsilon_{0}^{\prime}}{\eta_{0}}-\frac{\epsilon_{0}}{\eta_{0}^{\prime}}\right) g^{\frac{1}{2}-q^{\prime}}}{2 g} \\
& =\frac{3}{2}\left(\frac{\epsilon_{0}^{\prime}}{\eta_{0}^{2}}-\frac{\epsilon_{0}^{\prime}}{\eta_{0}^{\prime 2}}\right) g^{-2 q^{\prime}}-\frac{3}{2}\left(\frac{\epsilon_{0}^{\prime}}{\eta_{0}}-\frac{\epsilon_{0}}{\eta_{0}^{\prime}}\right) g^{-q^{\prime}-\frac{1}{2}}
\end{aligned}
$$


for each value of $q^{\prime}$. Summing over all values of $q_{N}^{\prime}$,

$$
\begin{aligned}
& \sum_{N=0}^{N_{\max }} g^{-q^{\prime}}=\frac{\left[\left(\frac{\epsilon_{0}^{\prime}}{\epsilon_{0}}\right)^{\frac{1}{2}}-\frac{1}{\sqrt{g}}\right]}{\left[\left(\frac{\epsilon_{0}^{\prime}}{\epsilon_{0}}\right)^{\frac{1}{2}}-1\right]} \quad N_{\max }=\left[\frac{\ln g}{\ln \left(\frac{\epsilon_{0}^{\prime}}{\epsilon_{0}}\right)}\right] \\
& \sum_{N=0}^{N_{\max }} g^{-2 q^{\prime}}=\frac{\frac{\epsilon_{0}^{\prime}}{\epsilon_{0}}-\frac{1}{g}}{\frac{\epsilon_{0}^{\prime}}{\epsilon_{0}}-1}
\end{aligned}
$$

so that the ratio of generators $T_{n_{\ell}}$, such that the multiplier with product of two generators has an arbitrary argument, is

$$
\frac{3}{2}\left(\frac{\epsilon_{0}^{\prime}}{\eta_{0}^{2}}-\frac{\epsilon_{0}}{\eta_{0}^{\prime 2}}\right)\left[\frac{\frac{\epsilon_{0}^{\prime}}{\epsilon_{0}}-\frac{1}{g}}{\frac{\epsilon_{0}^{\prime}}{\epsilon_{0}}-1}\right]-\frac{3}{2}\left(\frac{\epsilon_{0}^{\prime}}{\eta_{0}}-\frac{\epsilon_{0}}{\eta_{0}^{\prime}}\right) g^{-\frac{1}{2}} \frac{\left[\left(\frac{\epsilon_{0}^{\prime}}{\epsilon_{0}}\right)^{\frac{1}{2}}-\frac{1}{\sqrt{g}}\right]}{\left[\left(\frac{\epsilon_{0}^{\prime}}{\epsilon_{0}}\right)^{\frac{1}{2}}-1\right]}
$$

For this fraction of circles, the arguments of the multipliers $K_{\alpha}$ will be distributed evenly througout the interval $[0,2 \pi]$, and the terms $\left|1+K_{\alpha}^{\frac{1}{2}}\right|$ can be paired

$$
\left.|1+| K_{\alpha}\right|^{\frac{1}{2}} e^{\frac{i \theta_{\alpha}}{2}}|| 1+\left|K_{\alpha}\right|^{\frac{1}{2}} e^{\frac{i\left(\theta_{\alpha}+\pi\right)}{2}}|=| 1-K_{\alpha} \mid
$$

thus obtaining a bound

$$
\prod_{\left(\alpha, \alpha_{c}\right)}^{\prime}\left|1-K_{\alpha}\right|<\exp \left(\sum_{\alpha, \alpha_{c}}^{\prime}\left|K_{\alpha}\right|\right)
$$

where $K_{\alpha}$ and $K_{\alpha_{c}}$ represent complementary multipliers with phases of opposite sign and $I_{V_{\alpha}}, I_{V_{\alpha_{c}}}$ are isometric circles belonging to the restricted category labelled by the power $r$.

It will be assumed henceforth that the set of isometric circles associated with a product of generators does not belong to this category, so that the argument of the multiplier of a product of generators approximately equals the sum of the arguments of the multipliers of each generator. Between the values 1 and $\left[\frac{4 \pi}{v}\right]$, the factor in the primitive-element product corresponding to the mean value $\bar{M}$ is

$$
|1+| K_{\alpha_{(l)}}\left|\frac{1}{2} e^{i \frac{\theta_{\bar{M}_{l}}}{2}}\right|=\left[1+\left|K_{\alpha_{(l)}}\right|+2\left|K_{\alpha(l)}\right|^{\frac{1}{2}} \cos \frac{l v}{2}\right]^{\frac{1}{2}}
$$

When $l \leq \frac{2 \pi}{v}$, or $\frac{6 \pi}{v} \leq l \leq \frac{8 \pi}{v}, \cos \frac{l v}{2}>0$

$$
\left|1+K_{\alpha_{(l)}}^{\frac{1}{2}}\right| \geq\left[1+\left|K_{\alpha(l)}\right|\right]^{\frac{1}{2}}
$$


whereas when $\frac{2 \pi}{v} \leq l \leq \frac{6 \pi}{v}$

$$
\left|1+K_{\alpha_{(l)}}^{\frac{1}{2}}\right| \leq\left[1+\left|K_{\alpha_{(l)}}\right|\right]^{\frac{1}{2}}
$$

so that an upper bound for the product over elements in the second and third quadrants is obtained.

The angular distribution at each level $l$ can be plotted. The angles are restricted to the range $[0,2 \pi]$, by identifying $\theta$ with $\theta+2 \pi$. For any distribution with mean angle greater than $\pi$, the upper limit of the distribution exceeds $2 \pi$, and the periodicity property is relevant.

Since the number of elements with $\frac{\theta_{\bar{M}_{l}}}{2}$ in the fourth quadrant is considerably larger than the number of elements with arguments $\frac{\theta_{\bar{M}_{l}}}{2}$ in the second quadrant, there are not enough terms $\left|1+K_{\alpha(l)}^{\frac{1}{2}}\right|$ with $\pi \leq \frac{\theta_{\bar{M}_{\ell}}}{2} \leq \frac{3 \pi}{2}$ which can be paired off with terms in the product to obtain a bound involving a product over terms of the form $\left|1-K_{\alpha_{(l)}}\right|$.

Suppose, for example, that the mean of the distribution occurs at $\theta_{\bar{M}_{\ell}}=n \pi$, while the upper limit is $2 n \pi$. Then, the graph in the interval $[(2 n-2) \pi, 2 n \pi]$ is the reflection of the graph in the interval $[0,2 \pi]$. When the distribution functions in the two intervals are superimposed, there will be dip in the intermediate range if $c\left(N, \frac{1}{2}\left[\frac{4 \pi}{v}(N+1)\right]\right)<\frac{1}{2} c\left(N, l, \frac{1}{2}\left[\frac{8 \pi}{v}(N+1)\right]\right)$.

When $M$ is less than the minimum value allowed in the estimate (2.26), the general formula (2.25) is preferable when considering larger deviations from the mean. Specifically, if $M=\lambda \bar{M}$ and $k=\frac{\eta l}{2}, 0 \leq \eta \leq \lambda \leq 1$, then

$$
\begin{aligned}
c(N, l, M) & =\sum_{\{\eta\}}(-1)^{\frac{\eta l}{2}}\left(\begin{array}{c}
l \\
\frac{\eta l}{2}
\end{array}\right)\left(\begin{array}{c}
l-1+\frac{\lambda-\eta}{2} l N+\frac{\lambda l}{2} \\
l-1
\end{array}\right) \\
& \simeq \sum_{\{\eta\}}\left(\frac{-1}{\eta l}\right)^{\frac{\eta l}{2}} \frac{2^{l}}{(2-\eta)^{\left(l-\frac{\eta l}{2}\right)}}(\lambda-\eta)^{l-1} N^{l-1}
\end{aligned}
$$

The term with the maximum value in this sum is determined by the value of $\eta$ satisfying the transcendental equation

$$
1-\eta=\tanh \left(\frac{l-1}{l(\lambda-\eta)}\right)
$$

and the leading dependence on $\lambda$ is $\lambda^{l-1}$. The inequality $c\left(N, l, \frac{1}{2}\left[\frac{4 \pi}{v}(N+1)\right]\right)$ $<\frac{1}{2} c\left(N, l, \frac{1}{2}\left[\frac{8 \pi}{v}(N+1)\right]\right)$ will be satisfied for $l \geq 2$ since $\lambda \doteq 2$. More generally, the distribution function in the interval $[(2 n-2 m-2) \pi,(2 n-2 m) \pi]$ is a reflection of the graph in the interval $[2 m \pi,(2 m+2) \pi]$, and their superposition will produce a dip in the intermediate region only if $c\left(N, l, \frac{1}{2}\left[\frac{(8 m+4) \pi}{v}(N+1)\right]\right)-c\left(N, l, \frac{1}{2}\left[\frac{(8 m) \pi}{v}(N+1)\right]\right)$ $<\frac{1}{2}\left[c\left(N, l, \frac{1}{2}\left[\frac{(8 m+8) \pi}{v}(N+1)\right]\right)-c\left(N, l, \frac{1}{2}\left[\frac{(8 m) \pi}{v}(N+1)\right]\right)\right]$. Continuing the process of superimposing the distribution function in the other intervals onto the interval $[0,2 \pi]$, it follows 
that the final graph is symmetric when the peak of the distribution is located at $n \pi$ and essentially symmetric when the peak is located at another value in the interval $((n-1) \pi,(n+1) \pi)$ with any deviation from symmetry confined to an angular interval with a maximal size approximately equal to $\frac{1}{n}$ times the extent of the original distribution. Initially, there will be more elements $V_{\alpha}$ with multipliers such that $\arg K_{\alpha}^{\frac{1}{2}}$ belong to the first and fourth quadrants rather than the second and third quadrants. The deficit is typically given by $\left(\frac{2 m+2}{l}\right)^{l-1}+\left(\frac{2 m}{l}\right)^{l-1}-2\left(\frac{2 m+1}{l}\right)^{l-1}$ and summing the deficit over the index range [1, [ $\left.\left.\frac{n}{2}\right]\right]$ gives

$$
\begin{aligned}
& \sum_{m=1}^{\left[\frac{n^{\prime}}{2}\right]}\left[\left(\frac{2 m+2}{l}\right)^{l-1}+\left(\frac{2 m}{l}\right)^{l-1}-2\left(\frac{2 m+1}{l}\right)^{l-1}\right] \simeq \frac{2^{l-1}}{l}\left(\frac{l(l-1)}{4}\left[\frac{n^{\prime}}{2}\right]^{l-2}+\ldots\right) \\
& =2^{l-3}(l-1)\left[\frac{n^{\prime}}{2}\right]^{l-2}
\end{aligned}
$$

with $n^{\prime}=\left[\frac{l-K l^{\frac{1}{2}}}{\left[\frac{4 \pi}{v}\right]}\right]$

Two separate sums based on the formula (2.22) for the composition function $c(N, l, M)$ must be added to the overall deficit (2.41). If the interval $\left[\left[\frac{\left(l-2 k^{\prime \prime}-1\right)}{\left[\frac{4 \pi}{v}\right]}\right] \pi,\left[\frac{\left(l-2 k^{\prime \prime}+1\right)}{\left[\frac{4 \pi}{v}\right]}\right] \pi\right]$ is mapped onto $[0,2 \pi]$, then after summing over $k^{\prime \prime}$, the surplus is

$$
\begin{gathered}
-\sum_{k^{\prime \prime}=\left\{\frac{K l \beta}{2}\right\}}^{2} \frac{1}{\sqrt{\pi}}\left(\frac{6}{N^{2}-1}\right)^{\frac{1}{2}} \frac{N^{l}}{l^{\frac{1}{2}}} \frac{3}{2 l} \frac{N+1}{N-1}\left[2 k^{\prime 2}-\left(k^{\prime}+1\right)^{2}-\left(k^{\prime}-1\right)^{2}\right] \\
=\frac{2}{\sqrt{\pi}}\left(\frac{6}{N^{2}-1}\right)^{\frac{1}{2}} \frac{N+1}{N-1} \frac{3 N^{l}}{2 l^{\frac{3}{2}}}\left(K l^{\beta}-2\right)
\end{gathered}
$$

and therefore it is suppressed relative to the magnitude of $c(N, l, M)$ in this interval by a factor of $l^{1-\beta}$, and the same result is obtained if $\left[\left[\frac{\left(l-2 k^{\prime \prime}\right)}{\left[\frac{4 \pi}{v}\right]}\right] \pi,\left[\frac{\left(l-2 k^{\prime \prime}+2\right)}{\left[\frac{4 \pi}{v}\right]}\right] \pi\right]$ is superimposed on $[0,2 \pi]$.

When the peak of the distribution is located at the angular value $n \pi$ for $n$ even, the interval $[(n-2) \pi, n \pi]$ is mapped on $[0,2 \pi]$, then the surplus can be computed by extending the sum to $k^{\prime \prime}=1$. If the peak is located at the angular value $n \pi$, for $n$ odd, then the surplus is essentially given by the difference between the peak and the value at $(n-1) \pi$, which would equal $\frac{1}{\sqrt{\pi}}\left(\frac{6}{N^{2}-1}\right)^{\frac{1}{2}} \frac{N+1}{N-1} \frac{3 N^{l}}{2 l^{\frac{3}{2}}}$. This magnitude is suppressed by a factor of $\frac{1}{l}$ with respect the average value of $c(N, l, M)$ in the interval. As $l$ increases, the weight given to each element of the Schottky group at level $l$ decreases more quickly than $\left(\frac{\epsilon_{0}^{\prime}}{g^{1-2 q^{\prime}}}\right)^{\frac{l}{2}}$ and combining this weighting with equation (2.42) gives a genus-dependence of $\epsilon_{0}^{\prime l} g^{\frac{l}{2}-1+q^{\prime} l}$. However, as $l$ increases, the 
peak of the distribution also moves from the midpoint of the interval $[(n-1) \pi,(n+1) \pi]$ and over the index range $l=n\left[\frac{4 \pi}{v}\right], \ldots,(n+1)\left[\frac{4 \pi}{v}\right]$, the argument will be shifted sufficiently to cause a cancellation in the phases of $K_{\alpha}(l)$, implying an approximately uniform distribution function throughout the entire interval $[0,2 \pi]$.

An upper bound for $\prod_{\alpha}{ }^{\prime}\left|1+K_{\alpha}^{\frac{1}{2}}\right|^{-1}$ is equivalent to a lower bound for the product $\prod_{\alpha}{ }^{\prime}\left|1+K_{\alpha}^{\frac{1}{2}}\right|$, and although an infimum has not been obtained for $\prod_{\alpha}{ }^{\prime}\left|1+K_{\alpha}^{\frac{1}{2}}\right|^{-1}$ in the range $\left\{\frac{\pi}{v}\right\} \leq l \leq\left[\frac{3 \pi}{v}\right]$, the elements $V_{\alpha(l)}, \frac{\theta_{\alpha(l)}}{2} \in\left[\frac{\pi}{2}, \frac{3 \pi}{2}\right]$ can be paired with the elements $V_{\alpha}$, such that $\arg K_{\alpha}^{\frac{1}{2}}$ belongs to the first or fourth quadrant, giving products of the form

$$
\prod_{\left(\alpha, \alpha_{c}\right)}^{\prime}\left|1-K_{\alpha}\right| \geq \exp \left(-\sum_{\left(\alpha, \alpha_{c}\right)}{ }^{\prime}\left|K_{\alpha}\right|\right)
$$

so that an exponential lower bound for the product $\prod_{\alpha}{ }^{\prime}\left|1+K_{\alpha}^{\frac{1}{2}}\right|$ exists.

An exponential bound is immediately obtained in the limit $\left|K_{n}\right| \rightarrow 0$ since all of the primitive-element products tend to 1 . The fixed-point distance limit $\left|\xi_{1 n}-\xi_{2 n}\right| \rightarrow 0$ could only have an effect on $[\operatorname{det}(\operatorname{Im} \mathcal{T})]^{-5}$, and as $Z_{1 n} \rightarrow Z_{2 n}, \frac{Z_{1 m}-V_{\alpha} Z_{1 n}}{Z_{1 m}-V_{\alpha} Z_{2 n}} \frac{Z_{2 m}-V_{\alpha} Z_{2 n}}{Z_{2 m}-V_{\alpha} Z_{1 n}} \rightarrow 1$, so that each of the sums and products over conjugacy classes receive a contribution which can be bounded by an exponential function of the genus.

\section{Estimates of Moduli Space Integrals corresponding to Closed Surfaces}

Combining the holomorphic part of the measure with its complex conjugate gives a multiplier integral with a dominant part

$$
\int \frac{d\left|K_{n}\right|}{\left|K_{n}\right|\left(\ln \left(\frac{1}{\left|K_{n}\right|}\right)\right)^{5}}=\frac{1}{4}\left[\ln \left(\frac{1}{\left|K_{n}\right|}\right)\right]^{-4}
$$

in the limit $\left|K_{n}\right| \rightarrow 0$. With the limits in categories (i) and (ii), the integral gives a factor

$$
\left(\left(1-2 q^{\prime}\right) \ln g\right)^{-5} \ln \left(\frac{\epsilon_{0}^{\prime}}{\epsilon_{0}}\right)+O\left(\left(\left(1-2 q^{\prime}\right) \ln g\right)^{-6}\right)
$$

For the third category of isometric circles, a factor of

$$
\frac{1}{4}\left[\left(\ln \frac{1}{\epsilon_{0}^{\prime}}\right)^{-4}-\left(\ln \frac{1}{\epsilon_{0}}\right)^{-4}\right]
$$

is obtained. 
Instead of integrating over the super-fixed points, it is useful to use another set of coordinates on supermoduli space $\left\{K_{n}, B_{m}, H_{m}, \theta_{1 i}, \theta_{2 i}\right\}$ for which the holomorphic part of the integral over the super-fixed points ${ }^{9}$ is replaced by

$$
\prod_{m=2}^{g} \frac{d B_{m}}{B_{m}^{\frac{3}{2}}} \prod_{m=2}^{g-1} \frac{d H_{m}}{H_{m}^{\frac{3}{2}}} \prod_{i=2}^{g-1} d \vartheta_{1 i} \prod_{i=1}^{g} d \vartheta_{2 i}
$$

where

$$
\begin{aligned}
& \xi_{2 n}=\prod_{j=2}^{n} B_{j} \quad \theta_{2 n}=\frac{1}{\sqrt{\xi_{2 n}}} \vartheta_{2 n} \quad \theta_{2 g}=\frac{1}{\sqrt{\xi_{2 g}}} \vartheta_{2 g} \\
& \xi_{1 n}=\frac{\xi_{2 n}}{1-H_{n}-\sqrt{H_{n}} \vartheta_{1 n} \vartheta_{2 n}} \quad \theta_{1 n}=\frac{\sqrt{H_{n} \xi_{2 n}} \vartheta_{1 n}+\theta_{2 n}}{1-H_{n}}
\end{aligned}
$$

The relation involving $H_{n}$ can be inverted to give

$$
\begin{aligned}
H_{n}=\frac{\xi_{1 n}-\xi_{2 n}}{\xi_{1 n}}- & \frac{\left(\xi_{1 n}-\xi_{2 n}\right)^{\frac{1}{2}}}{\xi_{1 n}^{\frac{1}{2}}} \vartheta_{1 n} \vartheta_{2 n} \\
\left|H_{n}\right|^{-1}=\left|\frac{\xi_{1 n}}{\xi_{1 n}-\xi_{2 n}}\right|[1 & +\frac{1}{2} \frac{\xi_{1 n}^{\frac{1}{2}}}{\left(\xi_{1 n}-\xi_{2 n}\right)^{\frac{1}{2}}} \vartheta_{1 n} \vartheta_{2 n} \\
& +\frac{1}{2} \frac{\bar{\xi}_{1 n}^{\frac{1}{2}}}{\left(\xi_{1 n}-\xi_{2 n}\right)^{\frac{1}{2}}} \bar{\vartheta}_{1 n} \bar{\vartheta}_{2 n} \\
& \left.+\frac{1}{4} \frac{\left|\xi_{1 n}\right|}{\left|\xi_{1 n}-\xi_{2 n}\right|} \vartheta_{1 n} \vartheta_{2 n} \bar{\vartheta}_{1 n} \bar{\vartheta}_{2 n}\right]
\end{aligned}
$$

The last component of this expression absorbs all except four of the integrals over the Grassmann variables. Examples of graviton scattering show that the $\vartheta_{21}, \vartheta_{2 g}, \bar{\vartheta}_{21}, \bar{\vartheta}_{2 g}$ integrals are non-zero because the Green function on the super-Riemann surface depends on all of the elements of the super-Schottky group. ${ }^{10,13}$ The first term in the integral over $\left|H_{n}\right|$ produces a dependence on the fixed-point distance of $\left|\xi_{1 n}-\xi_{2 n}\right|^{-1}$, whereas the dominant contribution to the integral with Grassmann variables is given by $\left|\xi_{1 n}-\xi_{2 n}\right|^{-2}$. The contribution of a configuration of isometric circles with $N_{0}$ circles belonging to either of the first two categories and $g-N_{0}$ circles in the third category to the multiplier integral is

$$
\begin{aligned}
& \prod_{m=1}^{N_{0}} \ln \left(\frac{\epsilon_{0}^{\prime}}{\epsilon_{0}}\right)^{N_{0}}(\ln g)^{-5 N_{0}} \\
& \cdot\left[\frac{1}{4} \ln \left(\frac{\epsilon_{0}^{\prime}}{\epsilon_{0}}\right)\left(\ln \frac{1}{\epsilon_{0}}\right)^{-4}\left(\ln \frac{1}{\epsilon_{0}^{\prime}}\right)^{-4}\right. \\
& \left.\quad\left(\ln \frac{1}{\epsilon_{0}}+\ln \frac{1}{\epsilon_{0}^{\prime}}\right)\left(\left(\ln \frac{1}{\epsilon_{0}}\right)^{2}+\left(\ln \frac{1}{\epsilon_{0}^{\prime}}\right)^{2}\right)\right]^{g-N_{0}}
\end{aligned}
$$


In this formula, $\bar{q}^{\prime}$ represents a weighted average of the values $q_{m}^{\prime}$ associated with the $N_{0}$ isometric circles in the first two categories. There are

$$
\frac{\left(N_{0}+\frac{(\ln g)^{2}}{2\left(\ln \left(\frac{\epsilon_{0}^{\prime}}{\epsilon_{0}}\right)\right)^{2}}-1\right) !}{N_{0} !\left(\frac{(\ln g)^{2}}{2\left(\ln \left(\frac{\epsilon_{0}^{\prime}}{\epsilon_{0}}\right)\right)^{2}}-1\right) !}
$$

different partitions of the $N_{0}$ circles into the $\frac{(\ln g)^{2}}{2\left(\ln \left(\frac{\epsilon_{0}^{\prime}}{\epsilon_{0}}\right)\right)^{2}}$ subcategories labelled by the indices $q$ and $q^{\prime}$, and each partition $\left\{n_{i}\right\}$ is weighted by a factor $\frac{1}{n_{1} ! \ldots n_{r} !}$, with $n_{1}+\ldots+n_{r}=N_{0}$, since one set of inequalities defining the fundamental domain of the modular group, $(\operatorname{Im} \tau)_{s s} \geq$ $(\operatorname{Im} \tau)_{r r}, s \geq r$, must be satisfied for each subcategory, leading to restrictions on the ranges of the multipliers. ${ }^{2}$

For the parameters associated with the $g-N_{0}$ remaining isometric circles in the third category, the constraints $(\operatorname{Im} \tau)_{s s} \geq(\operatorname{Im} \tau)_{r r}, s \geq r$, lead to a reduction of the integral by a factor of $\left(g-N_{0}\right)$ !. After summing over the possible values of $N_{0}$, the combination of the integrals over the multipliers $K_{n}$ and $H_{n}$, with the weighting factors, is bounded above by

$$
\begin{gathered}
\sum_{N_{0}=0}^{g} \sum_{\substack{\left\{n_{i}\right\} \\
\sum_{i} n_{i}=N_{0}}} \frac{1}{n_{1} ! \ldots n_{r} !}\left(1-2 \bar{q}^{\prime}\right)^{N_{0}}\left[\ln \frac{\epsilon_{0}^{\prime}}{\epsilon_{0}}\right]^{N_{0}}(\ln g)^{-5 N_{0}} g^{2 \sum_{i} q_{i} n_{i}} \\
\cdot\left[\frac{1}{4} \ln \left(\frac{\epsilon_{0}^{\prime}}{\epsilon_{0}}\right)\left(\ln \frac{1}{\epsilon_{0}}\right)^{-4}\left(\ln \frac{1}{\epsilon_{0}^{\prime}}\right)^{-4}\left(\ln \frac{1}{\epsilon_{0}}+\ln \frac{1}{\epsilon_{0}^{\prime}}\right)\left(\left(\ln \frac{1}{\epsilon_{0}}\right)^{2}+\left(\ln \frac{1}{\epsilon_{0}^{\prime}}\right)^{2}\right)\right]^{g-N_{0}} \\
\left(1-2 \bar{q}^{\prime}\right)^{N_{0}}=\prod_{m=1}^{N_{0}}\left(1-2 q_{m}^{\prime}\right)
\end{gathered}
$$

The combinatorial factor $\frac{g^{2} \sum_{i}{ }^{{ }{ }_{i}{ }_{i}} g^{g-N_{0}}}{n_{1} ! \ldots n_{r} !\left(g-N_{0}\right) !}$ obtaines a maximum value when $r=1$ and $N_{0}=\frac{g}{2}$. If all of the $N_{0}$ isometric circles belong to the subcategory defined by the value $q_{i}=\frac{1}{2}-\frac{\ln \frac{\epsilon_{0}^{\prime}}{\epsilon_{0}}}{\ln g}$ then the factor is approximately equal to $\left(\frac{2 \cdot e \cdot \epsilon_{0}}{\epsilon_{0}^{\prime}}\right)^{g}$.

Re-expressing the part of the measure containing the multipliers $\left\{B_{m}\right\}$ as

$$
\prod_{m=2}^{g} \frac{d B_{m}}{B_{m}^{\frac{3}{2}}} \cdot \prod_{m=2}^{g} \frac{d \bar{B}_{m}}{\bar{B}_{m}^{\frac{3}{2}}}=\prod_{m=2}^{g} \frac{\left|B_{m}\right| d\left|B_{m}\right| d \theta_{m}^{B}}{\left|B_{m}\right|^{3}}
$$


where $\theta_{m}^{B}$ is the angular coordinate of this variable, it follows if the range for $\left|B_{j}\right|$ is $\left[\tilde{\epsilon}, \delta_{2}^{\prime \frac{1}{g}}\right]$ or equivalently $\left[\tilde{\epsilon}, 1+\frac{\ln \delta_{2}^{\prime}}{g}\right]$ if $\delta_{2}^{\prime}>1$, the integral is bounded by

$$
(2 \pi)^{g-1} \prod_{m=2}^{g} \int_{\tilde{\epsilon}}^{1+\frac{\ln \delta_{2}^{\prime}}{g}} \frac{d\left|B_{m}\right|}{\left|B_{m}\right|^{2}}=(2 \pi)^{g-1} \prod_{m=2}^{g}\left[\frac{1}{\tilde{\epsilon}}-\frac{1}{1+\frac{\ln \delta_{2}^{\prime}}{g}}\right]<\left(\frac{2 \pi}{\tilde{\epsilon}}\right)^{g-1}
$$

The division by $\left(g-N_{0}\right)$ ! rather than $\frac{\left(g-N_{0}\right)}{J}$ ! leads to an upper bound depending exponentially on the genus for all $N_{0}$, as the combinatorial factor is less than

$$
\begin{aligned}
& g^{N_{0}} \quad \text { when } \quad N_{0} \leq \frac{(\ln g)^{2}}{2\left(\ln \frac{\epsilon_{0}^{\prime}}{\epsilon_{0}}\right)^{2}} \\
& e^{\left(1-2 \frac{\ln \left(\frac{(\ln g)}{\ln \frac{\epsilon_{0}^{\prime}}{\epsilon_{0}}}\right)}{\ln g}\right)} \text { when } \frac{(\ln g)^{2}}{2\left(\ln \left(\frac{\epsilon_{0}^{\prime}}{\epsilon_{0}}\right)^{2}\right)} \leq \frac{g}{\ln g} \\
& {\left[\left(1+\frac{\ln \lambda}{\lambda}+\frac{1}{\lambda}\right) \cdot e\right]^{g} \cdot\left(\frac{(\ln g)^{2}}{2\left(\ln \frac{\epsilon_{0}^{\prime}}{\epsilon_{0}}\right)^{2}}\right) ! \quad \text { when } \quad \frac{g}{\ln g} \leq N_{0} \leq \frac{g}{\lambda}, \lambda \gg 1} \\
& \left(\frac{2 \cdot e \cdot \epsilon_{0}}{\epsilon_{0}^{\prime}}\right)^{g} \quad \text { when } \quad \frac{g}{\lambda} \leq N_{0} \leq \frac{g}{2}
\end{aligned}
$$

Each of these upper bounds must be multiplied by the number of partitions (3.8) for each 
value of $N_{0}$, and since this is less than $(\ln g)^{2 N_{0}}$, the sum (3.7) is bounded above by

$$
\begin{aligned}
& \frac{1}{4^{g}}\left[\left(\ln \frac{1}{\epsilon_{0}^{\prime}}\right)^{-4}-\left(\ln \frac{1}{\epsilon_{0}}\right)^{-4}\right]^{g}\left[\left(1+\frac{\ln \lambda}{\lambda}+\frac{1}{\lambda}\right) \cdot e\right]^{g} \cdot\left(\frac{(\ln g)^{2}}{2\left(\ln \frac{\epsilon_{0}^{\prime}}{\epsilon_{0}}\right)^{2}}\right) ! \\
& {\left[1-4\left(1-2 \bar{q}^{\prime}\right)\left(\ln \frac{1}{\epsilon_{0}}\right)^{4}\left(\ln \frac{1}{\epsilon_{0}^{\prime}}\right)^{4}\right.} \\
& \left.\cdot\left(\ln \frac{1}{\epsilon_{0}}+\ln \frac{1}{\epsilon_{0}^{\prime}}\right)^{-1}\left(\left(\ln \frac{1}{\epsilon_{0}}\right)^{2}+\left(\ln \frac{1}{\epsilon_{0}^{\prime}}\right)^{2}\right)^{-1}(\ln g)^{-3}\right]^{-1} \\
& +\frac{1}{4^{g}}\left[\left(\ln \frac{1}{\epsilon_{0}^{\prime}}\right)^{-4}-\left(\ln \frac{1}{\epsilon_{0}}\right)^{-4}\right]^{g}\left(\frac{2 \cdot e \cdot \epsilon_{0}}{\epsilon_{0}^{\prime}}\right)^{g} g\left(1-\frac{1}{\lambda}\right) \\
& {\left[4\left(1-2 \bar{q}^{\prime}\right)\left(\ln \frac{1}{\epsilon_{0}}\right)^{4}\left(\ln \frac{1}{\epsilon_{0}^{\prime}}\right)^{4}\left(\ln \frac{1}{\epsilon_{0}}+\ln \frac{1}{\epsilon_{0}^{\prime}}\right)^{-1}\left(\left(\ln \frac{1}{\epsilon_{0}}\right)^{2}+\left(\ln \frac{1}{\epsilon_{0}^{\prime}}\right)^{2}\right)^{-1}\right.} \\
& \left.[\ln g)^{3}\right]^{-\frac{g}{\lambda}}
\end{aligned}
$$

As $1 \gg \frac{1}{\lambda}>0$, the second term in the bound (3.13) is a rapidly decreasing function of the genus for large $g$, while the exponential dependence of the first term on the genus is determined by $\epsilon_{0}^{\prime}$. This parameter is constrained by modular invariance. One of the conditions defining the fundamental region of the modular group is $|\operatorname{det}(C \tau+D)| \geq 1$ for $\left(\begin{array}{ll}A & B \\ C & D\end{array}\right) \in S p(2 g ; \mathbb{Z})$. Since

$$
|\operatorname{det}(C \tau+D)|=|\operatorname{det}(\operatorname{Im} \tau)| \mid \operatorname{det}\left(C-i C(\operatorname{Re} \tau)(\operatorname{Im} \tau)^{-1}-i D(\operatorname{Im} \tau)^{-1} \mid\right.
$$

the determinant will be greater than one for all C, D when $|\operatorname{det}(\operatorname{Im} \tau)| \geq b>1$ for some number $b$, so that $\left|\operatorname{det}\left(C-i C(\operatorname{Re} \tau)(\operatorname{Im} \tau)^{-1}-i D(\operatorname{Im} \tau)^{-1}\right)\right|$ will be bounded below when $\operatorname{det} C \neq 0$ and equal to $|\operatorname{det} D|\left|\operatorname{det}(\operatorname{Im} \tau)^{-1}\right| \geq|\operatorname{det}(\operatorname{Im} \tau)|^{-1}$ when $C=0$. Moreover, the minimum value of $\frac{\operatorname{tr}(\operatorname{Im} \tau)}{g}$ is

$$
\ln \frac{1}{\epsilon_{0}^{\prime}}-\frac{1}{g} \sum_{n=1}^{g} s_{n n}
$$

$$
s_{n n} \text { is a least upper bound for } \sum_{\alpha}{ }^{(n, n)} \ln \left|\frac{\xi_{1 n}-V_{\alpha} \xi_{2 n}}{\xi_{1 n}-V_{\alpha} \xi_{1 n}} \frac{\xi_{2 n}-V_{\alpha} \xi_{1 n}}{\xi_{2 n}-V_{\alpha} \xi_{2 n}}\right|
$$

implying that the restriction to a fundamental domain requires

$$
\ln \frac{1}{\epsilon_{0}^{\prime}} \geq b^{\frac{1}{g}}+\frac{1}{g} \sum_{n=1}^{g} s_{n n}
$$

Thus, $\left[\left(\ln \frac{1}{\epsilon_{0}^{\prime}}\right)^{-4}-\left(\ln \frac{1}{\epsilon_{0}}\right)^{-4}\right]<1$ and the first term in the bound (3.13) decreases exponentially. From this result, it follows that the sum over genus of the moduli space integrals 
defined by the class of Riemann surfaces associated with the first three categories of isometric circles is finite. Finiteness of these integrals at large orders in perturbation theory contrasts with the rapid divergence of the regularized vacuum amplitude in closed bosonic string theory. 2

Suppose that the bounds for the multiplier integrals in the degeneration limits are $B_{K}, B_{H}$ and $B_{B}$ respectively, whereas the integrals of non-degenerate moduli over the interior of moduli space would have a different set of bounds $B_{K}^{\prime}, B_{H}^{\prime}$ and $B_{B}^{\prime}$. Including all degeneration limits of the A-cycles, B-cycles and C-cycles, an upper bound of

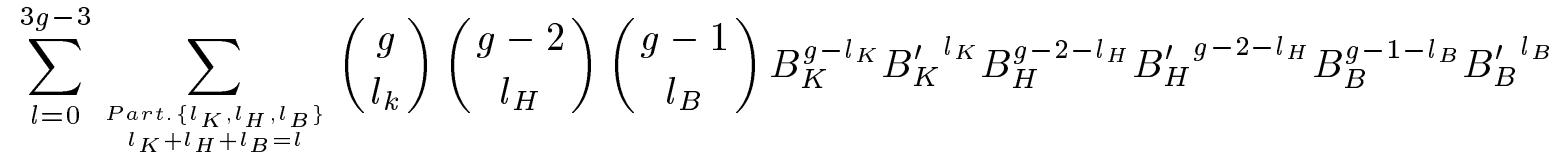

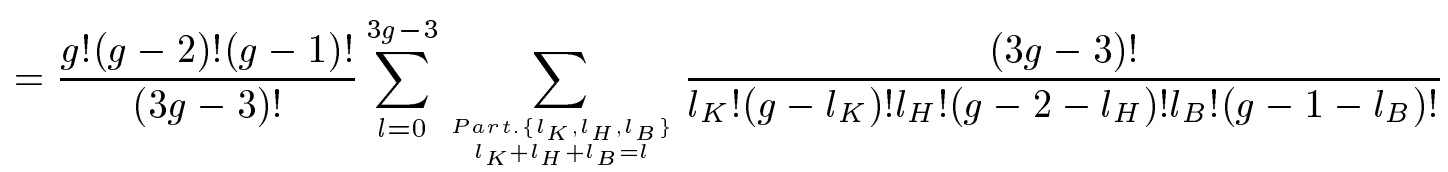

$$
\begin{aligned}
& \times B_{K}^{g-l_{K}} B_{K}^{\prime{ }^{l_{K}}} B_{H}^{g-2-l_{H}} B_{H}^{\prime l_{H}} B_{B}^{g-1-l_{B}} B_{B}^{l_{B}} \\
& <\frac{g !(g-2) !(g-1) !}{(3 g-3) !}\left(B_{K}+B_{K}^{\prime}+B_{H}+B_{H}^{\prime}+B_{B}+B_{B}^{\prime}\right)^{3 g-3}
\end{aligned}
$$

is valid for superstring amplitudes. ${ }^{19}$

The bounds obtained above depended essentially on the restriction of the integral over super-Schottky group parameter space to a single fundamental domain of the super-modular group. In this connection, one may note that the super-mapping class group does not involve any discrete transformations in the odd directions. ${ }^{20}$ The relevant constraints are those listed for the multipliers and ordinary fixed points. Moreover, conditions such as $-\frac{1}{2} \leq(\operatorname{Re} \tau)_{m n} \leq \frac{1}{2}$ and $(\operatorname{Im} \tau)_{1 n} \geq 0$ reduce the integrals by an exponential function of the genus. ${ }^{21}$ Other exponential factors arise from the angular integrations of the arguments of $K_{n}$ and $\xi_{1 n}-\xi_{2 n}$, the integrations over $\xi_{2 n}$ and the primitive-element products. Although the exponents in the primitive-element products in this measure differ from those in the bosonic string measure, the angular integrals again will be bounded by an exponential function of the genus.

Even though the Euler character of moduli space grows asymptotically as $(-1)^{g} \frac{(2 g-1) !}{2^{2 g-1} \pi^{2 g}},{ }^{22}$ the genus-dependence of the volume is affected to a greater extent by the choice of measure. In contrast to the rapid growth of the volume following integration of the bosonic string measure over a subset of moduli space, ${ }^{1}$ the genus-dependence of the corresponding integral is changed significantly by use of the superstring measure (3.4).

The integral over all of moduli space in superstring scattering amplitudes would differ in the large-genus limit from the estimates based on these subdomains by a contribution from the divisor. This, in turn, has been related to tadpoles of massless physical states at lower 
genera ${ }^{23}$. Tadpole diagrams vanish in stable vacua and more generally, if an exponential dependence on the genus of the scattering amplitudes is assumed up to genus $g$ - 1 , the contribution from the boundary of moduli space should also be bounded by a function of the same order, and it follows that this dependence would continue to hold at genus $g$. By induction, the exponential dependence should then be valid for arbitrarily large genus. Finally, there exist other formalisms which avoid the multi-loop ambiguity although they are not directly related to the approach based on a super-Schottky parametrization of supermoduli space. In the light-cone supersheet formalism, the boundary in supermoduli space is determined by the requirement that the bosonic moduli are pure complex numbers without nilpotent parts, eliminating the ambiguity arising from integration over Grassmann variables ${ }^{24}$ Finite, unambiguous scattering amplitudes can also be defined in the twistor-string formalism, which makes use of space-time supersymmetry generators that are independent of the bosonized super-reparametrization ghost fields having poles given by total derivatives in moduli space, 25 and it has been suggested previously that the exponential dependence on the genus could be derived after considering the various degeneration limits of these amplitudes.

\section{Sum over Spin Structures and the Genus-Dependence of Superstring Scattering Amplitudes}

The genus-dependence of the superstring path integral with the measure for an even spin structure will now be obtained, including the contribution of surfaces near the degeneration locus. The use of this measure initially reduces the divergence from $\int \frac{d|K|}{|K|^{3}(\log |K|)^{13}}$ for bosonic strings to $\int \frac{d|K|}{|K|^{2}(\log |K|)^{5}}$ for the Neveu-Schwarz string as $|K| \rightarrow 0$, reflecting the existence of a tachyon in both cases and a shift in the value of the square of its momentum. ${ }^{26}$ It has been noted, however, that an extra factor of $K^{\frac{1}{2}}$ in the holomorphic part of the measure arises for Ramond fermions circulating in the loops, and also when the GSO projection is applied to the Neveu-Schwarz sector. A sum over spin structures, weighted with a phase factor $(-1)^{B_{n}}$, introduces the factors $K_{n}^{\frac{1}{2}}, n=1, \ldots, g$, since

$$
\frac{1}{\left[1-K_{n}^{\frac{1}{2}}\right]^{2}}-\frac{1}{\left[1+K_{n}^{\frac{1}{2}}\right]^{2}}=\frac{4 K_{n}^{\frac{1}{2}}}{\left[1-K_{n}\right]^{2}}
$$

signalling the absence of a tachyon singularity.

At genus 1, where the sum over all four spin structures is required for the vanishing of the one-loop partition function ${ }^{27}$

$$
Z_{1}(\tau)=\frac{1}{2} \frac{1}{\eta(\tau)^{4}}\left[\eta_{(++)} \theta_{1}^{4}(0 \mid \tau)+\eta_{(+-)} \theta_{2}^{4}(0 \mid \tau)+\eta_{(--)} \theta_{3}^{4}(0 \mid \tau)+\eta_{(-+)} \theta_{4}^{4}(0 \mid \tau)\right]
$$

infinities in the limit $\tau \rightarrow i \infty$ cancel amongst the Neveu-Schwarz spin structures $(-+)$ and $(--)$

$$
\lim _{\tau \rightarrow i \infty}\left[\frac{\theta_{3}^{4}(0 \mid \tau)}{\eta(\tau)^{4}}-\frac{\theta_{4}^{4}(0 \mid \tau)}{\eta(\tau)^{4}}\right]=0
$$


The problem of estimating the full superstring amplitude becomes one of summing over all $2^{2 g}$ spin structures, beginning with the set of $2^{g}$ spin structures associated with the NeveuSchwarz sector of the string. Since the sets of odd and even spin structures each form a representation of the modular group, and modular transformations of the Neveu-Schwarz sector can span only the set of even spin structures, it will be necessary to enlarge the original set of $2^{g}$ spin structures to include another sector with odd spin structures.

If $\eta_{(i)}$ denotes the phase associated with the spin structure (i) and $\sigma_{r}$ is the modular transformation, the superstring amplitude $A_{N, g}=\int_{F_{g}} I_{N, g}$, where $F_{g}$ is the fundamental region of the modular group, is invariant so that

$$
\int_{F_{g}} \sum_{(i)=1}^{2^{2 g}} \eta_{(i)} I_{N, g}^{(i)}=\int_{\sigma_{r}\left(F_{g}\right)} \sum_{(i)=1}^{2^{2 g}} \eta_{\sigma_{r}(i)} I_{N, g}^{\sigma_{r}(i)}
$$

This sum can be arranged into sets, each consisting of $2^{g}$ spin structures, so that the sum over spin structures within any individual set introduces a factor of $K_{n}^{\frac{1}{2}}$, in the same manner as the sum over spin structures (4.1) in the Neveu-Schwarz sector, implying removal of the tachyon singularity. Denoting the sets as $S_{r}, r=1,2,3, \ldots$, where $S_{1}$ represents the NeveuSchwarz sector, it can be shown that there exist sets $S_{r}, r=2, \ldots, g$, containing $2^{g-1}$ even and $2^{g-1}$ odd spin structures. In particular, if the signs associated with the A-cycles are chosen to be other than all negative, then an arbitrary choice of signs for the B-cycles produces $2^{g-1}$ even and $2^{g-1}$ odd spin structures. An examination of the signs reveals that these sets of $2^{g-1}$ even and $2^{g-1}$ odd spin structures are required for cancellation of the tachyon divergence, rather than sets consisting only of $2^{g}$ even or $2^{g}$ odd structures.

The sector $S_{2}$ may be chosen to be the Ramond sector, consisting of genus-1 components with either an odd spin structure $(++)$ or an even spin structure $(+-)$. Modular transformations might then be used to generate the remaining $2^{g-1}\left(2^{g}-2\right)$ odd spin structures, and simultaneously, $2^{g-1}\left(2^{g}-2\right)$ spin structures. Labelling of the sectors $\left\{S_{r}\right\}$ is provided in the appendix for genus 1, 2 and 3, although the property of an equal number of even and odd spin structures in $S_{r}, r \geq 2$ holds for arbitrary genus.

The existence of a modular transformation mapping the set $S_{2}$ into $S_{r}, r=3,4, \ldots, g$ should follow from the absence of the tachyon singularity for each of the sets. The tachyon divergences in the limit $\left|K_{n}\right| \rightarrow 0$ cancel only when the correct choice of spin structures, consistent with modular transformations of the integrand corresponding to $S_{2}$, is used. This result should also follow because the symplectic modular group can be generated by the following set of transformations ${ }^{28}$

$$
\begin{aligned}
\text { (i) } A_{n} & \leftrightarrow A_{n^{\prime}} \quad B_{n} \leftrightarrow B_{n^{\prime}} \\
\text { (ii) } B_{n} & \rightarrow B_{n}+A_{n} \\
\text { (iii) } B_{n} & \rightarrow B_{n}+A_{n^{\prime}} \quad B_{n^{\prime}} \rightarrow B_{n^{\prime}}+A_{n} \\
\text { (iv) } B_{n} & \rightarrow A_{n} \quad A_{n} \rightarrow-B_{n}
\end{aligned}
$$


The last transformation switching A-cycles and B-cycles can be used to move the required spin structures from a set which is certainly a modular transformation of $S_{2}$ to one of the sets $S_{r}$ with which it may overlap. It should be possible to transfer all of the spin structures to these sets by repeating this process.

However, instead of explicitly determining the modular transformation mapping $S_{2}$ into $S_{r}$, it is first simpler to consider transformation $\left\{\rho_{n}\right\}$, which are products of genus-one modular transformations acting on the different handles. These modular transformations and the sectors that they generate at genus 2 and 3 have been listed in the appendix. Defining $S_{1}=S_{1}^{\prime}=$ $N S, S_{2}=S_{2}^{\prime}=R$ and $S_{r}^{\prime}=\rho_{r}(R), r=3, \ldots, 3^{g}-2^{g}+1$ the following lemma holds:

Lemma. Consider the Ramond sector $\mathrm{R}$ and the modular transformations $\rho_{r}$ such that $\rho_{r}(R)=S_{r}^{\prime}, r=3, \ldots, 3^{g}-2^{g}+1$, with $\left\{S_{r}^{\prime}\right\}$ not including any Neveu-Schwarz spin structures. The tachyon divergences cancel in each of the sets $S_{r}^{\prime}$.

Proof. The proof follows by induction. The Ramond sector $\mathrm{R}$ at genus 3 is

$$
\begin{array}{cccccc}
+ & + & + & + & + & - \\
+ & + & + & - & + & - \\
+ & - & + & + & + & - \\
+ & - & + & - & + & - \\
+ & + & + & + & + & + \\
+ & + & + & - & + & + \\
+ & - & + & + & + & + \\
+ & - & + & - & + & +
\end{array}
$$

A genus-1 modular transformation could, for example, map the spin structures for the third handle $(++) \rightarrow(++)$ and $(+-) \rightarrow(-+)$ or $(--)$. When this spin structure is not $(++)$, it can be denoted as $i_{h_{3}}$. Application of the modular transformation to the Ramond sector gives the following sector

$$
\begin{array}{ccccc}
+ & + & + & + & i_{h_{3}} \\
+ & + & + & - & i_{h_{3}} \\
+ & - & + & + & i_{h_{3}} \\
+ & - & + & - & i_{h_{3}} \\
+ & + & + & + & (++) \\
+ & + & + & - & (++) \\
+ & - & + & + & (++) \\
+ & - & + & - & (++)
\end{array}
$$

This sector therefore can be divided into two sets corresponding to the spin structures for the third handle being $i_{h_{3}}$ and $(++)$. The genus-2 components are identical and represent the genus-2 Ramond sector. As the spin structures match and the tachyon divergences cancel for the genus-2 Ramond sector, the same property will hold for the genus- 3 sector above.

At genus $g$, a combination of genus-1 modular transformations applied to the Ramond 
sector $R(g)$ produces

$$
\begin{array}{ccccccccccccccccccc}
+ & + & + & + & \ldots & + & + & i_{h_{k_{1}}} & + & + & \ldots & + & + & i_{h_{k_{n}}} & + & + & \ldots & + & + \\
+ & + & + & + & \ldots & + & + & i_{h_{k_{1}}} & + & + & \ldots & + & + & i_{h_{k_{n}}} & + & + & \ldots & + & - \\
. & . & \cdot & \cdot & \ldots & . & . & \ldots & . & . & \ldots & . & . & \ldots & . & . & \ldots & . & . \\
+ & - & + & - & \ldots & + & - & i_{h_{k_{1}}} & + & - & \ldots & + & - & i_{h_{k_{n}}} & + & - & \ldots & + & -
\end{array}
$$

as part of the genus- $g$ sector. Again, the matching of spin structures for the genus- $(g-n)$ Ramond sector implies the cancellation of tachyon divergences in this set. When any of the spin structures $i_{h_{k}}$ are replaced by $(++)$, the result remains valid.

Since the modular transformations leave invariant $(++)$, all the spin structures but one will contain a $(++)$ component. This must be matched by a $(+-)$ component, because the tachyon divergence is cancelled by summing over both signs for the B-cycle. Consequently, it is forbidden to use a modular transformation mapping $+-+-\ldots+-$ to $i_{h_{1}} i_{h_{2}} \ldots i_{h_{g}}$ where $i_{h_{n}} \neq(+-)$ for all $n=1, \ldots, g$. Since this restriction applies not only to $+-+-\ldots+-$ but also to the other spin structures in the Ramond sector, $2^{g}$ of the modular transformations constructed using the genus- 1 transformations cannot be used. The remaining $3^{g}-2^{g}-1$ modular transformations $\rho_{r}$ generate the sectors $S_{r}^{\prime}$ correct combinations of spin structures consistent with cancellation of the tachyon divergence.

By the modular transformation of type (iv) in equation (4.5), the spin structure $(+-)$ is interchanged with $(-+)$. While the modular transformations transforming $(+-+-+-\ldots+-)$ to $\left(i_{h_{1}} i_{h_{2}} i_{h_{3}} \ldots i_{h_{g}}\right), i_{h_{n}} \neq(+-)$ are excluded from the set $\left\{\rho_{r}\right\}$, a transformation from $(+-)$ to $(-+)$ for a single handle produces a set of spin structures which still has the property of tachyon divergence cancellation. For example, in the appendix, applying this transformation to the Ramond sector gives the sectors $S_{3}^{\prime}$ and $S_{n_{2}}^{\prime}$ at genus 2 and the sectors $S_{3}^{\prime}, S_{n_{2}}^{\prime}$ and $S_{n_{3}}^{\prime}$ at genus 3 , at arbitrary genus, it would define the sectors $S_{3}^{\prime}, S_{n_{2}}^{\prime}, \ldots, S_{n_{g}}^{\prime}$. Successive applications of these genus-one transformation to different handles will generate another $2^{g}-g-1$ sectors.

Consider the following sum over spin structures

$$
\begin{aligned}
& \sum_{r=1}^{3^{g}-2^{g}+1} \int_{F_{g}} \sum_{(a) \in S_{r}^{\prime}} \eta_{(a)} I_{N, g}=\sum_{\left(a_{N S}\right)=1}^{2^{g}} \eta_{\left(a_{N S}\right)} I_{N, g}^{\left(a_{N S}\right)}+\int_{F_{g}} \sum_{\left(a_{o}\right)=1}^{2^{g-1}} \eta_{\left(a_{o}\right)} I_{N, g}^{\left(a_{o}\right)} \\
& +\int_{F_{g}} \sum_{\left(a_{e}\right)=1}^{2^{g-1}} \eta_{\left(a_{e}\right)} I_{N, g}^{\left(a_{e}\right)}+\sum_{r=3}^{3^{g}-2^{g}+1} \int_{F_{g}} \sum_{\left(a_{o}\right)=1}^{2^{g-1}} \eta_{\rho_{r}\left(a_{o}\right)} I_{N, g}^{\rho_{r}\left(a_{o}\right)} \\
& +\sum_{r=3}^{3^{g}-2^{g}+1} \int_{F_{g}} \sum_{\left(a_{e}\right)=1}^{2^{g-1}} \eta_{\rho_{r}\left(a_{e}\right)} I_{N, g}^{\rho_{r}\left(a_{e}\right)}
\end{aligned}
$$

where $S_{2}^{\prime}=\left\{\left(a_{o}\right)\right\} \cup\left\{\left(a_{e}\right)\right\}$ and $S_{r}^{\prime}=\left\{\rho_{r}\left(a_{o}\right)\right\} \cup\left\{\rho_{r}\left(a_{e}\right)\right\}$, with $\left\{\left(a_{o}\right)\right\}$ and $\left\{\left(a_{e}\right)\right\}$ representing the odd and even spin structures in the Ramond sector. Each of the integrals, labelled by $r$, is free of the infrared divergence in the $\left|K_{n}\right| \rightarrow 0$ limit, since the sum over the $2^{g}$ spin structures in $S_{r}^{\prime}$ removes the tachyon singularity. 
The absence of divergences in the this limit can be used to prove finiteness of the amplitudes in the other degeneration limits. Another divergence associated with the coincidence of fixed points $\xi_{1 n}$ and $\xi_{2 n}$ still arises in the Neveu-Schwarz sector, even after summing over the spin structures, and this infinity can be eliminated only after the Neveu-Schwarz sector is combined with the other sectors. The elimination of divergences arising in the degeneration limit $\left|\xi_{1 n}-\xi_{2 n}\right| \rightarrow 0$ has been demonstrated formally in the proof of finiteness of superstring amplitudes using a manifestly supersymmetric formalism equivalent to the sum over spin structures.

The effect of modular transformations on the Neveu-Schwarz and Ramond sectors is necessary for an estimate of the genus-dependence of the scattering amplitude, as the fermion part of the $g$-loop superstring handle operator ${ }^{29}$ is given by

$$
\begin{aligned}
\langle 0| \Omega_{\phi}^{g-\text { loop }} & =\left(\operatorname{det} C^{g-\text { loop }}\right)^{-5} \cdot\langle 0|: \prod_{i=1}^{5} \Theta\left[\begin{array}{c}
a_{\nu} \\
b_{\nu}
\end{array}\right]\left(\int_{C_{0}} \frac{d z}{2 \pi i} \partial \phi_{i}(z) \cdot \varphi_{\nu}\left(z, z_{0}\right)\right) \\
& \cdot \exp \left\{\frac{1}{2} \sum_{j=1}^{5} \int_{C_{0}} \frac{d x}{2 \pi i} \int_{C_{0}} \frac{d y}{2 \pi i} \partial \phi_{j}(x) \partial \phi_{j}(y)\left[\ln E^{g-l o o p}(x, y)-\ln (x, y)\right]\right\}
\end{aligned}
$$

where $\operatorname{det} C^{g-\text { loop }}=\prod_{\alpha}{ }^{\prime} \prod_{n=1}^{\infty}\left(1-K_{\alpha}^{n}\right), \phi_{i}, i=1, \ldots, \frac{d}{2}$ are bosonized fermions, $\varphi\left(x, x_{0}\right)$ is the abelian integral of first kind, and $E(x, y)$ is the prime form. The presence of the genus-g theta function can be traced to the modular invariance of the string scattering amplitude, since the set of generalized theta functions of rank $r+1-g$ define a basis for complex analytic functions satisfying the transformation rule $f_{i}(t, T z)=\rho_{t}(T) \zeta(T, z)^{r} f_{i}(t, z), T \in S p(2 g ; \mathbb{Z}), z \in \mathbb{Z}, t \in$ $\mathbb{C}^{g} / \mathcal{L}, \mathcal{L} \in(1, \tau) \mathbb{Z}^{2 g}$ with factor of automorphy $\zeta .{ }^{30}$

The fixed-point distance $\left|\xi_{1 n}-\xi_{2 n}\right|$ occurs in the period matrix $\tau_{\mu \nu}$, and the removal of the divergence in the limit $\left|\xi_{1 n}-\xi_{2 n}\right| \rightarrow 0$ may be anticipated from the effect of modular transformations on the theta characteristics $\left[\begin{array}{l}a_{\nu} \\ b_{\nu}\end{array}\right]$.

It is also known that the $g$-loop measure can be expressed as $|F(y, \alpha)|^{2}[\operatorname{sdet}\langle\phi \mid \phi\rangle]^{-5}$ $F(y, \alpha) \sim \frac{1}{y}{ }^{31}$ where $y$ is a coordinate near each boundary of supermoduli space, and $\left\{\phi_{i}\right\}$ are the super-holomorphic half-differentials. The interchangeability of the coordinates in the dependence of $F(y, \alpha)$ near the boundary of supermoduli can viewed to be a consequence of modular invariance. However, the effect of the superdeterminant factor differs for $A$-cycles and dividing cycles.

Consider the fixed-point limit $\left|\xi_{1 n}-\xi_{2 n}\right| \rightarrow 0$ in the sector $S_{r}^{\prime}$. Application of the modular transformation $\rho_{r_{n}}$ interchanges the $A_{n}$-cycle with the $B_{n}$-cycle and the integral becomes

$$
\sum_{(a) \in S_{r}^{\prime}} \int_{F_{g}} \eta_{(a)} I_{N, g}^{(a)}=\sum_{(a) \in \rho_{r_{n}}\left(S_{r}^{\prime}\right)} \int_{\rho_{r_{n}}\left(F_{g}\right)} \eta_{(a)} I_{N, g}^{(a)}
$$

The superstring measure at two and three loops has the property that factors of $\operatorname{det}(C \tau+D)$ in the transformation of the product of theta functions in the integrand are cancelled by the 
transformation of the differential element $\prod_{i \leq j} d \tau_{i j} \wedge d \bar{\tau}_{i j}$. At higher genus, transformation of the theta function ${ }^{32}$,

$$
\begin{aligned}
\vartheta\left[\begin{array}{c}
D n_{1}-C n_{2} \\
-B n_{1}+A n_{2}
\end{array}\right] & \left(0,(A \tau+B)(C \tau+D)^{-1}\right) \\
= & \zeta_{\gamma} \cdot \operatorname{det}(C \tau+D)^{\frac{1}{2}} \\
& \cdot \exp \left(-\pi i^{t} n_{1} \cdot{ }^{t} B D \cdot n_{1}+2 \pi i^{t} n_{1}{ }^{t} B C n_{2}-\pi i t n_{2}{ }^{t} A C n_{2}\right) \vartheta\left[\begin{array}{l}
n_{1} \\
n_{2}
\end{array}\right](0, \tau)
\end{aligned}
$$

differential element, together with a shift in the spin structure. Cancellation of the fixed-point divergence arises because application of modular transformation produces a divergent $\left|K_{n}\right|$ integral with a different spin structure, which can then be cancelled by the divergent part of a multiplier integral of a corresponding spin structure in the same sector. This cancellation may take place in a different integration domain, leaving a finite part with an upper bound which is less than product of the bound in the original fundamental domain and an exponential function of the genus.

The removal of this divergence in $N$-point superstring amplitudes can be verified in the super-Schottky parameterization. The contribution to an N-point g-loop scattering amplitude from this region of supermoduli space is proportional to

$$
\begin{aligned}
& \int_{\Sigma} d^{2} z_{1} \ldots d^{2} z_{N} \sqrt{g\left(z_{1}\right)} \ldots \sqrt{g\left(z_{N}\right)} \\
& \int \frac{d\left(\operatorname{Re} \xi_{1 n}\right) d\left(\operatorname{Re} \xi_{2 n}\right) d\left(\operatorname{Im} \xi_{1 n}\right) d\left(\operatorname{Im} \xi_{2 n}\right) d \theta_{1 n} d \theta_{2 n} d \bar{\theta}_{1 n} d \bar{\theta}_{2 n}}{\left|\xi_{1 n}-\xi_{2 n}-\theta_{1 n} \theta_{2 n}\right|^{2}} \\
& A_{N_{1}+1, n-1} A_{N_{2}+1, g-n} \bar{A}_{2,1}
\end{aligned}
$$

where $A_{N_{1}+1, n-1}$ is the $(n-1)$-loop $N_{1}+1$-point amplitude and $A_{N_{2}+1, g-n}$ is $N_{2}+1$-point genus- $(g-n)$ amplitude, $N_{1}+N_{2}=N$, and $\frac{1}{\left|\xi_{1 n}-\xi_{2 n}-\theta_{1 n} \theta_{2 n}\right|^{2}} \bar{A}_{2,1}^{(a)}=\frac{1}{\left|\xi_{1 n}-\xi_{2 n}-\theta_{1 n} \theta_{2 n}\right|}$

$\hat{A}_{2,1}^{(a)}$ is the two-point one-loop amplitude. In the super-Schottky parameterization, the cancellation of the divergences in the limit $\left|Z_{1 n}-Z_{2 n}\right| \rightarrow 0$ can be deduced after summing over $2^{2 g}$ genus-g spin structures. Splitting the spin structures into a genus- $(n-1)$ component genus$(g-n)$ component and a handle with a degenerating $B$-cycle, it follows that the vanishing of the sum $\sum_{(a)=1}^{2^{2 g}} A_{N_{1}+1, n-1}^{(a)} A_{N_{2}+1, g-n}^{(a)} A_{2,1}^{(a)}=\sum_{\left(a_{n-1}\right)=1}^{2^{n-1}} A_{N_{1}+1, n-1}^{\left(a_{n-1}\right)}$ $\sum_{\left(a_{g-n}\right)=1}^{2^{g-n}} A_{N_{2}+1, g-n}^{\left(a_{g-n}\right)} \sum_{\left(a_{1}\right)=1}^{4} A_{N_{3}+2,1}^{\left(a_{1}\right)}$ as $\left|\xi_{1 n}-\xi_{2 n}\right| \rightarrow 0$ removes the divergence in the integral (4.15) arising from this limit.

In the limit $Z_{1 n}-Z_{2 n} \rightarrow 0$, the integrand has the form

$$
\begin{aligned}
\sum_{\left(a_{n-1}\right)=1}^{2^{n-1}} A_{N_{1}+1, n-1}^{\left(a_{n-1}\right)} \sum_{\left(a_{g-n}\right)=1}^{2^{g-n}} A_{N_{2}+1, g-n}^{\left(a_{g-n}\right)} & \lim _{Z_{1 n} \rightarrow Z_{2 n}}\left[\frac{1}{\left|\xi_{1 n}-\xi_{2 n}-\theta_{1 n} \theta_{2 n}\right|} \sum_{\left(a_{1}\right)=1}^{4} \hat{A}_{2,1}^{\left(a_{1}\right)}\right. \\
& \left.+\sum_{\left(a_{1}\right)=1}^{4} \frac{d}{d\left|\xi_{1 n}-\xi_{2 n}-\theta_{1 n} \theta_{2 n}\right|} \hat{A}_{2,1}^{\left(a_{1}\right)}+\ldots\right]
\end{aligned}
$$


The choice of the expression $\hat{A}_{2,1}^{(a)}$ follows from the the one-loop closed superstring amplitude before the summation of spin structures, which contains a $\frac{1}{|y|}$ singularity ${ }^{30}$ near the boundary of moduli space $|y|=0$ that can be be obtained through the degeneration of the either homology cycle. The degeneration of the $A$-cycle given by $|K| \rightarrow 0$ can be transformed to the degeneration of the $B$-cycle $|K| \rightarrow 1$ under a modular transformation, and this further is equivalent to the coalescence of the fixed points since $|1-K|=|\gamma||K|^{\frac{1}{2}}\left|\xi_{1}-\xi_{2}\right|$. It also can be deduced from the N-point one-loop open superstring amplitude ${ }^{33}$

$$
A_{N, 1}^{\text {open }}=C \int_{0}^{1} \prod_{i=1}^{N-1} \theta\left(\nu_{I+1}-\nu_{I}\right) d \nu_{I} \int_{0}^{1} \frac{d q}{q} \prod_{I<J}\left(\psi_{I J}\right)^{k_{i} \cdot k_{j}}
$$

so that the N-point closed superstring amplitude includes the integral $\int \frac{d q \wedge d \bar{q}}{\mid q q^{2}}$. Fixing the radius of the isometric circles in the Schottky uniformization of the surface, so that $|\gamma|$ is constant, the derivative of the one-loop closed superstring integral with respect to the distance between the fixed points equals is $-\frac{1}{2}|\gamma|^{-1}|K|^{-\frac{3}{2}}(1+|K|) \frac{d}{d|K|} \hat{A}_{2,1}=\frac{1}{4 \pi}|\gamma|^{-1}|K|^{-\frac{5}{2}}(1+|K|) \frac{d}{d \operatorname{Im} \tau} \hat{A}_{2,1}$.

With the genus-two period matrix $\Omega=\left(\begin{array}{cc}\tau_{1} & \tau \\ \tau & \tau_{1}\end{array}\right)$ and the genus-one spin structures $\left[\mu_{1}\right]=$ $\left[\begin{array}{ll}0 & 0\end{array}\right],\left[\mu_{3}\right]=\left[\begin{array}{ll}0 & \frac{1}{2}\end{array}\right],\left[\mu_{5}\right]=\left[\begin{array}{ll}\frac{1}{2} & 0\end{array}\right],\left[\mu_{2}\right]=\left[\begin{array}{ll}0 & 0\end{array}\right],\left[\mu_{4}\right]=\left[\begin{array}{ll}0 & \frac{1}{2}\end{array}\right],\left[\mu_{6}\right]=\left[\begin{array}{ll}\frac{1}{2} & 0\end{array}\right],\left[\nu_{0}\right]=\left[\begin{array}{ll}\frac{1}{2} & \frac{1}{2}\end{array}\right]$, the asymptotic behavour of the chiral superstring measure ${ }^{34}$ is given by

$$
\begin{aligned}
& d \mu\left[\begin{array}{l}
\mu_{1} \\
\mu_{2}
\end{array}\right](\Omega)=\frac{1}{2^{10} \pi^{8} \tau^{2}}\left\langle\mu_{1} \mid \nu_{0}\right\rangle\left\langle\mu_{2} \mid \nu_{0}\right\rangle \frac{\vartheta_{1}\left[\mu_{1}\right]^{4}\left(\tau_{1}\right) \vartheta_{1}\left[\mu_{2}\right]^{4}\left(\tau_{2}\right)}{\eta\left(\tau_{1}\right)^{12} \eta\left(\tau_{2}\right)^{12}} d \tau_{1} d \tau_{2} d \tau+\mathcal{O}\left(\tau^{0}\right) \\
& d \mu\left[\begin{array}{l}
\nu_{0} \\
\nu_{0}
\end{array}\right](\Omega)=\frac{3 \tau^{2}}{2^{6} \pi^{4}} d \tau_{1} d \tau_{2} d \tau+\mathcal{O}\left(\tau^{4}\right)
\end{aligned}
$$

for separating degenerations and 


$$
\begin{aligned}
& d \mu\left[\begin{array}{l}
\mu_{1} \\
\mu_{2}
\end{array}\right]=-d \mu\left[\begin{array}{l}
\mu_{1} \\
\mu_{4}
\end{array}\right]=\frac{\vartheta_{1}\left[\mu_{1}\right]\left(\frac{\tau}{2}, \tau_{1}\right)^{4}+\vartheta_{1}\left[\nu_{0}\right]\left(\frac{\tau}{2}, \tau_{1}\right)^{4}}{2^{12} \pi^{6} \cdot q \eta\left(\tau_{1}\right)^{18} \vartheta_{1}\left[\nu_{0}\right]\left(\frac{\tau}{2}, \tau_{1}\right)^{2}} \\
& \vartheta_{1}\left[\mu_{1}\right]\left(0, \tau_{1}\right)^{4} \vartheta_{1}\left[\mu_{3}\right]\left(0, \tau_{1}\right)^{4} \vartheta_{1}\left[\mu_{5}\right]\left(0, \tau_{1}\right)^{4} d \tau_{1} d \tau_{2} d \tau+ \\
& -\frac{1}{2^{12} \pi^{6} \cdot \eta\left(\tau_{1}\right)^{18} \vartheta_{1}\left[\nu_{0}\right]\left(\frac{\tau}{2}, \tau_{1}\right)^{2}}\left\{4 \left(\vartheta_{1}\left[\mu_{3}\right](0, \tau)^{4} \vartheta_{1}\left[\mu_{5}\right]\left(0, \tau_{1}\right)^{3}\right.\right. \\
& \text { - } \left.\vartheta_{1}\left[\mu_{5}\right]\left(\tau, \tau_{1}\right)+\vartheta_{1}\left[\mu_{3}\right]\left(0, \tau_{1}\right)^{3} \vartheta_{1}\left[\mu_{5}\right](0, \tau)^{4} \vartheta_{1}\left[\mu_{3}\right]\left(\tau, \tau_{1}\right)^{4}\right) \\
& \vartheta_{1}\left[\mu_{3}\right]\left(\frac{\tau}{2}, \tau_{1}\right)^{4} \\
& +4\left(-\vartheta_{1}\left[\mu_{3}\right]\left(0, \tau_{1}\right)^{3} \vartheta_{1}\left[\mu_{5}\right]\left(0, \tau_{1}\right)^{4} \vartheta_{1}\left[\mu_{3}\right]\left(\tau, \tau_{1}\right)+\vartheta_{1}\left[\mu_{3}\right](0, \tau)^{4}\right. \\
& \left.\left.\vartheta_{1}\left[\mu_{5}\right]\left(0, \tau_{1}\right)^{3} \vartheta_{1}\left[\mu_{5}\right]\left(\tau, \tau_{1}\right)\right) \vartheta_{1}\left[\mu_{5}\right]\left(\frac{\tau}{2}, \tau_{1}\right)^{4}\right) \\
& +16 \vartheta_{1}\left[\nu_{0}\right]\left(\frac{\tau}{2}, \tau_{1}\right)^{4} \vartheta_{1}\left[\mu_{1}\right](0, \tau)^{4} \vartheta_{1}\left[\mu_{1}\right]\left(\frac{\tau}{2}, \tau_{1}\right)^{4} \\
& +4 \vartheta_{1}\left[\mu_{1}\right]\left(0, \tau_{1}\right)^{3} \vartheta_{1}\left[\mu_{1}\right]\left(\tau, \tau_{1}\right) \frac{\vartheta_{1}\left[\mu_{1}\right]\left(\frac{\tau}{2}, \tau_{1}\right)^{4}+\vartheta_{1}\left[\nu_{0}\right]\left(\frac{\tau}{2}, \tau_{1}\right)^{4}}{2^{12} \pi^{6} \cdot \eta\left(\tau_{1}\right)^{18} \vartheta_{1}\left[\nu_{0}\right]\left(\frac{\tau}{2}, \tau_{1}\right)^{2}} \\
& \left.\vartheta_{1}\left[\mu_{1}\right]\left(0, \tau_{1}\right)^{4} \vartheta_{1}\left[\mu_{3}\right]\left(0, \tau_{1}\right)^{4} \vartheta_{1}\left[\mu_{5}\right]\left(0, \tau_{1}\right)^{4}\right\} d \tau_{1} d \tau_{2} d \tau+\mathcal{O}\left(q^{0}\right) \\
& d \mu\left[\begin{array}{l}
\mu_{3} \\
\mu_{2}
\end{array}\right]=-d \mu\left[\begin{array}{l}
\mu_{3} \\
\mu_{4}
\end{array}\right]=\frac{-\vartheta_{1}\left[\mu_{3}\right]\left(\frac{\tau}{2}, \tau_{1}\right)^{4}+\vartheta_{1}\left[\nu_{0}\right]\left(\frac{\tau}{2}, \tau_{1}\right)^{4}}{2^{12} \pi^{6} \cdot q \eta\left(\tau_{1}\right)^{18} \vartheta_{1}\left[\nu_{0}\right]\left(\frac{\tau}{2}, \tau_{1}\right)^{2}} \\
& \vartheta_{1}\left[\mu_{1}\right]\left(0, \tau_{1}\right)^{4} \vartheta_{1}\left[\mu_{3}\right]\left(0, \tau_{1}\right)^{4} \vartheta_{1}\left[\mu_{5}\right]\left(0, \tau_{1}\right)^{4} d \tau_{1} d \tau_{2} d \tau \\
& +\frac{1}{2^{12} \pi^{6} \cdot \eta\left(\tau_{1}\right)^{18} \vartheta_{1}\left[\nu_{0}\right]\left(\frac{\tau}{2}, \tau_{1}\right)^{2}} \\
& \left\{4 \vartheta _ { 1 } [ \mu _ { 1 } ] ( \frac { \tau } { 2 } , \tau _ { 1 } ) ^ { 4 } \left(\vartheta_{1}\left[\mu_{3}\right]\left(0, \tau_{1}\right)^{3} \vartheta_{1}\left[\mu_{3}\right]\left(\tau, \tau_{1}\right) \cdot \vartheta_{1}\left[\mu_{1}\right]\left(0, \tau_{1}\right)^{4}\right.\right. \\
& \left.-\vartheta_{1}\left[\mu_{1}\right]\left(0, \tau_{1}\right)^{3} \vartheta\left[\mu_{1}\right]\left(\tau, \tau_{1}\right) \vartheta_{1}\left[\mu_{3}\right]\left(0, \tau_{1}\right)^{4}\right) \\
& +16 \vartheta_{1}\left[\nu_{0}\right]\left(\frac{\tau}{2}, \tau_{1}\right)^{4} \vartheta_{1}\left[\mu_{3}\right]\left(\frac{\tau}{2}, \tau_{1}\right)^{4} \cdot \vartheta_{1}\left[\mu_{3}\right]\left(0, \tau_{1}\right)^{4} \\
& -4 \vartheta_{1}\left[\mu_{5}\right]\left(\frac{\tau}{2}, \tau_{1}\right)^{4}\left(\vartheta_{1}\left[\mu_{1}\right]\left(0, \tau_{1}\right)^{3} \vartheta_{1}\left[\mu_{1}\right]\left(\tau, \tau_{1}\right) \vartheta_{1}\left[\mu_{5}\right]\left(0, \tau_{1}\right)^{4}\right. \\
& \left.+\vartheta_{1}\left[\mu_{5}\right]\left(0, \tau_{1}\right)^{3} \vartheta_{1}\left[\mu_{5}\right]\left(\tau, \tau_{1}\right) \vartheta_{1}\left[\mu_{1}\right]\left(0, \tau_{1}\right)\right) \\
& +4 \vartheta_{1}\left[\mu_{3}\right]\left(0, \tau_{1}\right) \vartheta\left[\mu_{3}\right]\left(\tau, \tau_{1}\right) \frac{-\vartheta_{1}\left[\mu_{3}\right]\left(\frac{\tau}{2}, \tau_{1}\right)^{4}+\vartheta_{1}\left[\nu_{0}\right]\left(\frac{\tau}{2}, \tau_{1}\right)^{4}}{2^{12} \pi^{6} \cdot \eta\left(\tau_{1}\right)^{18} \vartheta_{1}\left[\nu_{0}\right]\left(\frac{\tau}{2}, \tau_{1}\right)^{2}} \\
& \left.\vartheta_{1}\left[\mu_{1}\right]\left(0, \tau_{1}\right)^{4} \vartheta_{1}\left[\mu_{3}\right]\left(0, \tau_{1}\right)^{4} \vartheta_{1}\left[\mu_{5}\right]\left(0, \tau_{1}\right)^{4}\right\} d \tau_{1} d \tau_{2} d \tau+\mathcal{O}\left(q^{0}\right)
\end{aligned}
$$




$$
\begin{aligned}
& d \mu\left[\begin{array}{l}
\mu_{5} \\
\mu_{2}
\end{array}\right]=-d \mu\left[\begin{array}{l}
\mu_{5} \\
\mu_{4}
\end{array}\right]=\frac{-\vartheta_{1}\left[\mu_{5}\right]\left(\frac{\tau}{2}, \tau_{1}\right)^{4}+\vartheta_{1}\left[\nu_{0}\right]\left(\frac{\tau}{2}, \tau_{1}\right)^{4}}{2^{12} \pi^{6} \cdot q \eta\left(\tau_{1}\right)^{18} \vartheta_{1}\left[\nu_{0}\right]\left(\frac{\tau}{2}, \tau_{1}\right)^{2}} \\
& \vartheta_{1}\left[\mu_{1}\right]\left(0, \tau_{1}\right)^{4} \vartheta_{1}\left[\mu_{3}\right]\left(0, \tau_{1}\right)^{4} \vartheta_{1}\left[\mu_{5}\right]\left(0, \tau_{1}\right)^{4} d \tau_{1} d \tau_{2} d \tau \\
& -\frac{1}{2^{12} \pi^{6} \cdot \eta\left(\tau_{1}\right)^{18} \vartheta_{1}\left[\nu_{0}\right]\left(\frac{\tau}{2}, \tau_{1}\right)^{2}}\left\{4 \left(\vartheta_{1}\left[\mu_{3}\right]\left(0, \tau_{1}\right)^{3} \vartheta_{1}\left[\mu_{3}\right]\left(\tau, \tau_{1}\right)\right.\right. \\
& \vartheta\left[\mu_{1}\right]\left(0, \tau_{1}\right)^{4}-\vartheta_{1}\left[\mu_{3}\right]\left(0, \tau_{1}\right)^{4} \\
& \left.\vartheta_{1}\left[\mu_{1}\right](0, \tau)^{3} \vartheta_{1}\left[\mu_{3}\right]\left(\tau, \tau_{1}\right)\right)\left(\vartheta_{1}\left[\mu_{1}\right]\left(\frac{\tau}{2}, \tau_{1}\right)^{4}+\vartheta_{1}\left[\mu_{3}\right]\left(\frac{\tau}{2}, \tau_{1}\right)^{4}\right) \\
& +16 \vartheta_{1}\left[\nu_{0}\right]\left(\frac{\tau}{2}, \tau_{1}\right)^{4} \vartheta_{1}\left[\mu_{3}\right](0, \tau)^{4} \vartheta_{1}\left[\mu_{5}\right]\left(\frac{\tau}{2}, \tau_{1}\right) \\
& +4 \vartheta_{1}\left[\mu_{5}\right]\left(0, \tau_{1}\right)^{3} \vartheta_{1}\left[\mu_{5}\right]\left(\tau, \tau_{1}\right) \frac{-\vartheta_{1}\left[\mu_{5}\right]\left(\frac{\tau}{2}, \tau_{1}\right)^{4}+\vartheta_{1}\left[\nu_{0}\right]\left(\frac{\tau}{2}, \tau_{1}\right)^{4}}{2^{12} \pi^{6} \cdot \eta\left(\tau_{1}\right)^{18} \vartheta_{1}\left[\nu_{0}\right]\left(\frac{\tau}{2}, \tau_{1}\right)^{2}} \\
& \left.\vartheta_{1}\left[\mu_{1}\right]\left(0, \tau_{1}\right)^{4} \vartheta_{1}\left[\mu_{3}\right]\left(0, \tau_{1}\right)^{4} \vartheta_{1}\left[\mu_{5}\right]\left(0, \tau_{1}\right)^{4}\right\} d \tau_{1} d \tau_{2} d \tau+\mathcal{O}\left(q^{0}\right) \\
& d \mu\left[\begin{array}{l}
\nu_{0} \\
\nu_{0}
\end{array}\right]=\vartheta_{1}\left[\nu_{0}\right]\left(\frac{\tau}{2}, \tau_{1}\right) \sum_{i=1,3,5} \frac{\left\langle\mu_{i} \mid \nu_{0}\right\rangle \vartheta_{1}\left[\mu_{i}\right]\left(0, \tau_{1}\right)^{8} \vartheta_{1}\left[\mu_{i}\right]\left(\frac{\tau}{2}, \tau_{1}\right)^{4}}{256 \pi^{6} \eta\left(\tau_{1}\right)^{18} \vartheta_{1}\left[\nu_{0}\right]\left(\frac{\tau}{2}, \tau_{1}\right)^{2}}+\mathcal{O}(q) \\
& d \mu\left[\begin{array}{l}
\mu_{1} \\
\mu_{6}
\end{array}\right]=-\frac{\vartheta_{1}\left[\mu_{2}\right]\left(\frac{\tau}{2}, \tau_{1}\right)^{4}}{2^{9} \pi^{6} \eta\left(\tau_{1}\right)^{18} \vartheta_{1}\left[\nu_{0}\right]\left(\frac{\tau}{2}, \tau_{1}\right)^{2}}\left[2 \vartheta_{1}\left[\mu_{1}\right]\left(0, \tau_{1}\right)^{4} \vartheta_{1}\left[\mu_{3}\right]\left(0, \tau_{1}\right)^{4} \vartheta_{1}\left[\mu_{3}\right]\left(\frac{\tau}{2}, \tau_{1}\right)^{4}\right. \\
& +\vartheta_{1}\left[\mu_{5}\right]\left(0, \tau_{1}\right)^{3} \vartheta_{1}\left[\mu_{5}\right]\left(\tau, \tau_{1}\right) \vartheta_{1}\left[\mu_{3}\right]\left(0, \tau_{1}\right)^{4} \vartheta_{1}\left[\mu_{1}\right]\left(0, \tau_{1}\right)^{4} \\
& +\vartheta_{1}\left[\mu_{3}\right]\left(0, \tau_{1}\right)^{3} \vartheta_{1}\left[\mu_{3}\right]\left(\tau, \tau_{1}\right) \vartheta_{1}\left[\mu_{5}\right]\left(0, \tau_{1}\right)^{4} \vartheta_{1}\left[\mu_{1}\right]\left(0, \tau_{1}\right)^{4} \\
& \left.-\vartheta_{1}\left[\mu_{1}\right]\left(0, \tau_{1}\right)^{3} \vartheta_{1}\left[\mu_{1}\right]\left(\tau, \tau_{1}\right) \vartheta_{1}\left[\mu_{3}\right]\left(0, \tau_{1}\right)^{4} \vartheta_{1}\left[\mu_{5}\right]\left(0, \tau_{1}\right)^{4}\right] \\
& +\mathcal{O}(q) \\
& d \mu\left[\begin{array}{l}
\mu_{3} \\
\mu_{6}
\end{array}\right]=-\frac{\vartheta_{1}\left[\mu_{4}\right]\left(\frac{\tau}{2}, \tau_{1}\right)^{4}}{2^{8} \pi^{6} \eta\left(\tau_{1}\right) \vartheta_{1}\left[\nu_{0}\right]\left(\frac{\tau}{2}, \tau_{1}\right)^{2}}\left[\vartheta_{1}\left[\mu_{3}\right]\left(0, \tau_{1}\right)^{8} \vartheta_{1}\left[\nu_{0}\right]\left(\frac{\tau}{2}, \tau_{1}\right)^{4}\right. \\
& \left.-\vartheta_{1}\left[\mu_{1}\right]\left(0, \tau_{1}\right)^{8} \vartheta_{1}\left[\mu_{5}\right]\left(\frac{\tau}{2}, \tau_{1}\right)^{4}+\vartheta_{1}\left[\mu_{5}\right]\left(0, \tau_{1}\right)^{8} \vartheta\left[\mu_{1}\right]\left(\frac{\tau}{2}, \tau_{1}\right)^{4}\right]+\mathcal{O}(q) \\
& d \mu\left[\begin{array}{l}
\mu_{5} \\
\mu_{6}
\end{array}\right]=-\frac{\vartheta_{1}\left[\mu_{6}\right]\left(\frac{\tau}{2}, \tau_{1}\right)^{4}}{2^{8} \pi^{6} \eta\left(\tau_{1}\right)^{18} \vartheta_{1}\left[\nu_{0}\right]\left(\frac{\tau}{2}, \tau_{1}\right)^{2}}\left[\vartheta_{1}\left[\mu_{5}\right]\left(0, \tau_{1}\right)^{8} \vartheta_{1}\left[\nu_{0}\right]\left(\frac{\tau}{2}, \tau_{1}\right)^{4}\right. \\
& \left.+\vartheta_{1}\left[\mu_{1}\right]\left(0, \tau_{1}\right)^{8} \vartheta_{1}\left[\mu_{3}\right]\left(\frac{\tau}{2}, \tau_{1}\right)^{4}+\vartheta_{1}\left[\mu_{3}\right]\left(0, \tau_{1}\right)^{8} \vartheta_{1}\left[\mu_{1}\right]\left(\frac{\tau}{2}, \tau_{1}\right)^{4}\right]+\mathcal{O}(q)
\end{aligned}
$$

for non-separating divergences, with the paramter $q$ determining the size of the degenerating cycle. Summing over the spin structures for a genus-one component of the Riemann surface eliminates the $\frac{1}{q}$ tachyon divergence. The finite part of the integrand of order $q^{0}$ defines the genus-independent contribution to the superstring amplitude, which can be bounded by $B_{B}$. The dominant contribution in the fixed-point distance limit is obtained from the simultaneous degeneration of $B_{m}$-cycles, $m=2, \ldots, g-1$, which would be bounded by $B_{B}^{g-2}$. The use of 
a genus-2 component facilitates the analysis of the fixed-point distance limit, with the result that the genus-independence of the finite part of the superstring amplitude in the limit of a vanishing $B$-cycle in any single handle can be verified.

The string amplitude at $g$ loops contains a genus-g theta function that would tend to an expression containing a product of genus- 2 theta functions in the degeneration limit with genus- 2 components. Each of the three moduli parameters for a genus- 2 component define the different degeneration limits, and the sum over all such boundaries in the genus-g moduli space can be obtained by overlapping these three limits. Dividing a genus-g surface into genus- 2 components and considering the degeneration limits of these components in the even-spin structure sector, the genus-dependence of a contribution to the superstring amplitude can be estimated by determining the next-order term in the expansion of the the superstring measure, summed over the different spin structures. To obtain the contribution of overlapping components, the factorization of a genus- 3 amplitude $A_{3}$ in the degeneration limit $2 A_{1} A_{2}+A_{1} A_{1} A_{1}+3 B_{2}+2 C_{2}+3 D_{1}+D_{1}^{\prime}$ can be used, where $A_{i}$ defines the amplitude of the genus-i component obtained by factorizing along a dividing cycle, while $B_{2}, C_{2}$ denote the amplitudes defined by the punctured genus- 2 surfaces, and $D_{1}, D_{1}^{\prime}$ are amplitudes for punctured genus-1 surfaces. The divergences will be cancelled in the summation over the spin structures for the genus- 1 and genus- 2 components separately, and the product of the next-order terms for each component will define the leading contribution to this amplitude. At genus $g$, there are three additional modular parameters and $g_{1} g_{2}$ extra period matrix elements arising from the factorization of a genus-g amplitude into a genus- $g_{1}$ and genus- $g_{2}$ component. The genus-dependence of the superstring amplitude also could be determined by the leading order contribution to the genus- $g$ theta function after elimination of the sums with non-derivative terms.

While the limit $\left|B_{m}\right| \rightarrow 0$ implies that $\left|\xi_{2 j}\right| \rightarrow 0, j=m, \ldots, g$, factors of $\left|\xi_{2 m}\right|$ are cancelled in the integrand. Using the expression for $H_{n}$, it follows that

$$
\begin{gathered}
\frac{d H_{n} \wedge d \bar{H}_{n}}{\left|H_{n}\right|^{3}} \sim \frac{\left|\xi_{2 n}\right|^{2} d^{2} \xi_{1 n}-\xi_{1 n} \bar{\xi}_{2 n} d \xi_{2 n} \wedge d \bar{\xi}_{1 n}-\xi_{2 n} \bar{\xi}_{1 n} d \xi_{1 n} \wedge d \bar{\xi}_{2 n}+\left|\xi_{1 n}\right|^{2} d^{2} \xi_{2 n}}{\left|\xi_{1 n}\right|\left|\xi_{1 n}-\xi_{2 n}\right|^{3}}+ \\
+\frac{3}{4}\left|\frac{\xi_{1 n}}{\xi_{1 n}-\xi_{2 n}}\right|^{3} \vartheta_{1 n} \vartheta_{2 n} \bar{\vartheta}_{1 n} \bar{\vartheta}_{2 n} d\left|H_{n}\right| d \theta_{n}^{H}+\ldots
\end{gathered}
$$

whereas

$$
\prod_{m=2}^{g} \frac{d \xi_{2 m} \wedge d \bar{\xi}_{2 m}}{\left|\xi_{2 m}\right|^{2}}=\prod_{j=2}^{g} \frac{d B_{j} \wedge d \bar{B}_{j}}{\left|B_{j}\right|^{2}}
$$

The remaining factor of $\left|B_{j}\right|$ gives rise to an integral of the form $\int \frac{d B_{j} \wedge d \bar{B}_{j}}{\left|B_{j}\right|}$ which is not divergent. The extra factor of $\left|\xi_{1 n}\right|$ is also cancelled by $d^{2} \xi_{1 n}$ so that the only fixed-point divergence is obtained in the limit $\left|\xi_{1 n}-\xi_{2 n}\right| \rightarrow 0$. Fixing the location of $\xi_{1 g}$ to be 0 , the expression $\left|\xi_{2 g}\right|$ is obtained in the denominator, with the identical power as the fixed point distance $\left|\xi_{1 n}-\xi_{2 n}\right|$. As the vanishing of $\left|B_{j}\right|, j=2, \ldots g$ implies that $\left|\xi_{2 g}\right| \rightarrow 0$, the expansion in powers of $\left|\xi_{2 g}\right|$ again leads to a remainder term containing the derivative $\frac{d}{d\left|\xi_{2 g}\right|} \hat{A}_{2,1}$ which is independent of the genus. 
After summation over spin structures, the product of the integrals over $H_{m}, m=2, \ldots, g-1$ and $B_{m}, m=2, \ldots, g$ would increase at most exponentially with respect to the genus.

The measure associated with even spin structures in the Neveu-Schwarz-Ramond sector has been obtained by solving Ward identities and using vacuum correlation functions for the different spin structures. ${ }^{16,36,37}$ The spin structures can be labelled as $L=\sum_{n}\left(l_{1 n}, l_{2 n}\right)$, $l_{i n}=\left(0, \frac{1}{2}\right)$, so that they are even or odd if $4 \sum_{n=1}^{g} l_{1 n} l_{2 n}$ is even or odd respectively. ${ }^{14}$

The coordinates of a super-Riemann surface change under $2 \pi$-twists around $A_{n}$-cycles and traversals of $B_{n}$-cycles as

$$
\begin{aligned}
& \Gamma_{A_{n}}^{(0)}\left(l_{1 n}\right)=\left\{z \rightarrow z, \theta \rightarrow(-1)^{2 l_{1 n}} \theta\right\} \\
& \Gamma_{B_{n}}^{(0)}\left(l_{2 n}\right)=\left\{z \rightarrow T_{n} z, \theta \rightarrow \frac{(-1)^{2 l_{2 n}} \theta}{\gamma_{n} z+\delta_{n}}\right\}
\end{aligned}
$$

when the odd modular parameters are zero. ${ }^{16}$ Thus, the sign of the fermionic coordinate is unchanged by a circuit around an $A_{n}$-cycle if $l_{1 n}=0$ and is multiplied by -1 when $l_{1 n}=\frac{1}{2}$; similarly, $l_{2 n}=0$ is associated with a plus sign, while $l_{2 n}=\frac{1}{2}$ corresponds to a negative sign.

Considering the map from the parallelogram to the annulus, the target space coordinate in $X=e^{2 \pi i Z}=x+\theta \psi$ is unchanged by $Z \rightarrow Z+1$, but the fermionic coordinates change sign $\theta \rightarrow e^{\pi i} \theta$ and $\psi \rightarrow e^{\pi i} \psi$. Since the annulus can be mapped to the Schottky plane with two disks removed without changing the sign of the fermions the sign of the fermionic coordinate, the following nomenclature can be established for genus-one surfaces:

$$
\begin{aligned}
(0,0) & \leftrightarrow(+,+)_{\text {Sch. }} \leftrightarrow(-,-)_{\text {par. }} \\
\left(\frac{1}{2}, 0\right) & \leftrightarrow(-,+)_{\text {Sch. }} \leftrightarrow(+,-)_{\text {par. }} \\
\left(0, \frac{1}{2}\right) & \leftrightarrow(+,-)_{\text {Sch. }} \leftrightarrow(-,+)_{\text {par. }} \\
\left(\frac{1}{2}, \frac{1}{2}\right) & \leftrightarrow(-,-)_{S c h .} \leftrightarrow(+,+)_{\text {par. }}
\end{aligned}
$$

With this labelling of the spin structures, the integrand in the superstring partition function for spin structures in the Neveu-Schwarz-Ramond sectors is given by

$$
\begin{gathered}
Z_{L, L^{\prime}}^{(g)}=\kappa^{2 g}\left[\operatorname{det}\left(\overline{\mathcal{T}}\left(\left\{K_{n}, Z_{1 n}, Z_{2 n}\right\}, L^{\prime}\right)-\mathcal{T}\left(\left\{K_{n}, Z_{1 n}, Z_{2 n}\right\}, L\right)\right]^{-5}\right. \\
Z_{L}^{(g)}\left(\left\{K_{n}, Z_{1 n}, Z_{2 n}\right\}\right) \bar{Z}_{L^{\prime}}^{(g)}\left(\left\{K_{n}, Z_{1 n}, Z_{2 n}\right\}\right)
\end{gathered}
$$

where $\mathcal{T}\left(\left\{K_{n}, Z_{1 n}, Z_{2 n}\right\}\right)$ is the super-period matrix $Z_{L}^{(g)}\left(\left\{K_{n}, Z_{1 n}, Z_{2 n}\right\}\right)$ is a holomorphic 
function of the super-Schottky parameters.

$$
\begin{aligned}
Z_{L}\left(\left\{K_{n}, Z_{1 n}, Z_{2 n}\right\}\right)= & Z_{0(m)}\left(\left\{K_{n}, \xi_{1 n}, \xi_{2 n}\right\}, L\right) Z_{0(g h)}\left(\left\{K_{n}, \xi_{1 n}, \xi_{2 n}\right\}, L\right) \\
& \cdot \Upsilon_{m}^{(g)}\left(\left\{K_{n}, Z_{1 n}, Z_{2 n}\right\}, L\right) \Upsilon_{g h}^{(g)}\left(\left\{K_{n}, Z_{1 n}, Z_{2 n}\right\}, L\right) \\
& \cdot H\left(\left\{K_{n}, Z_{1 n}, Z_{2 n}\right\}\right) \\
H\left(\left\{K_{n}, Z_{1 n}, Z_{2 n}\right\}\right)= & \left(u_{1}-u_{2}\right)\left(v_{1}-u_{2}\right)\left[1-\frac{\mu_{1} \mu_{2}}{2\left(u_{1}-u_{2}\right)}-\frac{\nu_{1} \mu_{2}}{2\left(v_{1}-u_{2}\right)}\right] \\
& \prod_{n=1}^{g}\left(Z_{1 n}-Z_{2 n}\right)^{-1}
\end{aligned}
$$

where $Z_{0(m)}$ and $Z_{0(g h)}$ represent string superfield and ghost contributions evaluated at zero odd Schottky parameters, $\ln \Upsilon_{m}^{(g)}\left(\left\{K_{n}, Z_{1 n}, Z_{2 n}\right\}, L\right)$ and $\ln \Upsilon_{g h}^{(g)}\left(\left\{K_{n}, Z_{1 n}, Z_{2 n}\right\}, L\right)$ contain terms proportional to odd Schottky parameters, and $u_{1}, v_{1}, u_{2}, \mu_{1}, \nu_{1}, \mu_{2}$ are fixed for every genus- $g$ super-Riemann surface and therefore are not moduli. ${ }^{3}$ Integrating over the supermoduli and summing over the spin structures $\left(L, L^{\prime}\right)$ gives the total partition function. Supermodular invariance of this partition function has been verified formally using Ward identities and established explicitly for specific transformations between spin structures of genus-2 components of arbitrary-genus Riemann surfaces. ${ }^{37}$

Expressions for $Z_{0(m)}$ and $Z_{0(g h)}$ bear a resemblance to the formulae for the Neveu-Schwarz sector. ${ }^{16}$

$$
\begin{aligned}
& Z_{0(m)}\left(\left\{K_{n}, \xi_{1 n}, \xi_{2 n}, L\right)\right.=\frac{\Theta\left[l_{1}, l_{2}\right]\left(0 \mid \omega^{(r)}\right)}{\Theta^{5}\left[\{0\},\left\{\frac{1}{2}\right\}\right]\left(0 \mid \omega^{(r)}\right)} \prod_{\alpha}^{\prime} \prod_{p=1}^{\infty} \frac{\left(1-K_{\alpha}^{p-\frac{1}{2}}\right)}{\left(1-K_{\alpha}^{p}\right)^{10}} \\
& Z_{0(g h)}\left(\left\{K_{n}, \xi_{1 n}, \xi_{2 n}\right\}, L\right)=\frac{e^{-\pi i \sum_{j, r} l_{1 j} l_{1 r} \tau_{j r}^{(r)}}}{\sqrt{\operatorname{det} \tilde{M}\left(\left\{\sigma_{n}\right\}\right) \operatorname{det} \tilde{M}\left(\left\{-\sigma_{n}\right\}\right)}} \prod_{n=1}^{g} \tilde{Z}_{0}\left(K_{n} ; l_{1 n}, l_{2 n}\right) \\
& \prod_{\alpha}^{\prime} \prod_{p=1}^{\infty} \frac{\left(1-K_{\alpha}^{p+1}\right)^{2}}{\left[1-\Lambda\left(\left\{K_{n}\right\},\left\{\sigma_{n}\right\}\right) K_{\alpha}^{p+\frac{1}{2}}\right]\left[1-\Lambda\left(\left\{K_{n}\right\},\left\{-\sigma_{n}\right\}\right) K_{\alpha}^{p+\frac{1}{2}}\right]}
\end{aligned}
$$

where $\tau_{j r}$ is the value of the super-period matrix $\mathcal{T}_{j r}$ at zero odd parameter, $\tilde{M}\left(\left\{\sigma_{n}\right\}\right)$, is a matrix relating conformal $\frac{3}{2}$-tensors to $\frac{3}{2}$-tensors arising in the transformation rule of spinstructure dependent Green functions, ${ }^{35} \Lambda\left(K_{\alpha},\left\{\sigma_{n}\right\}\right)=e^{\Omega_{V_{\beta}\left(K_{\alpha}\right)}\left(\left\{\sigma_{n}\right\}\right)}$, $\Omega_{V_{\beta}\left(K_{\alpha}\right)}\left(\left\{\sigma_{n}\right\}\right)=-\sum_{s, r} 2 l_{1 s} \sigma_{s} \tau_{s r}^{(r)} n_{r}\left(V_{\alpha}\right)+\sum_{r}\left(2 l_{2 r}-1\right) n_{r}\left(V_{\alpha}\right)$ where $n_{r}$ is equal to the number of times that $T_{r}$ appears in $V_{\alpha}$ minus the number $T_{r}^{-1}$ appears and

$$
\tilde{Z}_{0}\left(K_{n} ; l_{1 n}, l_{2 n}\right)=\frac{(-1)^{2 l_{1 n}+2 l_{2 n}-1}}{4^{2 l_{1 n}} K_{n}^{\frac{3}{2}}\left[1+(-1)^{2 l_{2 n}} \sqrt{K_{n}} K_{n}^{l_{1 n}}\right]^{2-2 l_{1 n}}}
$$

have also been calculated. ${ }^{36}$ 
An alternative expression for the holomorphic part of the measure is

$$
\begin{aligned}
Z_{L}^{(g)}\left(\left\{K_{n}, Z_{1 n}, Z_{2 n}\right\}\right)= & \tilde{Z}_{0}^{(g)}\left(\left\{K_{n}, \xi_{1 n}, \xi_{2 n}\right\}\right) \Upsilon_{m}^{(g)}\left(\left\{K_{n}, Z_{1 n}, Z_{2 n}\right\}, L\right) \\
& \cdot H\left(\left\{K_{n}, Z_{1 n}, Z_{2 n}\right\}\right) \\
\cdot & \prod_{n=1}^{g}\left[\frac{(-1)^{2 l_{1 n}+2 l_{2 n}-1} 16^{2 l_{1 n}}}{K_{n}^{\frac{\left(3-2 l_{1 n}\right)}{2}}} \prod_{p=1}^{\infty} \frac{\left(1+(-1)^{2 l_{2 n}} K_{n}^{p} K_{n}^{\frac{\left(2 l_{1 n}-1\right)}{2}}\right)^{8}}{\left(1-K_{n}^{p}\right)^{8}}\right]
\end{aligned}
$$

where $\tilde{Z}_{0}^{(g)}\left(\left\{K_{n}, \xi_{1 n}, \xi_{2 n}\right\}\right)$ is evaluated at zero odd Schottky parameter. ${ }^{37}$ From this formulation, and noting the designation of spin structures in equation (4.22), it is clear that when $l_{1 n}=\frac{1}{2}$ and the spin structure for the $n^{\text {th }}$ handle is $\left(+\sigma_{h_{n}}\right), Z_{L}^{(g)} \rightarrow K_{n}^{-1}$ as $K_{n} \rightarrow 0$, while if $l_{1 n}=0$, so that an even spin structure $\left(-\sigma_{h_{n}}\right)$ marks the $n^{t h}$ handle, $Z_{L}^{(g)} \rightarrow K_{n}^{-\frac{3}{2}}$ as $K_{n} \rightarrow 0$, consistent with the result for the degeneration limit of the measure for the Neveu-Schwarz sector.

Given the latter choice of $\left\{l_{1 n}\right\}$, the sum over the $2^{g}$ spin structures associated with the different assignments of either 0 or $\frac{1}{2}$ for $\left\{l_{2 n}\right\}$ softens the divergence of the holomorphic part of the integrand to $K_{n}^{-1}$, and the combinination of this term with the $\left[\ln \left|K_{n}\right|\right]^{-5}$ factor that arises from the super-period matrix produces a finite integral in the degeneration limit $\left|K_{n}\right| \rightarrow 0$.

It is known that the strength of the divergence in a degeneration limit is determined by the conformal weight of state propagating along the tube. With the moduli space near the compactification divisor $\mathcal{D}$ parametrized by $\left(m_{\mathcal{D}}, q\right)=\left(m, x_{1}, x_{2}, q\right)$, where $x_{1}$ and $x_{2}$ are the punctures on the surface $m$ resulting from the removal of a node and $q$ is a complex variable defining the opening of the node, and with $\rho\left(m_{\mathcal{D}}\right)$ denoting the density matrix of states on the two boundary circles at the end of the tube, ${ }^{38}$, the factorization of the partition function is given by

$$
\begin{aligned}
Z & =\sum_{\Phi} q^{h_{\Phi}} \bar{q}^{\bar{h}_{\Phi}}\left\langle\Phi\left|\rho\left(m_{\mathcal{D}}\right)\right| \Phi\right\rangle \\
& =Z(m, \bar{m}) \sum_{\Phi} q^{h_{\Phi}} \bar{q}^{\bar{h}_{\Phi}}\left\langle\Phi\left(x_{1}\right) \Phi\left(x_{2}\right)\right\rangle_{m}
\end{aligned}
$$

where $h_{\Phi}$ is the conformal weight of the field $\Phi$. An additional contribution arises from the ghost fields so that the measure contains factors of the type $q^{h_{\Phi}-\epsilon_{g h}} \bar{q}^{\bar{h}_{\Phi}-\bar{\epsilon}_{g h}}$.

In the Neveu-Schwarz sector of the superstring, $\epsilon_{g h}=\frac{1}{2}$ and the conformal weight of the tachyon vertex operator is $h_{\Phi}=\frac{1}{2} k^{2}=-\frac{1}{2},{ }^{39}$ as the squared mass of the Neveu-Schwarz tachyon is half of the squared mass of the tachyon in the bosonic string spectrum, so that the amplitude is proportional to

$$
\int \frac{d^{2} K}{K \bar{K}} \frac{1}{\left(\log |K|^{-1}\right)^{5}}
$$

where the factor $\left(\log |K|^{-1}\right)^{-\frac{D}{2}}$ has been included upon the pinching of the A-cycles. 
The dilaton vertex operator belongs to the set of massless vertex operators for the fermionic string 40

$$
V_{\text {massless }}(k, \epsilon)=\int d^{2} z d^{2} \theta \epsilon D X^{\mu} \bar{D} X^{\nu} e^{i k \cdot X} \quad k^{\mu} \epsilon_{\mu \nu}=k^{\nu} \epsilon_{\mu \nu}=0
$$

with conformal weight $h_{\Phi}>-\frac{1}{2}$, so that the resulting integral

$$
\int d^{2} q q^{h_{\Phi}-\frac{1}{2}} \bar{q}^{\bar{h}_{\Phi}-\frac{1}{2}}
$$

is finite when $h_{\Phi}>-\frac{1}{2}$ and, when $q$ is the multiplier $\mathrm{K}$, for $h_{\Phi}=-\frac{1}{2}$.

In the Ramond sector, the energy $\epsilon_{g h}=0,{ }^{40}$ so that the finiteness of the integral is improved in the degeneration limits of both $A-$ and $B$-cycles.

Finiteness of superstring amplitudes at arbitrary genus has been demonstrated in the lightcone gauge ${ }^{7}$, and using the twistor-string formalism, where the amplitude contains picturechanging operators, $F^{ \pm}=\left[Q, \xi^{ \pm}\right]$, with $Q$ being a BRST operator derived from the energymomentum tensor and ghosts and $\xi^{ \pm}$are spin- 0 fermions required in the expression for the bosonized ghosts. ${ }^{7}$ Constructing an $N$-punctured, genus- $g$ Riemann surface by sewing threepunctured spheres, labelling the radii $R_{j}, j=1, \ldots, 3 g-3+N$ of the sewed punctures as $K_{n}$, $H_{m}, B_{m}$ and $L_{i}$ (for external legs), and considering the limits $\left\{\left|R_{j}\right| \rightarrow 0\right\}$, the locations of the picture-changing operators may be chosen to be arbitrary points on the $2 g-2+N$ spheres. ${ }^{7}$

Since a change in the location of $F^{ \pm}$implies that $[Q, \xi] \rightarrow[Q, \xi]+\oint d z\left[Q, \partial_{z} \xi\right]$, the contour of $Q$ initially surrounds the curve joining the initial and final positions of the picture-changing operators. ${ }^{41}$ In the degeneration limit $H_{i} \rightarrow 0$, where the Riemann surface splits into two components of genus $g-2$ and 1 , the contour of $Q$ can be pulled off the picture-changing operator to surround the three punctures of $S_{n+g+N}$.

Around each of the sewed punctures is a closed loop $C_{i}$ of radius $R_{i}$ and the contribution from each of the Beltrami differentials is

$$
\left|\prod_{i=1}^{3 g-3+N} \oint_{C_{i}} \frac{y_{i} b\left(y_{i}\right)}{R_{i}} d y_{i}\right|^{2}
$$

Around the first puncture is the contour integral involving the Beltrami differential for $K_{n}$, while the operator at the third puncture is represented by the factor

$$
\left|c \exp \left(-\phi^{+}-\phi^{-}\right) \exp \left(h^{+}+h^{-}\right) \psi^{+} \psi^{-}\right|^{2}
$$

where $\mathrm{c}$ is a right-moving boson of spin- $\frac{1}{2}, \phi^{ \pm}$are two scalar bosons of screening charge 2 defining the bosonized ghosts, $h^{ \pm}$are a pair of right-moving spin- 0 fermions. ${ }^{7}$ Anticommuting Q with the Beltrami differential for $K_{n}$ produces a derivative with respect to $K_{n}$. This total derivative does not represent a divergence because the amplitude already has been shown to be 
finite at the boundary $K_{n}=0$. Anticommutation of $\mathrm{Q}$ with the operator at the third puncture produces no terms with zero-modes of the spin- 0 fermions $\psi^{ \pm}$, and therefore the amplitude is independent of the locations of the picture-changing operators. Since all of the picturechanging operators may be moved to the third puncture, the zero-modes of $\psi^{+}$and $\psi^{-}$cancel, implying that this amplitude associated with the genus 1 component of the Riemann surface vanishes. ${ }^{7}$ This leaves the contribution from the boundary of the $K_{n}$ region and, in this sense, it represents a transfer from an integral involving $H_{n-2}$ to a finite integral involving $K_{n}$. Consequently, divergences are absent in the (Type IIB Green-Schwarz) superstring amplitudes in the degeneration limit $H_{m} \rightarrow 0$. A similar argument may be used to demonstrate that the amplitudes are also finite in the $B_{m} \rightarrow 0$ limit, which defines the degeneration of the $C_{m}$-cycle. Since the $C_{m}$-cycles are linear combinations of the $A_{n}$-cycle and $B_{n}$-cycles, the divergence in the $\left|B_{m}\right| \rightarrow 0$ limit disappear once the superstring amplitudes are demonstrated to be finite in the limits $\left|K_{n}\right| \rightarrow 0$ and $\left|\xi_{1 n}-\xi_{2 n}\right| \rightarrow 0$.

An estimate of the amplitudes based on the measure for the Neveu-Schwarz sector and the Ramond sector can be obtained by applying modular transformations to the spin structures in these two sectors. In terms of the sums over spin structures,

$$
\begin{aligned}
\int_{F_{g}} \sum_{(a) \in S_{r}^{\prime}} \eta_{(a)} I_{N, g}^{(a)} & =\int_{F_{g}} \sum_{\left(a_{e}\right)=1}^{2^{g-1}} \eta_{\rho_{r}\left(a_{e}\right)} I_{N, g}^{\rho_{r}\left(a_{e}\right)}+\int_{F_{g}} \sum_{\left(a_{o}\right)=1}^{2^{g-1}} \eta_{\rho_{r}\left(a_{0}\right)} I_{N, g}^{\rho_{r}\left(a_{o}\right)} \\
& =\int_{\rho_{r}^{-1}\left(F_{g}\right)} \sum_{\left(a_{e}\right)=1}^{2^{g-1}} \eta_{\left(a_{e}\right)} I_{N, g}^{\left(a_{e}\right)}+\int_{\rho_{r}^{-1}\left(F_{g}\right)} \sum_{\left(a_{0}\right)=1}^{2^{g-1}} \eta_{\left(a_{0}\right)} I_{N, g}^{\left(a_{0}\right)}
\end{aligned}
$$

The integral involving the spin structures $\left\{\rho_{r}\left(a_{e}\right)\right\} \cup\left\{\rho_{r}\left(a_{0}\right)\right\}$ has been converted to an integral involving the spin structures $\left\{\left(a_{e}\right)\right\} \cup\left\{\left(a_{0}\right)\right\}=S_{2}^{\prime}$, with a different integration region in the Schottky parameter space.

The effect of a modular transformation $\rho_{r}^{-1}$ on the fundamental region $F_{g}$ is that of a mapping to another domain in the Siegel upper half plane. Since the condition $|\operatorname{det}(C \tau+D)| \geq$ 1 is not necessarily satisfied by the transformed period matrix, the upper limit for $\left|K_{n}\right|$ is not constrained by the inequality

$$
\epsilon_{0}^{\prime} \leq \exp \left(-b^{\frac{1}{g}}+\frac{1}{g} \sum_{n=1}^{g} l_{n n}\right)
$$

where $b$ is a lower bound for $\operatorname{det}(\operatorname{Im} \tau)$ and $s_{n n}$ is the supremum of the lower bounds for $\sum_{\alpha}^{(n, n)} \ln \left|\frac{\xi_{1 n}-V_{\alpha} \xi_{2 n}}{\xi_{1 n}-V_{\alpha} \xi_{2 n}} \frac{\xi_{2 n}-V_{\alpha} \xi_{1 n}}{\xi_{2 n}-V_{\alpha} \xi_{2 n}}\right|$. While the domain $\rho_{r}^{-1}\left(F_{g}\right)$ is defined by different limits for the Schottky group parameters, the shift of the integration from $F_{g}$ to $\rho_{r}^{-1}\left(F_{g}\right)$ will change the bound on the absolute values of the integrals by an exponential factor $\chi(g)^{g}$.

$$
\sum_{r=1}^{2^{g+1}-2} \int_{F_{g}} \sum_{(a) \in S_{r}^{\prime}} \eta_{(a)} I_{N, g}^{(a)}=\int_{F_{g}} \sum_{(i)=1}^{2^{2 g}} \eta_{(i)} I_{N, g}^{(i)}+\sum_{s} \int_{F_{g}} \sum_{(b) \in S_{s}^{\prime \prime}} \eta_{(b)} I_{N, g}^{(b)}
$$


where the set $\left\{S_{s}^{\prime \prime}\right\}$ consists of $2^{g-1}$ spin structures.

\section{Bounds for the Generic N-Point Superstring Scattering Amplitudes}

The vanishing of $\mathrm{N}$-point amplitudes for $N<4$ provides the first indication that the superstring amplitudes do not necessarily grow at a factorial rate with respect to the genus. Similarly, vanishing of superstring amplitudes has been established when $g+N \leq 8 .{ }^{42}$ The only result that has been established thus far the superstring scattering amplitudes at high genus is a consequence of a technical argument relating $\mathrm{N}=2$ string theories with topological field theories. This connection implies that there is a relation between special type II string amplitudes in orbifold and Calabi-Yau backgrounds and topological string amplitudes at any given genus. ${ }^{43,44}$ It is therefore of interest to be able to estimate the generic superstring scattering amplitude with an arbitrary number of vertex operators, receiving contributions from all genus. This follows from the bounds in this paper because the $\mathrm{N}$-point $g$-loop scattering amplitude is typically given by

$$
\begin{gathered}
A_{N, g}=\int_{s M_{g}} d \mu_{g} \int_{s \Sigma_{g}} d^{2} z_{1} \ldots d^{2} z_{N} d \theta_{1} . . d \theta_{N} d \bar{\theta}_{1} \ldots d \bar{\theta}_{N} \sqrt{g\left(z_{1}, \bar{z}_{1}\right)} \ldots \sqrt{g\left(z_{N}, \bar{z}_{N}\right)} \\
\left\langle\mathcal{V}_{1}\left(z_{1}, \theta_{1}, \bar{z}_{1}, \bar{\theta}_{1}\right) \ldots \mathcal{V}_{N}\left(z_{N}, \theta_{N}, \bar{z}_{N}, \bar{\theta}_{N}\right)\right\rangle
\end{gathered}
$$

which may be bounded by

$$
\begin{gathered}
c_{N}\left|\int_{s M_{g}-\mathcal{N}\left(s D_{g}\right)} d \mu_{g}\right| \mid \int_{\Sigma_{g}} d z_{1} d \bar{z}_{1} \ldots d z_{N} d \bar{z}_{N} \sqrt{g\left(z_{1}, \bar{z}_{1}\right)} \ldots \sqrt{g\left(z_{N}, \bar{z}_{N}\right)} \\
\left\langle V_{1}\left(z_{1}, \bar{z}_{1}\right) \ldots V_{N}\left(z_{N}, \bar{z}_{N}\right)\right\rangle \mid \\
+c_{N}\left|\int_{\mathcal{N}\left(s D_{g}\right)} d \mu_{g} \int_{\Sigma_{g}} d^{2} z_{1} \ldots d^{2} z_{N} \sqrt{g\left(z_{1}, \bar{z}_{1}\right)} \ldots \sqrt{g\left(z_{N}, \bar{z}_{N}\right)}\left\langle V_{1}\left(z_{1}, \bar{z}_{1}\right) \ldots V_{N}\left(z_{N}, \bar{z}_{N}\right)\right\rangle\right|
\end{gathered}
$$

where $\mathcal{N}\left(s D_{g}\right)$ is a neighbourhood of the compactification divisor in supermoduli space and $c_{N}$ is a coefficient which increases exponentially as a function of $N$. For the $R=-1$ slice of Teichmuller space,

$$
\frac{1}{2 \pi} \int_{\Sigma_{g}} d^{2} \xi \sqrt{g} R=2-2 g
$$

and since a subtraction procedure can be used to remove divergences ${ }^{45}$ in the correlation function associated with the coincidence of vertex operators to bound its magnitude $\left|\left\langle V_{1}\left(z_{1}, \bar{z}_{1}\right) \ldots V_{N}\left(z_{N}, \bar{z}_{N}\right)\right\rangle\right| \leq V_{N}^{\max }$, it follows that the upper bound (5.2) is less than

$$
(4 \pi(g-1))^{N} \cdot c_{N} \cdot V_{N}^{\max } \cdot \frac{g !(g-2) !(g-1) !}{(3 g-3) !}\left(B_{K}+B_{K}^{\prime}+B_{H}+B_{H}^{\prime}+B_{B}+B_{B}^{\prime}\right)^{3 g-3}
$$

Lower bounds of this type may also be derived provided there is a suitable lower bound that can be used for expressions containing the supermoduli space integral. Since this particular 
integral, after the sum over spin structures, actually vanishes, the lower bound that should not be determined strictly by the magnitude of this integral; rather, it should allow for the weighting of the spin structures to be altered in the integration of the correlation function over supermoduli space, leading to a non-vanishing amplitude.

The genus-dependence of the special scattering amplitudes can be obtained from the general estimate (5.4). Specifically, the genus-g type II string amplitude $A_{2 g, g}$ in an orbifold or CalabiYau background, with $2 \mathrm{~g}$ vertex operators including $2 \mathrm{~g}-2$ graviphotons and 2 gravitons, is equal to

$$
A_{2 g, g}=(g !)^{2} F_{g}
$$

where $F_{g}$ is the partition function of a topological string theory. The partition function for a topological field theory defined over a particular manifold $\mathrm{M}$ typically is given by the product of the partition function over the submanifolds $M_{i}$, such that $\mathrm{M}$ is the topological sum $\cup_{i} M_{i}$, so that the partition function for a genus- $g$ surface $Z_{g}$ would be $c^{g} Z_{1}^{g}$. For a topological gravity theory, the genus- $g$ partition function will also involve a contribution from a supermoduli space integral associated with modular deformations along the collars joining the tori. Since the magnitude of this supermoduli integral has already been estimated, the genus-dependence of the partition function $F_{g}$ should still be exponential, and indeed, this has been established in several papers. ${ }^{42,46,47}$ Consequently, the type II string amplitude is $A_{2 g, g}=c_{1} c_{2}^{g}(g !)^{2}$, consistent with the bound (5.4) upon setting $\mathrm{N}$ equal to $2 \mathrm{~g}$.

These results provide further confirmation of the higher degree of finiteness in superstring theory in the large-genus limit. The exponential function (5.4) implies a similar bound for the for higher-point superstring amplitudes, and the perturbation series can be made to converge for an appropriate choice of the string coupling constant or dilaton expectation value.

\section{Estimates of Moduli Space Integrals for the Category of Effectively Closed Infinite-Genus Surfaces and the String Coupling Constant}

Study of the large-genus limit in bosonic string theory shows that the requirement of a finite-size interaction region restricts the surfaces to be either closed or effectively closed infinite-genus surfaces. ${ }^{21}$ Both types of surfaces always can be constructed by placing handles on spheres, which decrease in size to zero when there are an infinite number, thereby breaking the bound on the minimum length of closed geodesics. The genus-independent cut-off on this length excludes spheres with a sufficiently large number of handles, and therefore, it represents a large-genus cut-off as well.

The absence of divergences in N-point superstring amplitudes at any given finite order in perturbation theory implies that the restriction on the minimum length of closed geodesics should be removed. Effectively closed infinite-genus surfaces, besides completing the domain of string perturbation theory, may have an essential role in the superstring path integral. Configurations of isometric circles arising in the super-Schottky uniformization of these surfaces shall then be included in the supermoduli space integral. The ranges $\frac{\epsilon_{0}}{n^{q^{\prime \prime}}} \leq\left|K_{n}\right| \leq \frac{\epsilon_{0}^{\prime}}{n^{q^{\prime \prime}}}, n=$ 
$1, \ldots, g, \quad q^{\prime \prime}>1$, and $\frac{\delta_{0}}{n^{q^{\prime \prime \prime}}} \leq\left|\xi_{1 n}-\xi_{2 n}\right| \leq \frac{\delta_{0}^{\prime}}{n^{q^{\prime \prime \prime}}}, n=1, \ldots, g, \quad q^{\prime \prime \prime}>\frac{1}{2}$, therefore will be

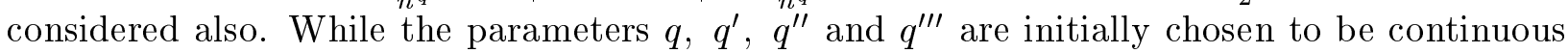
parameters, to avoid overcounting in the path integral, it is necessary to select a discrete set of values. Specifically, it is sufficient to sum over the values

$$
\begin{array}{ll}
q \leq q_{N}^{\prime}=N \frac{\ln \left(\frac{\epsilon_{0}^{\prime}}{\epsilon_{0}}\right)}{2 \ln g} \quad N=0,1, \ldots,\left[\frac{\ln g}{\ln \left(\frac{\epsilon_{0}^{\prime}}{\epsilon_{0}}\right)}\right] \\
q_{\tilde{N}}^{\prime \prime}=1+\tilde{N} \frac{\ln \left(\frac{\epsilon_{0}^{\prime}}{\epsilon_{0}}\right)}{\ln n} & \tilde{N}=1,2, \ldots \\
q_{\tilde{N}^{\prime}}^{\prime \prime \prime}=\tilde{N}^{\prime} \frac{\ln \left(\frac{\delta_{0}^{\prime}}{\delta_{0}}\right)}{\ln n} & \tilde{N}^{\prime}=0,1,2, \ldots
\end{array}
$$

Non-overlapping of the ranges $\frac{\epsilon_{0}}{n^{q^{\prime \prime}}} \leq\left|K_{n}\right| \leq \frac{\epsilon_{0}^{\prime}}{n^{q^{\prime \prime}}}$ requires that the set $\left\{q_{\tilde{N}}^{\prime \prime}\right\}$ be selected so that

$$
\begin{aligned}
\frac{\epsilon_{0}^{\prime}}{n^{q_{\tilde{N}+1}^{\prime \prime}}} & =\frac{\epsilon_{0}}{n^{q_{\tilde{N}}^{\prime \prime}}} \\
q_{\tilde{N}+1}^{\prime \prime}-q_{\tilde{N}}^{\prime \prime} & =\frac{\ln \left(\frac{\epsilon_{0}^{\prime}}{\epsilon_{0}}\right)}{\ln n}
\end{aligned}
$$

and the last equality is satisfied by the sequence $\left\{q_{\tilde{N}}^{\prime \prime}\right\}$ in equation (6.1). Similarly, the values of $q_{\tilde{N}^{\prime}}^{\prime \prime \prime}$, are consistent with non-overlap of the intervals for $\left|\xi_{1 n}-\xi_{2 n}\right|$, given the limits for $\left|K_{n}\right|$.

The super-fixed-point integral contains a factor $\left|\xi_{1 n}-\xi_{2 n}\right|^{-2}$, so that it will grow as $n^{2 q^{\prime \prime \prime}}$, which would give rise to a divergence when $q^{\prime \prime \prime}>\frac{1}{2}$. However, it is also essential to eliminate the overcounting of surfaces, which would arise upon including every value of $q_{\tilde{N}^{\prime}}^{\prime \prime \prime}, \tilde{N}^{\prime}$ integer. In particular, surfaces corresponding to the limits $\frac{\delta_{0}}{n^{2 q^{\prime \prime \prime}}}<\left|\xi_{1 n}-\xi_{2 n}\right|<\frac{\delta_{0}^{\prime}}{n^{2 q^{\prime \prime \prime}}}$ can be obtained from surfaces corresponding to $\frac{\delta_{0}}{n^{q^{\prime \prime \prime}}}<\left|\xi_{1 n}-\xi_{2 n}\right|<\frac{\delta_{0}^{\prime}}{n^{q^{\prime \prime \prime}}}$ by pinching all handles other than those with the index $n^{2}$ in the sequential labelling, removing the nodes and deforming the surface by flattening the remaining portion of the handle on the sphere. Pinching one handle produces a surface at the boundary of moduli space, or equivalently, at the boundary of a string vertex, denoted by $\partial \mathcal{M}_{g, 0}$ and $\partial \mathcal{V}_{g, 0}$ respectively at genus g. ${ }^{48}$ Removing the two new punctures in the manner using an analytic map ${ }^{37}$ transforms the string vertex from $\mathcal{V}_{g-1,2}$ to $\mathcal{V}_{g-1,0}$, which lies in the compactified moduli space $\overline{\mathcal{M}}_{g-1,0}$. At infinite genus, this procedure still produces a surface with an infinite number of handles lying in $\overline{\mathcal{M}}_{\infty}$ [the number of ends of an infinite-genus surface is not required here]. Thus the initial integration over the domain in $\overline{\mathcal{M}}_{\infty}$ associated with the configurations of isometric circles with the range for $\left|\xi_{1 n}-\xi_{2 n}\right|$ given by $\left[\frac{\delta_{0}}{n^{q^{\prime \prime}}}, \frac{\delta_{0}^{\prime}}{n^{q^{\prime \prime \prime}}}\right]$, includes integration over the range $\left[\frac{\delta_{0}}{n^{2 q^{\prime \prime}}}, \frac{\delta_{0}^{\prime}}{n^{2 q^{\prime \prime r}}}\right]$ up to pinching of handles. The process of pinching the handles is described, however, by the degeneration limits $\left|K_{m}\right| \rightarrow 0, m \neq n^{2}, n \in \mathbb{Z}$, which will be shown not to lead to any new divergences in the moduli space integral. Pinching a handle representing a degeneration limit $\left|K_{m}\right| \rightarrow 0, m \neq n^{2}$ 
gives a finite addition to the moduli space integral. Similarly, creating a handle gives rise to a finite addition to the moduli space integral, which becomes progressively smaller as the handles decrease in size. If the sum of these contributions is also finite, then the difference between the integral over the range $\left\{\xi_{1 n}-\xi_{2 n} \mid \sim \frac{1}{n^{2} q^{\prime \prime r}}\right\}$ and the integral over the range $\left|\xi_{1 n}-\xi_{2 n}\right| \sim \frac{1}{n^{q^{\prime \prime \prime}}}$ is finite, which is necessary to consistently define the integral over effective closed infinite-genus surfaces. This result can be verified using handle operators ${ }^{49}$.

Since this argument can be repeated an arbitrary number of times, overcounting will be eliminated by restricting the fixed-point integral to the range $\left\{\left|\xi_{1 n}-\xi_{2 n}\right| \sim \frac{1}{n^{q^{\prime \prime \prime}}}\right\}, x>q^{\prime \prime \prime}>0$, with $\mathrm{x}$ being a small positive number. It is therefore necessary to choose

$$
\frac{\ln (\ln n)}{\ln \left(\frac{\delta_{0}^{\prime}}{\delta_{0}}\right)}<\tilde{N}_{\text {max }}^{\prime} \leq x \frac{\ln n}{\ln \left(\frac{\delta_{0}^{\prime}}{\delta_{0}}\right)}
$$

An upper bound for the integrals over the fixed points would then grow as $n^{2 x}$.

Another class of surfaces that might be included in the superstring path integral are spheres with an infinite number of handles decreasing in size and accumulating to a point. These surfaces may be constructed using infinitely generated groups of Schottky type, joining together isometric circles by projective transformations in the extended complex plane. ${ }^{21}$ Labelling the handles by the index $n$, the decrease in the square of the radii of the isometric circles, $\left|\gamma_{n}\right|^{-2} \sim \frac{1}{n^{q^{\prime \prime}}}$ characterizes the surfaces to be included in the path integral. From the leading behaviour of the multiplier integral, the contribution of a configuration of isometric circles with $q_{\tilde{N}}^{\prime \prime}$ given in equation (6.1) may be estimated.

$$
\begin{aligned}
\int_{\left|K_{n}\right| \sim \frac{1}{n q_{\tilde{N}}^{\prime \prime}}} \frac{d\left|K_{n}\right|}{\left|K_{n}\right|\left(\ln \frac{1}{\left|K_{n}\right|}\right)^{5}} & =\frac{1}{4}\left[q_{\tilde{N}}^{\prime \prime} \ln n-\ln \epsilon_{0}^{\prime}\right]^{-4}-\frac{1}{4}\left[q_{\tilde{N}}^{\prime \prime} \ln n-\ln \epsilon_{0}\right]^{-4} \\
& =\left(q_{\tilde{N}}^{\prime \prime} \ln n\right)^{-5} \ln \left(\frac{\epsilon_{0}^{\prime}}{\epsilon_{0}}\right) \\
& =\left(\frac{\ln n}{\ln \left(\frac{\epsilon_{0}^{\prime}}{\epsilon_{0}}\right)}+\tilde{N}\right)^{-5}\left[\ln \left(\frac{\epsilon_{0}^{\prime}}{\epsilon_{0}}\right)\right]^{-4}
\end{aligned}
$$

While the measure for a Neveu-Schwarz-Ramond sector includes the product over $n$ of the integrals (6.4), it is more useful to first sum over the index $\tilde{N}$ as this will allow for the inclusion of all possible combinations of limits for the multipliers and fixed points.

The constraints defining the fundamental region of the modular group, $(\operatorname{Im} \tau)_{s s} \geq(\operatorname{Im} \tau)_{r r}, s \geq r$ reduce the integration range of the Schottky group variables. However, they are only relevant when the intervals $\left[\frac{\epsilon_{0}}{n_{s}^{q^{\prime \prime}}}, \frac{\epsilon_{0}^{\prime}}{n_{s}^{q^{\prime \prime}}}\right]$ and $\left[\frac{\epsilon_{0}}{n_{r}^{q^{\prime \prime}}}, \frac{\epsilon_{0}^{\prime}}{n_{r}^{q^{\prime \prime}}}\right]$ overlap, implying

$$
\frac{n_{s}}{n_{r}} \leq\left(\frac{\epsilon_{0}^{\prime}}{\epsilon_{0}}\right)^{\frac{1}{q^{\prime \prime}}}
$$


Assuming temporarily that the genus is finite and an integer between $\left(\frac{\epsilon_{0}^{\prime}}{\epsilon_{0}}\right)^{\frac{y-1}{q^{\prime \prime}}}$ and $\left(\frac{\epsilon_{0}^{\prime}}{\epsilon_{0}}\right)^{\frac{y}{q^{\prime \prime}}}$, for some integer $y$, the restrictions on each group of multipliers $K_{n}$ with $n$ lying between the numbers $1,\left(\frac{\epsilon_{0}^{\prime}}{\epsilon_{0}}\right)^{\frac{1}{q^{\prime \prime}}},\left(\frac{\epsilon_{0}^{\prime}}{\epsilon_{0}}\right)^{\frac{2}{q^{\prime \prime}}}, \ldots$ leads to a combinatorial factor

$$
\begin{aligned}
& \frac{1}{\left(\left(\frac{\epsilon_{0}^{\prime}}{\epsilon_{0}}\right)^{\frac{1}{q^{\prime \prime}}}-1\right) !\left(\left(\frac{\epsilon_{0}^{\prime}}{\epsilon_{0}}\right)^{\frac{2}{q^{\prime \prime}}}-\left(\frac{\epsilon_{0}^{\prime}}{\epsilon_{0}}\right)^{\frac{1}{q^{\prime \prime}}}\right) ! \ldots\left(g-\left(\frac{\epsilon_{0}^{\prime}}{\epsilon_{0}}\right)^{\frac{y-1}{q^{\prime \prime}}}\right) !} \\
& =\frac{g !\left(\left(\frac{\epsilon_{0}^{\prime}}{\epsilon_{0}}\right)^{\frac{y}{q^{\prime \prime}}}-\left(\frac{\epsilon_{0}^{\prime}}{\epsilon_{0}}\right)^{\frac{y-1}{q^{\prime \prime}}}\right) !}{\left(\frac{\epsilon_{0}^{\prime}}{\epsilon_{0}}\right)^{\frac{y}{q^{\prime \prime}}} !\left(g-\left(\frac{\epsilon_{0}^{\prime}}{\epsilon_{0}}\right)^{\frac{y-1}{q^{\prime \prime}}}\right) !}\left(\frac{\left(\frac{\epsilon_{0}^{\prime}}{\epsilon_{0}}\right)^{\frac{1}{q^{\prime \prime}}}}{\left.\left(\left(\frac{\epsilon_{0}^{\prime}}{\epsilon_{0}}\right)^{\frac{1}{q^{\prime \prime}}}-1\right)^{1-\left(\frac{\epsilon_{0}}{\epsilon_{0}^{\prime}}\right)^{\frac{1}{q^{\prime \prime}}}}\right)^{\frac{\left(\frac{\epsilon_{0}^{\prime}}{\epsilon_{0}}\right)^{\frac{y+1}{\epsilon_{0}^{\prime}}}-1}{\epsilon^{\frac{1}{q^{\prime \prime}}}}-1}} \frac{1}{g !}\right. \\
& <\frac{1}{g !} 2^{\frac{\left(\frac{\epsilon_{0}^{\prime}}{\epsilon_{0}}\right)^{\frac{2}{q^{\prime \prime}}} g-1}{\left(\frac{\epsilon_{0}^{\prime}}{\epsilon_{0}}\right)^{\frac{1}{q^{\prime \prime}}} g-1}}
\end{aligned}
$$

Defining the variable

$$
t_{\tilde{N}}=\left(\frac{\epsilon_{0}^{\prime}}{\epsilon_{0}}\right)^{\frac{1}{q_{\tilde{N}}^{\prime \prime}}} \simeq\left(\frac{\epsilon_{0}^{\prime}}{\epsilon_{0}}\right)^{\frac{1}{\tilde{N}} \frac{\ln n}{\ln \frac{\epsilon_{0}^{\prime}}{\epsilon_{0}}}=n^{\frac{1}{\tilde{N}}}}
$$

one finds that the combinatorial factor $(6.6)$ is less than $\frac{1}{(g !)^{t} \tilde{N}^{-1}}$.

The sum of the fixed-point integrals over all values of $\tilde{N}^{\prime}$ grows as

$$
\left(\frac{1}{\delta_{0}^{2}}-\frac{1}{\delta_{0}^{\prime 2}}\right) \sum_{\tilde{N}^{\prime}=0}^{\tilde{N}_{\max }^{\prime}} n^{2 q_{\tilde{N}^{\prime}}^{\prime \prime \prime}} \simeq \frac{1}{\delta_{0}^{2}} n^{2 x}
$$

Multiplying this sum by the estimate (6.6) and dividing by the combinatorial factor arising from the action of the modular group gives

$$
\begin{aligned}
\frac{1}{(g !)^{t} \tilde{N}_{\text {max }}^{\prime}-1} & \left(\ln \frac{\epsilon_{0}^{\prime}}{\epsilon_{0}}\right)^{-4 g} \frac{1}{\delta_{0}^{2 g}} \prod_{n=1}^{g} n^{2 x} \zeta\left(5, \frac{\ln n}{\ln \frac{\epsilon_{0}^{\prime}}{\epsilon_{0}}}-1\right) \\
& \rightarrow \frac{1}{\left(4 \delta_{0}^{2}\right)^{g}} \prod_{n=1}^{g} \frac{1}{(\ln n)^{4}}
\end{aligned}
$$

in the limit $g \rightarrow \infty$ when $n^{\frac{1}{\hat{N}_{\text {max }}^{\prime}}}-1=2 x$. Since it is assumed that $x<1$, the upper limit in equation (6.3) implies that this bound will be adequate if $x \leq \frac{1}{\sqrt{2}}\left(\ln \left(\frac{\delta_{0}^{\prime}}{\delta_{0}}\right)\right)^{\frac{1}{2}}$. As the product 
in equation (6.9) tends to zero as $g \rightarrow \infty$, the integrals corresponding to the range $\left|\xi_{1 n}-\xi_{2 n}\right| \sim$ $\frac{1}{n^{2 q^{\prime \prime}}}, q_{\tilde{N}^{\prime}}^{\prime \prime \prime}>\frac{1}{\sqrt{2}}\left(\ln \left(\frac{\delta_{0}^{\prime}}{\delta_{0}}\right)\right)^{\frac{1}{2}}$ would be the limit of an exponential function of the genus for each value of $\tilde{N}^{\prime}$. Thus, the magnitude of that part of the moduli space integral including spheres having $g$ handles, with a decreasing cross-sectional area in the intrinsic metric, near a single accumulation point, is therefore a countable sum of contributions depending exponentially on the genus as $g \rightarrow \infty$.

Based on the limits chosen for $\left|K_{n}\right|$ and $\left|\xi_{1 n}-\xi_{2 n}\right|$, and the interchangeability of the cycles on the Riemann surface under the action of the modular group, it follows that the inclusion of effectively closed surfaces in the path integral requires a lower bound for $\left|\xi_{2 m}\right|$ of the form $\frac{\delta_{2}}{g^{2 \tilde{q}}}$. Given that the limit $\epsilon_{m}$ for $\left|\xi_{2 m}\right|$ then would be defined by the relation $\epsilon_{m}^{m-1}=\frac{\delta_{2}}{g^{2 \tilde{q}}}$ the upper bound (3.11) becomes

$$
\begin{aligned}
(2 \pi)^{g-1} \prod_{m=2}^{g-1} \frac{1}{\epsilon_{m}} & =(2 \pi)^{g-1} \prod_{m=2}^{g-1}\left(\frac{\delta_{2}}{g^{2 \hat{q}}}\right)^{-\frac{1}{m-1}}=(2 \pi)^{g-1}\left(\frac{\delta_{2}}{g^{2 \hat{q}}}\right)^{-\sum_{m=2}^{g} \frac{1}{m-1}} \\
& =(2 \pi)^{g-1}\left(\frac{g^{2 \hat{q}}}{\delta_{2}}\right)^{\psi(g)+\mathcal{C}}
\end{aligned}
$$

where $\mathcal{C}$ is Euler's constant, and thus the contribution of $(2 \pi)^{g-1} e^{2 \hat{q}(l n g)^{2}}$ is subdominant in comparison with an exponential function of the genus.

A sphere with an infinite number of handles may have more than one accumulation point, and generically, an infinite-genus Riemann surface will have a Cantor set of ends, and, the effect of including these surfaces in the superstring path integral now shall be described .

Finiteness of superstring amplitudes at any given order of perturbation theory implies that a cut-off does not need to be introduced in moduli space and and that ambiguities associated with surface terms arising from total derivatives resulting from changes in the locations of picturechanging operators are eliminated. ${ }^{6,50,51}$ In particular, vanishing of the vacuum amplitude at each order in perturbation theory follows from its representation as the integral of a total derivative on the compactified moduli space $\overline{\mathcal{M}}_{g}$. Similarly, the full vacuum amplitude, given by a sum of multi-loop amplitudes, may be regarded as the integral of a total derivative on the universal moduli space $\overline{\mathcal{R}}=\prod_{g=0}^{\infty}\left(\cup_{k=0}^{\infty} S y m^{k}\left(\overline{\mathcal{M}}_{g}\right)\right), \quad{ }^{52}$ and thus, it will be determined by its behaviour at the boundary of this space, and particularly in the infinite-genus limit. Unitarity of the S-matrix in field theory, $T \exp \left[i \int d^{4} x \mathcal{L}_{i n t}(x)\right]$, requires that the vacuum amplitude $\left\langle 0_{\text {in }} \mid 0_{\text {out }}\right\rangle=\left\langle 0_{\text {in }}|S| 0_{\text {in }}\right\rangle$ be non-vanishing, even though the probability can be less than one, since it would be impossible otherwise to build an 'out' Fock space from an 'in' Fock space. It is therefore of interest to find the non-zero contributions to any vacuum amplitude, particularly from sums over surfaces with Dirichlet boundaries and infinite-genus surfaces.

The domain of string perturbation theory can be restricted to closed surfaces and effective closed $O_{G}$ surfaces, with the the infinite-genus surfaces being distinguished by the set of accumulation points of the handles. Based on the cardinality of a Cantor-like set $\mathrm{E}$ or any of 
its subsets, the connection with $O_{G}$ surfaces may be deduced, since the ideal boundary must have zero linear measure and Hausdorff dimension less than one.

The contribution of infinite-genus surfaces to the N-point scattering amplitude will then be

$$
\sum_{n=1}^{\operatorname{card}} \sum_{i=1}^{n_{e}} l i m_{g \rightarrow \infty} \kappa^{2 g-2} \int_{s \mathcal{M}_{\infty,(i)}} \sum_{L, L^{\prime}} d \mu_{\infty,(i),\left(L, L^{\prime}\right)} \prod_{r=1}^{N} d t_{r} d \bar{t}_{r} V_{r}\left(t_{r}, \bar{t}_{r}\right)_{L, L^{\prime}}
$$

where $\left(L, L^{\prime}\right)$ denotes the spin structure and $\left(t_{r}, \bar{t}_{r}\right)$ represents the local coordinate $\left(z_{r}, \theta_{r}, \bar{z}_{r}, \bar{\theta}_{r}\right)$ of the vertex operator on the super-Riemann surface.

It is conceivable that the structure of $\overline{\mathcal{M}}_{\infty}$ is sufficiently different from $\overline{\mathcal{M}}_{g}, g$ finite, that the finiteness condition may select a specific value of the string coupling constant, which could then be compared with phenomenological values arising in unification models. ${ }^{53}$ For a small value of the coupling constant, the amplitude decreases exponentially to zero. It might receive a non-zero contribution at infinite genus because card $\mathrm{E}$ can be regarded as the limit of an exponential function of the genus. The dependence on the genus takes the form $c(\kappa)^{g}$ at finite $g$ and $($ card $E) \lim _{g \rightarrow \infty} c(\kappa)^{g}$ at $g=\infty$. While the standard perturbative result for $c(\kappa)<1$ would be 0 at $g=\infty$, this is now modified by the separate labelling of the endpoints and will be finite and non-zero if $\operatorname{card} E=\lim _{g \rightarrow \infty} \frac{1}{c(\kappa)^{g}}$.

\section{Non-Perturbative Effects and Additional Contributions to the Sum over Surfaces}

Whereas exponential non-perturbative effects of order $\exp \left(-\frac{1}{\kappa^{2}}\right)$ typically arise in quantum field theories as a results of non-Borel summability of the perturbation series, ${ }^{54}$ nonperturbative effects in string theory have been intepreted as a seperate contribution to the sum over string histories associated with the insertion of Dirichlet boundaries to the worldsheets with amplitudes of the form

$$
A_{1}=\exp \left(\langle 1\rangle_{D_{2}}+\ldots\right) A_{1}^{c o n n}
$$

where $\langle 1\rangle_{D_{2}}$ is the disk amplitude with no vertex operators having weight $-\frac{1}{\kappa_{s t r .}} .{ }^{5}$ String divergences which could arise when vertex operators approacha the Dirichlet boundaries, are eliminated by a Fischler-Susskind mechanism, in which amplitudes with different numbers of boundaries cancel. ${ }^{55}$

A formula for the S-matrix including the combinatorics of boundaries on the string worldsheets has been given. ${ }^{56}$ The scattering amplitudes defined in this article arise as the logarithm of the first term in the series expansion of the generating functional

$$
\ln \mathcal{Z}=S^{(0)}+\ln \left[\sum_{n} \frac{1}{n !}\left(\int \prod_{i=1}^{n} d^{D} y_{i}^{\mu}\right) e^{S^{(n) \prime}\left(y_{1}, \ldots, y_{n}\right)}\right]
$$


where $S^{(0)}$ is the path integral over connected worldsheets with no boundaries, $S^{(n)}$ is the functional integral over worldsheets with boundaries fixed at $\mathrm{n}$ points $y_{i}$ and $S^{(n) \prime}$ is $S^{(n)}$ without the zero-boundary term.

The existence of string solitons representing a transition from one solution of the string equations to another background geometry, ${ }^{57}$ and a connection with boundary states of a specific type attached to the worldsheet indicate that a small amplitude, of order $e^{-\frac{1}{\kappa_{s t r}} \text {, }}$ 58,59 arising from a non-perturbative instability, would be consistent with finiteness of the sum over string histories. By analogy with supersymmetric ground states of supergravity theories, the positive-energy theorems can begin with the formal relation

$$
E_{g r}=\langle 0|H| 0\rangle=\left\langle 0\left|\sum_{i}\left\{Q_{\alpha}^{i}, Q_{\alpha}^{\dagger i}\right\}\right| 0\right\rangle
$$

where $i$ denotes the number supersymmetries of the background geometry, $\mathrm{H}$ is an appropriately defined string Hamiltonian and $|0\rangle$ is a vacuum state, encoding information, about the metric of target space. The formal identity can be used to prove results about the energy associated with different background geometries, when the appropriate limit of the operator equation is taken. The boundary states that have recently been shown to described string solitons could provide a way of circumventing the positive-energy theorems. ${ }^{60,61}$ It is known, for example, that there is a soliton in Type II superstring theory which defines a local anti-de Sitter geometry. Since spatial infinity is not included in this region, a one-parameter family of vacua can be defined for the scalar and higher-spin components of the string field. With respect to these new vacua, a transition from the original supersymmetric background to a new local target-space geometry might be allowed, without contradicting either the convergence of the perturbative expansion for the S-matrix or the positive-energy theorem for space-times admitting Killing spinors which are solutions to the classical field equations of a supersymmetric theory.

\section{Consistency with Low-Energy Field Theories, Duality and the Transformation to the Weak-Coupling Regime}

The exponential bounds for the amplitudes, based only on the sum over closed surfaces, are therefore not inconsistent, in this scheme, with the non-perturbative effects that have been expected to be present in string theory. Further support for the estimates of superstring amplitudes in this article can be gleaned from the convergent perturbation expansion for QED with fermions and finite ultraviolet and infrared cut-offs. 4,62 From the work on bosonic string theory, it follows that the ultraviolet cut-off in this theory is equivalent to the Gross-Periwal cut-off, while the infrared cut-off removes the divergences in the infrared limit, analogous to the effect of supersymmetry on string amplitudes. This version of the QED model also may arise in the low-energy limit of superstring theory, providing an explanation for the dependence of the amplitudes on the loop order.

It has been shown that the strong-coupling regime in supersymmetric gauge theories defined by electric variables is related by duality to the weakly-coupled regime based on magnetic 
monopoles. 63,64 This approach also might be relevant in developing a complete, nonperturbative formulation of QED, free of divergences of the charge-charge coupling at small length scales. ${ }^{65}$ A step towards such a theory has been made by showing that the coupling of an electric charge to lines of magnetic flux increases less rapidly, as the length scale decreases, than the coupling of two electric charges, thus reminiscent of the phase of supersymmetric gauge theories corresponding to a weakly-interacting system of magnetic monopoles and abelian photons. ${ }^{66}$

Since the gauge groups of the superstring or heterotic string theory only lead to the inclusion of Chan-Paton factors in the amplitudes, finiteness properties at arbitrary genus and the elimination of large-order divergences continue to hold. While multi-loop modular invariance of type II superstring theory has been formally demonstrated, ${ }^{67}$ consistency of the model requires the vanishing of global gravitational anomalies which could cause the breakdown of modular invariance. A study of the chiral anomaly shows that it depends on the evaluation of a hexagon diagram, which is known to vanish at one loop. ${ }^{68}$ At higher genus, if the chiral anomaly is calculated using a specific choice of basis for the super-Beltrami differentials, the gauge transformation produces total derivative terms with respect to coordinates of the punctures, ${ }^{69}$ so that only the corner of punctured supermoduli space $s \mathcal{M}_{g, 6}$ defined by $\left[s \mathcal{D}_{0,2} \times\right.$ $\left.s \mathcal{D}_{g, 4}\right] \cup\left[s \mathcal{D}_{0,3} \times s \mathcal{D}_{g, 3}\right] \cup\left[s \mathcal{D}_{0,4} \times s \mathcal{D}_{g, 2}\right] \cup\left[s \mathcal{D}_{0,5} \times s \mathcal{D}_{g, 1}\right] \cup\left[s \mathcal{D}_{0,6} \times s \mathcal{D}_{g, 0}\right]$ contributes to the amplitude. However, if this choice of basis is not valid on a global slice of moduli space of punctured super-Riemann surfaces, the integral over the entire supermoduli space would contain an additional set of total derivative terms, ${ }^{70,71}$ including contributions from the other components of the compactification divisor $\bigcup_{i=1}^{g-1}\left[s \mathcal{D}_{i, 2} \times s \mathcal{D}_{g-i, 4}\right] \cup\left[s \mathcal{D}_{i, 3} \times s \mathcal{D}_{g-i, 3}\right] \cup\left[s \mathcal{D}_{i, 4} \times\right.$ $\left.s \mathcal{D}_{g-i, 2}\right] \cup\left[s \mathcal{D}_{i, 5} \times s \mathcal{D}_{g-i, 1}\right] \cup\left[s \mathcal{D}_{i, 6} \times s \mathcal{D}_{g-i, 0}\right]$.

Further evidence for the absence of a global holomorphic slice of supermoduli space is the mixture of NSNS and RR states when curvature is introduced into the background geometry. The supercharges generating the supersymmetry transformations in the soliton geometry satisfy the equation $Q \cdot V_{B}=0$, where $V_{B}$ is the vertex operator representing the curvature of the background. The solution $Q^{\alpha}=Q_{(0)}^{\alpha}+Q_{(1)}^{\alpha}+\ldots$, where $Q_{(0)}=q$ is the flat space-time supercharge, contains an anti-holomorphic contribution in $Q_{(1)} .{ }^{72}$ This suggests that if the target space-time is temporarily curved during the scattering process, the superstring amplitude may involve a mixing of holomorphic and anti-holomorphic parameters.

It is clear that elementary particle masses are best approximated by the massless fundamental modes of the superstring, since they are much smaller than the Planck scale associated with excited higher-energy states. Certain soliton states of both type II superstring theory and heterotic string theory can be organized into supermultiplets and representations of $S L(2 ; \mathbb{Z})$, and they can be described by extreme black hole geometries with quantum numbers appropriate to the supermultiplets. ${ }^{73,74}$ It has been suggested that they might represent stable elementary particles, ${ }^{74}$ since Bogomol'nyi bounds required for stability are satisfied, but this would be valid only for BPS solitons which become massless for special geometries corresponding to particular points in an appropriate space of solutions for the string equations, 
such as the space of Calabi-Yau solutions. ${ }^{75,76,77}$ Scattering of extreme black holes along the lines of classical theory does appear to resemble elementary particle scattering, with an unstable intermediate state having horizon area greater than the sum of the horizon areas of the extreme black holes. ${ }^{78}$.

A preferable approach to the elementary particle spectrum involves the properties of superstring amplitudes with massless external states. The feasibility of the truncation to the massless sector has been made plausible by the connection recently established between type IIA string theory and eleven-dimensional supergravity, where consistent truncation to the $\mathrm{N}=8$ massless supermultiplet has been demonstrated. ${ }^{79,80}$ From S-matrix theory, the total selfenergy graph summed over all orders alters the momentum-space propagator so that there is a non-perturbative shift to the poles in momentum space corresponding to a non-perturbative shift to the mass associated with the field. Similarly, by analyzing the poles of total scattering amplitudes, summed over genus, with massless external superstring states, it may be possible to determine whether there are non-perturbative shifts to small non-zero masses, which then could be interpreted as particle resonances. The locations of these poles in momentum space will depend on the constants appearing in precise exponential estimates of the amplitudes. To determine these constants, bounds on the contributions of the $2^{g}$ sectors of spin structures given earlier must be replaced by exact integrals in the super-Schottky group parameter space. Bounds on the products over conjugacy classes of primitive elements of Schottky groups must then be replaced by the values of these products as functions on the parameter space and the entire fundamental domain of the super-modular group, rather than proper subsets, should be used as the integration region.

\section{Conclusion}

Using the super-Schottky group parameters as coordinates on supermoduli space and restricting the integration region to a single fundamental domain of the super-modular group, it has been demonstrated that the leading-order dependence of the supermoduli space integrals is exponential with respect to the genus. The physical explanation for the removal of the factorial dependence on the genus in superstring amplitudes is based on a connection between infrared and large-order divergences, following from the genus-dependence of the limits for the Schottky group parameters and leading to their simultaneous elimination as a consequence of supersymmetry. The inclusion of effectively closed infinite-genus surfaces in the string path integral leads to a theoretical prediction for the value of the string coupling which closely approximates the value of the gauge coupling constant at grand unified theory scales. Furthermore, finiteness of the scattering amplitudes is independent of the gauge groups of the superstring and heterotic string theory, so that it would be feasible to combine this property these with a unified theory based on lower-dimensional gauge groups. While summability of the loop expansion for superstring interactions implies that the vacuum is perturbatively stable, non-perturbative effects can be included by inserting boundary states representing transitions from one classical background to another local string geometry. 


\section{Acknowledgements.}

I would like to thank Prof. Elmar Schrohe for his hospitality while completing this research at the Institut für Mathematik, Universität Potsdam. Financial support at the University of Sydney and from the Alexander von Humboldt Foundation is gratefully acknowledged. The initial part of this work, described in the paper 'Modular Invariance and the Finiteness of Superstring Theory', hep-th/95030231, was undertaken at the University of Cambridge. Discussions on superstrings with Dr. N. Berkovits, Dr. J. Briginshaw, Prof. M. Green, Prof. C. M. Hull, Prof. E. Martinec, Dr. K. S. Narain, Prof. A. Strominger and Dr. G. Thompson have been helpful in the writing of the manuscript. 


\section{Appendix}

Labelling of even and odd spin structures by their sectors for genus 1, 2 and 3 .

\section{Genus 1}

$\begin{array}{lll} & & \text { Odd } \\ & (-+) & \\ & (--) & \\ \text { RS } & (+-) & (++)\end{array}$

Genus 2

$\begin{array}{lll} & \text { Even } & \text { Odd } \\ \text { NS } & (-+-+) & \\ \text { NS } & (---+) & \\ \text { NS } & (-+--) & \\ \text { NS } & (----) & (+++-) \\ \text { R } & (++++) & (+-++) \\ & (+-+-) & (++-+) \\ S_{3} & (+--+) & (++--) \\ & (+---) & (-+++) \\ S_{4} & (-++-) & (--++)\end{array}$




\section{Genus 3}

Even

NS

$\mathrm{R}$

$S_{4}$

$S_{5}$

$S_{6}$

$S_{7}$

$S_{8}$

$$
\begin{aligned}
& (-+-+-+) \\
& (-+-+--) \\
& (-+---+) \\
& (-+----) \\
& (---+-+) \\
& (---+--) \\
& (-----+) \\
& (------)
\end{aligned}
$$$$
(+++++-)
$$$$
(+-+-+-)
$$$$
(+++-++)
$$$$
(+-++++)
$$$$
(++++-+)
$$$$
(++++--)
$$$$
(+-+--+)
$$$$
(+-+---)
$$$$
(+--++-)
$$$$
(+---+-)
$$$$
(++-+++)
$$$$
(++--++)
$$$$
(-++++-)
$$$$
(--+-+-)
$$$$
(-++-+-)
$$$$
(--++++)
$$$$
(+--+-+)
$$$$
(+--+--)
$$$$
(+----+)
$$$$
(+-----)
$$$$
(-+++--)
$$$$
(--+--+)
$$$$
(--+---)
$$$$
(-+++-+)
$$$$
(-+-++-)
$$$$
(-+--+-)
$$$$
(---++-)
$$$$
(----+-)
$$

\section{Odd}

$$
\begin{aligned}
& (++++++) \\
& (+-+-++) \\
& (+++-+-) \\
& (+-+++-) \\
& (+++--+) \\
& (+++---) \\
& (+-++-+) \\
& (+-++--) \\
& (+--+++) \\
& (+---++) \\
& (++-+++) \\
& (++--+-) \\
& (-+++++) \\
& (--+-++) \\
& (-+++++) \\
& (--++-+) \\
& (+--+--) \\
& (+-----) \\
& (++---+) \\
& (++----) \\
& (--+--+) \\
& (-+++--) \\
& (--++--) \\
& (--++-+) \\
& (-+-+++) \\
& (-+--++) \\
& (---+++) \\
& (----++)
\end{aligned}
$$


It is well known that modular transformations on the torus transform the even spin structures $(+-),(-+)$ and $(--)$ into each other while leaving invariant the odd spin structure $(++)$. At higher genus, there is a class of modular transformations that are products of genus-1 transformations, each acting on different handles. Denoting the modular transformations by $\rho_{r}$, they map the Ramond structure $R$, or $S_{2}$ in the terminology of $\S 3$, to the sectors $S_{r}^{\prime}$, which consist of $2^{g}$ spin structures. Using only those modular transformations which do not map a Ramond spin structure into a Neveu-Schwarz spin structure, the $N S, R$ and $S_{r}^{\prime}$ are given for genus 2 and 3 .

\section{Genus 2}

$$
\begin{aligned}
& \mathrm{NS} \\
& (-+-+) \\
& (---+) \\
& (----) \\
& (-+--) \\
& S_{4}^{\prime} \\
& (+++-) \\
& (-++-) \\
& (-+++) \\
& (++++)
\end{aligned}
$$

$$
\begin{aligned}
& \mathrm{R} \\
& (+++-) \\
& (+-+-) \\
& (+-++) \\
& (++++) \\
& S_{5}^{\prime} \\
& (++--) \\
& (+---) \\
& (+-++) \\
& (++++)
\end{aligned}
$$

$S_{3}^{\prime}$

$(++-+)$

$(+--+)$

$(+-++)$

$(++++)$

$S_{6}^{\prime}$

$(+++-)$

$(--+-)$

$(--++)$

$(++++)$

\section{Genus 3}

NS

$$
\begin{aligned}
& (-+-+-+) \\
& (-+-+--) \\
& (-+---+) \\
& (-+----) \\
& (---+-+) \\
& (---+--) \\
& (-----+) \\
& (------)
\end{aligned}
$$

R

$$
\begin{aligned}
& (+++++-) \\
& (+++-+-) \\
& (+-+++-) \\
& (+-+-+-) \\
& (++++++) \\
& (+++-++) \\
& (+-++++) \\
& (+-+-++)
\end{aligned}
$$

$S_{3}^{\prime}$

$$
\begin{gathered}
(++++-+) \\
(+++--+) \\
(+-++-+) \\
(+-+--+) \\
(++++++) \\
(+++-++) \\
(+-++++) \\
(+-+-++)
\end{gathered}
$$




\section{$S_{4}^{\prime}$}

$$
\begin{aligned}
& (++++--) \\
& (++-+--) \\
& (+-++--) \\
& (+-+---) \\
& (++++++) \\
& (+++-++) \\
& (+-++++) \\
& (+-+-++) \\
& S_{7}^{\prime}
\end{aligned}
$$$$
(+++++-)
$$$$
(+++-+-)
$$$$
(-++++-)
$$$$
(--+-+-)
$$$$
(++++++)
$$$$
(+++-++)
$$$$
(-+++++)
$$$$
(-++-++)
$$$$
S_{10}^{\prime}
$$$$
(++++--)
$$$$
(++-+--)
$$$$
(+-++--)
$$$$
(+--+--)
$$$$
(++++++)
$$$$
(++-+++)
$$$$
(+-++++)
$$$$
(+--+++)
$$$$
S_{13}^{\prime}
$$$$
(++++-+)
$$$$
(+++--+)
$$$$
(-+++-+)
$$$$
(-++--+)
$$$$
(++++++)
$$$$
(+++-++)
$$$$
(-+++++)
$$$$
(-++-++)
$$

$S_{5}^{\prime}$

$$
\begin{aligned}
& (+++++-) \\
& (++-++-) \\
& (+-+++-) \\
& (+--++-) \\
& (+--++-) \\
& (++++++) \\
& (++-+++) \\
& (+--+++)
\end{aligned}
$$$$
S_{8}^{\prime}
$$$$
(+++++-)
$$$$
(+++-+-)
$$$$
(--+++-)
$$$$
(--+-+-)
$$$$
(++++++)
$$$$
(+++-++)
$$$$
(--++++)
$$$$
(--+-++)
$$$$
S_{11}^{\prime}
$$$$
(++++-+)
$$$$
(++---+)
$$$$
(+-++-+)
$$$$
(+----+)
$$$$
(++++++)
$$$$
(++--++)
$$$$
(+-++++)
$$$$
(+---++)
$$$$
S_{14}^{\prime}
$$$$
(++++--)
$$$$
(+++---)
$$$$
(-+++--)
$$$$
(-++---)
$$$$
(++++++)
$$$$
(+++-++)
$$$$
(-+++++)
$$$$
(-++-++)
$$

$S_{6}^{\prime}$

$(+++++-)$
$(++--+-)$
$(+-+++-)$
$(+---+-)$
$(++++++)$
$(++--++)$
$(+-++++)$
$(+---++)$

\section{$S_{9}^{\prime}$}

$(++++-+)$

$(++-+-+)$

$(+-++-+)$

$(+--+-+)$

$(++++++)$

$(++-+++)$

$(+-++++)$

$(+--+++)$

$S_{12}^{\prime}$

$(++++--)$

$(++----)$

$(+-+---)$

$(+-----)$

$(++++++)$

$(++--++)$

$(+-++++)$

$(+---++)$

$S_{15}^{\prime}$

$(++++-+)$

$(+++---)$

$(-+++--)$

$(-++---)$

$(++++++)$

$(+++-++)$

$(-+++++)$

$(-++-++)$ 


$$
\begin{aligned}
& S_{16} \\
& (++++--) \\
& (+++---) \\
& (--++--) \\
& (--+---) \\
& (++++++) \\
& (+++-++) \\
& (--++++) \\
& (--+-++) \\
& S_{19}^{\prime} \\
& (+++++-) \\
& (++-++-) \\
& (--+++-) \\
& (--+-+-) \\
& (++++++) \\
& (++-+++) \\
& (--++++) \\
& (---+++)
\end{aligned}
$$

$$
\begin{aligned}
& S_{17}^{\prime} \\
& (+++++-) \\
& (++-++-) \\
& (-++++-) \\
& (-++--+) \\
& (++++++) \\
& (++-+++) \\
& (-+++++) \\
& (-+-+++) \\
& S_{20}^{\prime} \\
& (+++++-) \\
& (++--+-) \\
& (--+++-) \\
& (----+-) \\
& (++++++) \\
& (++--++) \\
& (--++++) \\
& (----++)
\end{aligned}
$$$$
S_{18}^{\prime}
$$$$
(+++++-)
$$$$
(++--+-)
$$$$
(-++++-)
$$$$
(-+--+-)
$$$$
(++++++)
$$$$
(++--++)
$$$$
(-+++++)
$$$$
(-+--++)
$$

It can be verified that the spin structures match so that the tachyon divergence associated with the $\left|K_{n}\right| \rightarrow 0$ limit is cancelled for each of the sectors $S_{r}^{\prime}$ at genus 2 and 3 . In $\S 4$, this property is shown to hold for all genus. 


\section{REFERENCES}

1. S. Davis, Class. Quantum Grav. 11 (1994) 1185

2. S. Davis, J. Math. Phys. $\underline{36}(2)$ (1995) 648

3. H. Aoyama, H. Kikuchi, I. Okouchi, M. Sato and S. Wada, Nucl. Phys. B5553 (1999) 664

4. M. Salmhofer and C. Wieczerkowski, 'Positivity and convergence in fermionic quantum field theory' (1999) math-ph/9909002

5. J. Polchinski, Phys. Rev. D50 (1994) 6041

6. E. D’Hoker and D. H. Phong, Rev. Mod. Phys. $\underline{61}$ (1988) 917

7. N. Berkovits, Nucl. Phys. $\underline{B 408}$ (1993) 43

8. J. L. Petersen, J. R. Sidenius and A. K. Tollsten, Nucl. Phys. B317 (1989) 109

9. B. E. W. Nilsson, A. K. Tollsten and A. Watterstam, Phys. Lett. B222 (1989) 399

10. G. Cristofano, M. Fabbrichesi and K. Roland, Phys, Lett. B236 (1990) 159

11. P. DiVecchia, K. Hornfeck, M. Frau, A. Lerda and S. Sciuto, Phys. Lett. $\underline{B 211}$ (1988) 301

12. J. L. Petersen, J. R. Sidenius and A. K. Tollsten, Phys. Lett. B213 (1988) 30

13. A. Bellini, G. Cristofano, M. Fabbrichesi and K. Roland, Nucl. Phys. $\underline{B 356}$ (1991) 69

14. S. Davis, Class. Quantum Grav. $\underline{7}$ (1990) 1887

15. G. S. Danilov, Phys. Atom. Nucl. $\underline{60}$ (1997) 1495

16. G. S. Danilov, Nucl. Phys. $\underline{B 463}$ (1996) 443

17. G. E. Andrews, The Theory of Partitions, Encyclopedia of Mathematics and Its Applications, Vol. 2, ed. by G.-C. Rota, Addison-Wesley, Reading 1976)

18. Z. Star, Aequationes Math. $\underline{13}$ (1975) 279

19. S. Davis, Phys. Lett. $\underline{432 B}$ (1998) 317

20. L. Crane and J. Rabin, Commun. Math. Phys. 113 (1988) 601

21. S. Davis, Classical and Quantum Gravity $\underline{6}$ (1989) 1791; Mod. Phys. Lett. $\underline{A 9}$ (1994) 1299

22. S. B. Giddings, E. Martinec and E. Witten, Phys. Lett. $\underline{B 176}$ (1986) 362

23. J. J. Atick, G. Moore and A. Sen, Nucl. Phys. B308 (1988) 1

24. N. Berkovits, Nucl. Phys. B304 (1988) 537

25. N. Berkovits, Nucl. Phys. B395 (1993) 77 
26. G. Cristofano, R. Musto, F. Nicodemi and R. Pettorino, Phys. Lett. $\underline{B 217}$ (1989) 65

27. D. Lust and S. Theisen, Lectures on String Theory (Berlin: Springer-Verlag, 1989)

28. S. Mandelstam, 'The Interacting String Picture and Functional Integration', Unified String Theories, ed. by M. Green and D. Gross World Scientific, Singapore 1986

29. U. Carow-Watamura, Z. F. Ezawa and S. Watamura, Nucl. Phys. B315 (1989) 166

30. R. C. Gunning, Lectures on Riemann Surfaces Princeton University Press, Princeton 1966

31. K. Nishimura, Prog. Theor. Phys. $\underline{84}$ (1990) 360

32. D. Mumford, Tata Lectures on Theta Functions Birkhäuser, Basel 1982

33. M. Kaku, Introduction to Superstrings Springer-Verlag, Berlin 1988

34. E. D'Hoker and D. H. Phong, 'Two-Loop Superstrings IV. The Cosmological Constant and Modular Forms', UCLA/01/TEP/27, Columbia/MATH/01, hep-th/0111040

35. E. D'Hoker and D. H. Phong, 'Two-Loop Superstrings II. The Chiral Measure on Moduli Space', UCLA/01/TEP/25, Columbia/MATH/01, hep-th/0110283

36. G. S. Danilov, 'Explicit Calculation of Multiloop Amplitudes in the Superstring Theory' St. Petersburg preprint, PNPI-1872, hep-th/9305029

37. G. S. Danilov, Phys. Rev. D51 (1995) 4539

38. D. Friedan and S. Shenker, Phys. Lett. $\underline{B 175}$ (1986) 287

D. Friedan and S. Shenker, Nucl. Phys. $\underline{B 281}$ (1987) 509

39. E. Martinec, Nucl. Phys. $\underline{B 281}$ (1987) 157

40. D. Friedan, E. Martinec and S. Shenker, Nucl. Phys. $\underline{B 198}$ (1982) 93

41. N. Berkovits, private communication

42. O. Lechtenfeld and A. Parkes, Nucl. Phys. B332 (1990) 39

43. I. Antoniadis, E. Gava, K. S. Narain and T. R. Taylor, Nucl. Phys. B4113 (1994) 162

44. M. Bershdasky, S. Cecotti, H. Ooguri and C. Vafa, Commun. Math. Phys. 165 (1994) 311

45. S. Hamidi and C. Vafa, Nucl. Phys. $\underline{B 279}$ (1987) 465

46. H. Ooguri and C. Vafa, Nucl. Phys. $\underline{B 451}$ (1995) 121

47. N. Berkovits and C. Vafa, Nucl. Phys. B B433 (1995) 123

48. B. Zwiebach, Nucl. Phys. B390 (1993) 33 
49. H. Ooguri and N. Sakai, Nucl. Phys. B312 (1988) 435

50. J. J. Atick, J. M. Rabin and A. Sen, Nucl. Phys. B299 (1988) 299

51. S. Mandelstam, Phys. Lett. $\underline{B 277}$ (1992) 82

52. J. D. Cohn, Nucl. Phys. B306 (1988) 239

53. B. de Carlos, J. A. Casas and C. Munoz, Nucl. Phys. B399 (1993) 623

54. B. Lautrup, Phys. Lett. $\underline{69 B}(1995) 109$

P. Oleson, Phys. Lett. $\underline{73 B}(1978) 327$

55. M. Gutperle, Nucl. Phys. B444 (1995) 123

56. M. B. Green, Phys. Lett. B354 (1995) 271

57. M. J. Duff, G. W. Gibbons and P. K. Townsend, Phys. Lett. B332 (1994) 321

58. J. Polchinski, Phys. Rev. Lett. $\underline{75}$ (1995) 4274

59. M. B. Green, Phys. Lett. $\underline{B 329}$ (1994) 435

60. G. W. Gibbons, 'Aspects of supergravity' in Supersymmetry, Supergravity and Related Topics, ed. by F. Dell Aguilla, J. A. Azcarraga and L. E. Ibanez, Proceedings of the Fifteenth G.I.F.T. Seminar on Theoretical Physics, World Sceintific Publishing, Singapore 1984

61. S. Davis, 'Supersymmetry in Anti-De Sitter Space', Ph. D. Thesis, University of Cambridge (1985)

62. E. Caianello, Nuovo Cimento, $\underline{3}$ (1956) 223

63. N. Seiberg and E. Witten, Nucl. Phys. B426 19 (1994); Nucl. Phys. B431 (1994) 484

64. C. Vafa and E. Witten, Nucl. Phys. $\underline{B 431}$ (1994) 3

65. C. M. Hull and P. K. Townsend, Nucl. Phys. B438 (1995) 109

66. M. J. Duff, Nucl. Phys. B335 (1990) 610

67. O. Yasuda, Nucl. Phys. B318 (1989) 397

68. L. Alvarez-Gaume and E. Witten, Nucl. Phys. B234 (1984) 269

69. O. Yasuda, Phys. Lett. $\underline{215 B}$ (1988) 306

70. G. Falqui and C. Reina, Commun. Math. Phys. 128 (1990) 247

71. J. A. Dominguez Pérez, D. Hernández Ruipérez and C. Sancho de Salas, J. Geom. Phys. $\underline{21}$ (1997) 199

72. D. Berenstein and R. G. Leigh, Phys. Rev. $\underline{D 60}$ (1999) 106002 
73. M. J. Duff and J. Rahmfeld, Phys. Lett. $\underline{B 345}$ (1995) 441

74. M. J. Duff, R. R. Khuri, R. Minasian and J. Rahmfeld, Nucl. Phys. B418 (1994) 195

75. B. de Wit and A. van Proeyen, Commun. Math. Phys. $\underline{149}$ (1992) 307

76. B. R. Greene, D. R. Morrison and A. Strominger, Nucl. Phys. B451 (1995) 109

77. M. Cvetic and D. Youm, Phys. Lett. $\underline{359 B}$ (1995) 87

78. S. W. Hawking, 'The Event Horizon', Black Holes : Les Houches 1972, ed. by C. De Witt and B. S. De Witt, Gordon and Breach, New York, 1973

79. M. Duff and C. Pope, Nucl. Phys. B255 (1985) 355

80. B. de Wit and H. Nicolai, Nucl. Phys. $\underline{B 255}$ (1985) 211 